\title{
Charting the coming synergy between lattice QCD and high-energy phenomenology
}

\author{
T. J. Hobbs $\odot,{ }^{1,2, *}$ Bo-Ting Wang, ${ }^{1, \dagger}$ Pavel M. Nadolsky, ${ }^{1, \star}$ and Fredrick I. Olness ${ }^{1, \S}$ \\ ${ }^{1}$ Department of Physics, Southern Methodist University, Dallas, Texas 75275-0175, USA \\ ${ }^{2}$ Jefferson Lab, EIC Center, Newport News, Virginia 23606, USA
}

(Received 23 April 2019; published 27 November 2019)

\begin{abstract}
Building upon the PDFSense framework developed in Wang et al. [Phys. Rev. D 98, 094030 (2018)], we perform a comprehensive analysis of the sensitivity of present and future high-energy data to a number of quantities commonly evaluated in lattice gauge theory, with a particular focus on the integrated Mellin moments of nucleon parton distribution functions, such as $\langle x\rangle_{u^{+}-d^{+}}$and $\langle x\rangle_{g}$, as well as $x$-dependent quark quasidistributions - in particular, that of the isovector combination. Our results demonstrate the potential for lattice calculations and phenomenological quark distributions informed by high-energy experimental data to cooperatively improve the picture of the nucleon's collinear structure. This will increasingly be the case as computational resources for lattice calculations further expand, and QCD global analyses continue to grow in sophistication. Our sensitivity analysis suggests that a future lepton-hadron collider would be especially instrumental in providing phenomenological constraints to lattice observables.
\end{abstract}

DOI: 10.1103/PhysRevD.100.094040

\section{INTRODUCTION}

Owing to steady theoretical progress and the growing availability of computational resources, the ability of perturbative QCD (pQCD) to predict parton-level processes at high energies has continued to improve in recent years, with accuracies now reaching next-to-next-to-leading order (NNLO) in many circumstances. Inevitably, however, predictions for experiments involving hadronic collisions require precise knowledge of the structure of initial-state hadrons at comparatively small energy scales similar to the nucleon mass, $\Lambda \sim M$, at which $\alpha_{s}(\Lambda) \sim 1$ is too large to permit a converging diagrammatic expansion of the relevant amplitudes. This general consequence of the negative $\beta$-function of QCD is realized in the theory of spinaveraged deeply inelastic lepton-nucleon scattering, for example, in the factorization of physical cross sections into perturbatively calculable short-distance matrix elements and inherently nonperturbative long-distance parton distribution functions (PDFs), $q(x, \mu)$, of the quark-to-hadron light-front momentum fraction $x=k^{+} / p^{+}$and factorization scale $\mu$.

\footnotetext{
*tjhobbs@smu.edu

†botingw@mail.smu.edu

*nadolsky@physics.smu.edu

§olness@smu.edu
}

Published by the American Physical Society under the terms of the Creative Commons Attribution 4.0 International license. Further distribution of this work must maintain attribution to the author(s) and the published article's title, journal citation, and DOI. Funded by SCOAP ${ }^{3}$.
Given the nonperturbative nature of the collinear PDFs, the prevailing recourse has traditionally been either to fit them in comprehensive analyses of global data using flexible parametric forms [1-5] or to calculate them in the context of models or effective theories [6-15] that aim to capture specific aspects of QCD-e.g., its pattern of dynamical chiral symmetry breaking. Determination of precise effective parametrizations of the collinear PDFs in the $\overline{\mathrm{MS}}$ factorization scheme from the global QCD analysis of experimental measurements has grown into a multifaceted research field-for reviews of its status see, e.g., Refs. [16,17]. Parallel to these efforts, the past couple of decades have seen a complementary effort founded in the use of lattice gauge theory techniques to either indirectly compute the $x$ dependence of the PDFs themselves or, at minimum, determine the integrated moments of the parton distributions in Mellin space. (For a comprehensive review, we refer the reader to the recent white paper, Ref. [18].)

By definition, the PDFs are intrinsically nonlocal correlation functions constructed between parton fields with lightlike spacetime separation along the $\xi^{-}$direction at fixed light-front time $\xi^{+}=0$ : viz., $\sim\left\langle p\left|\bar{q}\left(\xi^{-}\right) \hat{\mathcal{O}} q(0)\right| p\right\rangle$. Dynamically simulating such matrix elements on a hypercubic lattice, however, is numerically problematic, given the fact ${ }^{1}$ that $x^{2}=x^{+} x^{-}-x_{\perp}^{2}=0$ can only trivially hold at the origin in a Euclidean spacetime, for which

\footnotetext{
${ }^{1}$ In defining light-front variables, we assume a convention in which the components of an arbitrary 4-vector $a^{\mu}$ transform as $a^{ \pm}=a^{0} \pm a^{3}$.
} 
$x_{\mathrm{E}}^{2}=x_{1}^{2}+\cdots+x_{4}^{2}$. In contrast, the integrated Mellin moments of the quark distributions have a direct interpretation in terms of the matrix elements of local operators and can be accessed on a Euclidean lattice via an operator product expansion (OPE). Moments computed in this fashion are informative in the sense that they encapsulate aspects of the nonperturbative dynamics responsible for a hadron's low-energy structure-for instance, the magnitude of the nucleon's collinear magnitude carried by its total $u$-quark content,

$$
\langle x\rangle_{u^{+}}=\int_{0}^{1} d x x[u+\bar{u}]\left(x, \mu_{F}\right) .
$$

Lattice calculations generally evaluate moments such as Eq. (1) using a scheme and renormalization scale $\mu^{\text {lat }}$ chosen to match the $\overline{\mathrm{MS}}$ scheme usually employed by phenomenologists. Most often in the literature, this scale is taken to be $\mu^{\text {lat }}=2 \mathrm{GeV}$, and in this analysis we shall for consistency compute moments at a matching factorization scale, $\mu_{F}=\mu^{\text {lat }}=2 \mathrm{GeV}$, unless otherwise indicated. Various attempts have been made to determine the $x$ dependence of the PDFs by computing a sufficient number of moments in Mellin space that the transform into PDF space can be determined (typically with the help of some parametrization). In practice, however, the mixing among operators of successively higher spin and the resulting signal-to-noise issues become less controlled as additional covariant derivatives are inserted to obtain PDF moments of higher order. In effect, only a small number of moments can be accessed on the lattice-presently, up to the quark distributions' third moment, $\left\langle x^{3}\right\rangle_{q^{+}}$(although there are recent suggestions that perhaps several more may become available in the near future). It should be noted that the uncertainties of the lattice moments typically grow with increasing order.

Still, the ostensible ability of lattice gauge theory to access even several moments of the PDFs has long presented the possibility of determining (or at least constraining) the parton distributions directly from a firstprinciples QCD calculation. Indeed, with a sufficiently restrictive parametric form for the quark distribution of a given flavor, the latter can be fully determined given enough moments [19]; for example, if the PDF of the $u$-quark distribution is taken to have a very simple $x$ dependence given by $u\left(x, Q_{0}\right)=\alpha x^{\beta}(1-x)^{\gamma}$, knowledge of three distinct moments would in principle be adequate to parametrically determine (up to some uncertainty) the above-noted distribution. At the same time, however, both the diversity of the experimental data inputs and the sophistication of modern QCD analyses are such that much more flexible parametric forms are required, and lattice calculations remain far below the requisite level of precision across the many flavors and moment orders needed to be competitive in a complete determination of the PDFs according to such a procedure.
More recently, a promising method which may allow the calculation of the PDFs' $x$ dependence on the QCD lattice in terms of parton quasidistribution functions (qPDFs) has been introduced by Ji [20], as well as the more recent pseudo-PDF concept first developed in Ref. [21] of Radyushkin. Extracting information from quasidistributions requires an accompanying large momentum effective theory (LaMET) for performing the necessary ultraviolet matchings that are realized as convolutional relations of the form

$\tilde{q}\left(x, P_{z}, \tilde{\mu}\right)=\int d y Z\left(\frac{x}{y}, \frac{\Lambda}{P_{z}}, \frac{\mu}{P_{z}}\right) q(y, \mu)+\mathcal{O}\left(\frac{\Lambda^{2}}{P_{z}^{2}}, \frac{M^{2}}{P_{z}^{2}}\right)$,

which relate the quasidistribution $\tilde{q}$ to the traditional phenomenological PDF $q$ with the usual support over $x \in[0,1] .^{2}$ This matching depends critically upon the pQCD-calculable ultraviolet matching function, $Z$. In practice, the quasidistribution $\tilde{q}\left(x, P_{z}, \tilde{\mu}\right)$ [i.e., the left-hand side (LHS) of Eq. (2)] may be evaluated on the lattice for a specific choice of the longitudinal hadron momentum $P_{z}$ and the usual PDF extracted by numerical inversion of Eq. (2). This method therefore has the potential to yield information on the $x$ dependence of the PDFs themselves, up to knowledge of dynamical and mass-dependent corrections, the perturbative order of the matching kernel $Z$, and technical details of the actual lattice calculation-for instance, artifacts arising from the finite lattice spacing or signal-to-noise problems. In addition, it should be pointed out that limitations to this procedure remain, especially given the fact that the lattice calculations and LaMET procedure are in a relatively early stage of theoretical development—much as there are limitations to the lattice computed PDF moments as well.

For the reasons noted above, as computational resources continue to grow, it will be necessary to reconcile the output of lattice-based methods (especially, concerning the PDF moments and quasidistributions) with work in the context of QCD global analyses. This will necessarily go both directions: benchmarking the lattice calculations with knowledge of the PDFs from phenomenological analyses, and constraining QCD analyses with the output of the lattice. Laying the groundwork for this synergy will require a comprehensive understanding of the relation between

\footnotetext{
${ }^{2}$ Traditional PDFs are correlations at a fixed light-front time $\xi^{+}=0$ and functions of the parton-to-hadron momentum fraction $x=k^{+} / P^{+}$. The qPDFs, on the other hand, are analogous fractions of longitudinal momentum $x=k_{z} / P_{z}$, which can be matched in the context of LaMET via Eq. (2). In the subsequent plots mapping the sensitivity of CTEQ-TEA data to qPDFs, we compute the qPDF at a specific $x=k_{z} / P_{z}$ by means of Eq. (2), with the sensitivity computed on the basis of the variation of the underlying PDF appearing inside the convolution. For this reason, sensitivity plots are always shown as maps giving the pull of data at a given, matched $x_{i} \in[0,1]$ and $\mu_{i}$ to the qPDF at a specific $x=k_{z} / P_{z} \in(-\infty, \infty)$.
} 
phenomenological constraints placed on the PDFs determined in fits (and, by extension, the $x$-weighted moments computed therefrom) and information obtained from the lattice.

In this analysis we systematically canvass these issues, using the recently developed PDFSense framework of Ref. [22] to present a comprehensive summary of the differential impact modern data have upon knowledge of the PDF Mellin moments evaluated from phenomenological fits-as well as which data give the leading contributions to present understanding of one of the typical quasidistributions, that of the isovector combination, $u-d$. The remainder of the paper is therefore as follows.

After a brief review of the formalism and a description of the PDFSense methodology, in Sec. II we review the constraints HEP data place on the lowest moments for several light-quark $q^{ \pm}$distributions and the gluon. We also discuss the degree of the agreement among the main experiments that constrain the relevant PDF flavors at $x>0.1$. For this purpose, we introduce a new statistical indicator, $L_{2}$ sensitivity, which is especially convenient for exploring the aggregate constraints of the individual experiments on the PDFs, and the pulls they exert on the PDFs in various momentum fraction intervals.

In Sec. III, we illustrate the constraints that the data place on the $u-d$ quasidistributions by computing them from the CT14HERA2 NNLO PDFs [5] at several choices of the momentum fraction, $\pm x$, and hadronic boost momentum, $P_{z}$, using Eq. (2). Section IV demonstrates the sizable potential impact future measurements at a high-luminosity lepton-hadron collider will have on these quantities by analyzing pseudodata generated from a combination of Monte Carlo generated information with cross section predictions based upon CT14HERA2 NNLO. Section V contains a number of conclusions drawn from our analysis of the PDF moments and qPDFs regarding expected consequences of implementing lattice information into future global fits. In Sec. VI we provide a number of closing observations, focusing on points that will allow this work to be leveraged in the future. Last, Appendix collects several tables - counterparts to Figs. 13 and 14-summarizing the aggregated impact on lattice QCD observables of the HEP experiments considered in this work.

\section{THE SENSITIVITY OF HEP DATA TO PDF MELLIN MOMENTS}

\section{A. Theory of PDF Mellin moments}

The $x$-weighted moments of the PDFs have long been of interest to practitioners of QCD analyses on the logic that they may provide the necessary input to either reconstruct or at least constrain the PDFs determined in global fits. The accessibility of these moments to lattice gauge techniques is facilitated by the OPE [23-28], which allows an expansion of the PDF moments in terms of matrix elements of well-defined, local twist-2 operators which can be evaluated in a discretized Euclidean spacetime. Subsequently, the Mellin moments themselves may be derived via algebraic relations from the matrix elements of these twist-2 operators. In principle, it is possible to reconstruct a given PDF's $x$ dependence via an inverse Mellin transform if its moments $\left\langle x^{n}\right\rangle_{q}$ are known to all orders, as noted in Sec. I.

The crucial relation that connects $x$-dependent parton distributions $q(x)$ to an $n$-dependent tower of integrals in Mellin space is the inverse Mellin transform, which enables one in principle to reconstruct integrated Mellin moments of the PDFs. These PDF Mellin moments have the general form

$$
\left\langle x^{n}\right\rangle_{q}=\int_{0}^{1} d x x^{n}\left[q(x)+(-1)^{n+1} \bar{q}(x)\right] .
$$

Using Eq. (3), it is possible to define a collection of PDF moments $\left\langle x^{n}\right\rangle_{q^{ \pm}}$which are actually calculable on the QCD lattice, such as $\langle x\rangle_{u}^{+}$of Eq. (1). These are

$$
\begin{gathered}
\left\langle x^{n}\right\rangle_{q^{+}}=\left\langle x^{n}\right\rangle_{q} \text { for } n=2 \ell-1, \\
\left\langle x^{n}\right\rangle_{q^{-}}=\left\langle x^{n}\right\rangle_{q} \text { for } n=2 \ell,
\end{gathered}
$$

where $\ell \in \mathbb{Z}^{+}$such that the lattice may provide, for instance, $\langle x\rangle_{u^{+}},\left\langle x^{2}\right\rangle_{u^{-}},\left\langle x^{3}\right\rangle_{u^{+}}$. Moreover, the PDFs themselves can be unfolded from a complete set of Mellin moments via the inverse Mellin transform,

$$
q(x)+(-1)^{n+1} \bar{q}(x)=\frac{1}{2 \pi i} \int_{c-i \infty}^{c+i \infty} d n x^{-n-1}\left\langle x^{n}\right\rangle_{q} .
$$

In practice, however, lattice QCD techniques currently enable the calculation of the few lowest PDF moments. Once the accuracy of these computations improves, theoretical constraints in the form of lattice-calculated PDF moments (or $x$-dependent information unfolded from parton quasidistributions, discussed in Sec. III) might eventually be implemented as $\chi^{2}$ penalties in global QCD analysesessentially, taking lattice data as theoretical priors to constrain the likelihood function of a global fit. For example, exploratory studies based on Bayesian profiling have suggested that lattice calculations even with somewhat sizable uncertainties can still provide powerful constraints to PDFs in regions that are relatively unconstrained by experimental data (see Ref. [18] and references therein). In the remainder of this subsection, we review the essential theory for accessing the integrated moments of the PDFs, with a special focus on unpolarized distributions, given their importance to high-energy phenomenology. (Although it is worth noting that, given the comparative paucity of spin-dependent data, it is reasonable to expect that lattice calculations for the moments of helicity distributions may more quickly become competitive against fitted 
spin-dependent PDFs; we defer such considerations, however, to future work.)

The OPE expands hadronic matrix elements of nonlocal products of field operators in terms of local operator matrix elements weighted by Wilson coefficients that obey renormalization group evolution [24,29]. It is then possible to calculate these coefficients in the context of QCD perturbatively (i.e., they embody the relevant short-distance dynamics), while the local operator matrix elements are nonperturbative (depending on the details of the longdistance physics). In the case relevant for the present study, the matrix elements of twist- 2 operators may be expanded by the OPE as

$\frac{1}{2} \sum_{s}\left\langle p, s\left|\mathcal{O}_{\left\{\mu_{1}, \ldots, \mu_{n+1}\right\}}^{q}\right| p, s\right\rangle=2 v_{q}^{n+1}\left[p_{\mu_{1}} \cdots p_{\mu_{n+1}}-\right.$ traces $]$

$\left\langle p, s\left|\mathcal{O}_{\left\{\sigma \mu_{1}, \ldots, \mu_{n+1}\right\}}^{5 q}\right| p, s\right\rangle=\frac{1}{n+2} a_{q}^{n+1}\left[s_{\sigma} p_{\mu_{1}} \cdots p_{\mu_{n+1}}-\right.$ traces $]$,

where $p$ and $s$ represent the nucleon 4-momentum and spin, respectively, $q$ indicates the flavor of the relevant quark field, and \{\} stands for index symmetrization. Higher-twist terms enter as power suppressed corrections in $1 / Q^{2}$ [30], so here we consider only the contribution of Mellin moments from twist-2 operators. For the quark fields, the twist-2 operators occurring in the OPE expressions above are constructed from the usual bilinears as

$$
\mathcal{O}_{\left\{\mu_{1}, \ldots, \mu_{n+1}\right\}}^{q}=\left(\frac{i}{2}\right)^{n} \bar{q}(x) \gamma_{\left\{\mu_{1}\right.} \stackrel{\leftrightarrow}{D}_{\mu_{2}} \cdots \stackrel{\leftrightarrow}{D}_{\left.\mu_{n+1}\right\}} q(x)
$$

and

$\mathcal{O}_{\left\{\sigma \mu_{1}, \ldots, \mu_{n+1}\right\}}^{5 q}=\left(\frac{i}{2}\right)^{n+1} \bar{q}(x) \gamma_{\{\sigma} \gamma_{5} \stackrel{\leftrightarrow}{D}_{\mu_{1}} \cdots \stackrel{\leftrightarrow}{D}_{\left.\mu_{n+1}\right\}} q(x)$

where

$\stackrel{\leftrightarrow}{\mathcal{D}}_{\mu}=\frac{1}{2}\left(\overrightarrow{\mathcal{D}}_{\mu}-\overleftarrow{\mathcal{D}}_{\mu}\right), \quad \overrightarrow{\mathcal{D}}_{\mu}=\vec{\partial}_{\mu}-i g t_{a} A_{\mu}^{a}(z)$,

$\overleftarrow{\mathcal{D}}_{\mu}=\overleftarrow{\partial}_{\mu}+i g t_{a} A_{\mu}^{a}(z)$

are the gauge covariant derivatives, $A_{\mu}^{a}(z)$ denotes gluon fields, and $t_{a}$ represents the eight standard generators of the $\mathrm{SU}(3)$ color group. We note that $\{\cdots\}$ in the expressions above denotes symmetrization over the enclosed indices. In Eqs. (6) and (7), $v_{q}^{n+1}$ and $a_{q}^{n}$ are identifiable with the $n$ thorder moments of the twist-2 PDFs of unpolarized and longitudinally polarized nucleons, respectively $[18,31]$ :

$$
\begin{aligned}
& v_{q}^{n+1}=\int_{0}^{1} d x x^{n} q(x, \mu), \\
& a_{q}^{n}=\int_{0}^{1} d x x^{n} \Delta q(x, \mu) .
\end{aligned}
$$

Last, we note that analogous matrix elements are responsible for moments of the gluon distribution, with the lowest latticeaccessible moment $\langle x\rangle_{g}$ for the total gluon momentum fraction given by the insertion of a twist-2 operator constructed from the gluon field strength as $\mathcal{O}_{\mu_{1} \mu_{2}}^{g}=-\operatorname{Tr}\left(\mathcal{G}_{\mu_{1} \alpha} \mathcal{G}_{\mu_{2} \alpha}\right)$.

Insofar as the LHS expressions of Eqs. (6) and (7) can be formulated in terms of lattice gauge theory and evaluated on a discretized Euclidean spacetime, the PDF moments in Eq. (11) are themselves directly accessible on the QCD lattice through the direct evaluation of nucleonic matrix elements of twist- 2 operators. For reasons that we sketch below, however, the extraction of higher moments is complicated by operator-mixing effects, and modern lattice calculations can reliably extract only the lowest few moments in practice $[19,32,33]$. Various systematic errors in generic lattice calculations are reviewed and assessed in Ref. [34], with the dominant systematic errors in evaluations of Mellin moments arising from power-divergent operator mixing and renormalization effects. Powerdivergent mixing is associated with an $O(4)$ symmetry breaking inherent to the Euclidean spacetime discretization of lattice calculations: the lattice regulator breaks Lorentz symmetry, causing radiative divergences in operators of different mass dimensions to mix together [35]. The renormalization of nonlocal operators on a discretized lattice induces another error: the renormalized fields that are nonperturbatively determined on the lattice are power divergent. In addition to these, a number of other systematic effects generally enter lattice QCD calculations, including corrections from the unphysically large quark (or pion) masses often used as well as the associated chiral extrapolations to the physical pion mass. Moreover, details involved in the extraction of lattice signals as a function of lattice time contribute to the landscape of systematic uncertainties. The effort to control these and other lattice artifacts partially depends upon the ability of lattice practitioners to benchmark their calculations against phenomenological knowledge. Exploring the capacity of high-energy data to tighten these benchmarks is a primary motivation for the present study.

As for the present status of lattice QCD calculations for some of the lowest PDF moments, we present in Table I a numerical comparison of several recent lattice extractions (rightmost column) with the results of computing the same moments from CT14HERA2 NNLO [5] (middle column). Notably, despite significant improvements in recent years, the uncertainties of modern lattice QCD calculations remain considerably larger than the analogous errors for the moments computed from fitted PDFs, which we quote 
TABLE I. The most recent PDF moment results obtained from lattice QCD calculations (central column) compared to the analogous results determined from fitted PDFs, here, CT14HERA2 NNLO (rightmost column). For the former, many reported results can be found in the recent community white paper in Ref. [18], and we show here those lattice results that were designated as having "benchmark" status, where possible. Those lattice entries corresponding to single calculations are given with the associated reference, whereas those which result from an average of several lattice extractions are indicated with

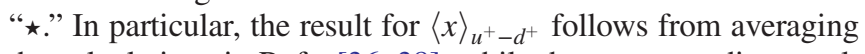
the calculations in Refs. [36-38], while the corresponding result for $\left\langle x^{2}\right\rangle_{u^{-}-d^{-}}$is an average over the result in Ref. [39] and two separate calculations reported in Ref. [40].

\begin{tabular}{lcc}
\hline \hline PDF moment & Lattice & CT14HERA2 NNLO \\
\hline$\langle x\rangle_{u^{+}-d^{+}}$ & $0.184(32) \star$ & $0.159(6)$ \\
$\left\langle x^{2}\right\rangle_{u^{-}-d^{-}}$ & $0.107(98) \star$ & $0.055(2)$ \\
$\left\langle x^{3}\right\rangle_{u^{+}-d^{+}}$ & N/A & $0.022(1)$ \\
$\langle x\rangle_{g}$ & $0.267(35)[36]$ & $0.415(8)$ \\
$\langle x\rangle_{u^{+}}$ & $0.453(75)[36]$ & $0.351(5)$ \\
$\langle x\rangle_{d^{+}}$ & $0.259(74)[36]$ & $0.193(6)$ \\
$\langle x\rangle_{s^{+}}$ & $0.092(41)[36]$ & $0.031(8)$ \\
$\left\langle x^{2}\right\rangle_{u^{-}}$ & $0.117(18)[41]$ & $0.085(1)$ \\
$\left\langle x^{2}\right\rangle_{d^{-}}$ & $0.052(9)[41]$ & $0.030(1)$ \\
$\left\langle x^{2}\right\rangle_{s^{-}}$ & N/A & $\ldots$ \\
$\langle 1\rangle_{\bar{d}-\bar{u}}$ & $\ldots$ & $-0.367(410)$ \\
$\kappa^{s}$ & $0.795(95)[42]$ & $0.459(132)$ \\
\hline \hline
\end{tabular}

at $90 \%$ C.L. in Table I. There is also an apparent tendency for the lattice-computed moments to overestimate the phenomenologically determined ones, a behavior that is likely especially driven by the effects of excited-state contamination. Despite this, however, we typically find reasonable alignment with lattice moments, partly owing to their larger uncertainties. As continued improvements tame the lattice effects described above, these uncertainties are expected to contract, leading to growing utility to global fits of unpolarized distributions. It is worth mentioning that, due to a comparatively smaller set of experimental data, the degree to which lattice calculations must improve to impact QCD analyses for spin-polarized quantities via Eqs. (7), (9), and (11b) is less pronounced.

\section{B. Analysis procedure}

\section{Correlations $\boldsymbol{C}_{f}$ and sensitivities $S_{f}$}

To explore the sensitivity of high-energy data to the PDF Mellin moments and qPDFs accessible in lattice QCD, in this work we extend the analysis of Ref. [22] that applied the recently developed PDFSense framework to weigh the impact of an extended amalgam of HEP experimental data (the "CTEQ-TEA," or "CT," data, plotted in Fig. 1) as a part of the preparation for the release of the CT18 PDF global analysis [43]. In this case, special emphasis was placed on the impact these data might have on the unpolarized collinear PDFs themselves and on observables derived directly therefrom, including the $14 \mathrm{TeV}$ inclusive Higgs production cross section, $\sigma_{H}$. We note that Fig. 1 is reproduced from Ref. [22] and provided here for ease of reference. Given the fact that a number of lattice QCD observables are calculable from phenomenological PDFs along the lines described in Secs. I, II A, and III below, we repeat our analysis to illustrate the constraints a typical experimental dataset can impose on our phenomenological knowledge of such lattice observables.

We refer the reader to Secs. II and III of Ref. [22] for a systematic presentation of the details of the PDFSense framework. Still, it is worthwhile to summarize the particulars of a sensitivity analysis dedicated to the PDF moments $\left\langle x^{n}\right\rangle_{q^{ \pm}}$. Whereas in Ref. [22] we primarily concentrated on the sensitivities of data to the local values of the collinear PDFs $q\left(x_{i}, \mu_{i}\right)$ at the typical $x_{i}$ and $\mu_{i}$ of the high-energy data points (see Appendix A of the same reference), here we are chiefly concerned with the sensitivity to Mellin moments for which the $x$ dependence has been integrated away, and in general at a scale $\mu_{F}=\mu^{\text {lat }}=2 \mathrm{GeV}$ at which moments are commonly computed in lattice QCD. Whether by a Hessian or Monte Carlo error procedure, a PDF global analysis typically produces a central PDF set and a finite ensemble replicas of the error PDFs, $q^{j \in\{2 N\}}\left(x, \mu_{F}\right)$. Given this ensemble, it is then possible to evaluate a respective error set for values of integrated PDF moments. In the case of the CT fitting approach, from the underlying Hessian error sets-of which there are $2 \mathrm{~N}$ for an $\mathrm{N}$-parameter global fit [leading to 1 (56) central (error) PDFs in the 28-dimensional CT14HERA2 NNLO fit]—we directly compute error replicas for the moments by integrating over the CT fitted distributions. Namely,

$$
\begin{aligned}
& q^{j \in\{2 N\}}\left(x, \mu^{\mathrm{lat}}\right) \longrightarrow\left\langle x^{n}\right\rangle_{q^{ \pm}, \mu^{\mathrm{at}}}^{j \in\{2 N\}} \\
& \quad=\int_{0}^{1} d x x^{n}\left(q\left(x, \mu^{\mathrm{lat}}\right) \pm \bar{q}\left(x, \mu^{\mathrm{lat}}\right)\right)_{j \in\{2 N\}} .
\end{aligned}
$$

In practice, we evaluate the integrals of Eq. (12) numerically from grids in which the bounds of integration are chosen to ensure stable convergence. For instance, we generally evaluate

$$
\int_{x_{a}}^{x_{b}} d x x^{n}\left(q\left(x, \mu^{\mathrm{lat}}\right) \pm \bar{q}\left(x, \mu^{\mathrm{lat}}\right)\right)_{j \in\{2 N\}},
$$

where typically we choose $x_{a} \sim 10^{-7}$ and $x_{b} \sim 0.999$. The stability of our results against variations about these choices has been explicitly verified. As can be seen in Fig. 1, the lowest reach of data in the CTEQ-TEA set is $x \sim 5 \times 10^{-5}$, such that the very low- $x$ contributions to the moments analyzed here are to be regarded as parametrization dependent. Generally, however, these contributions are heavily 


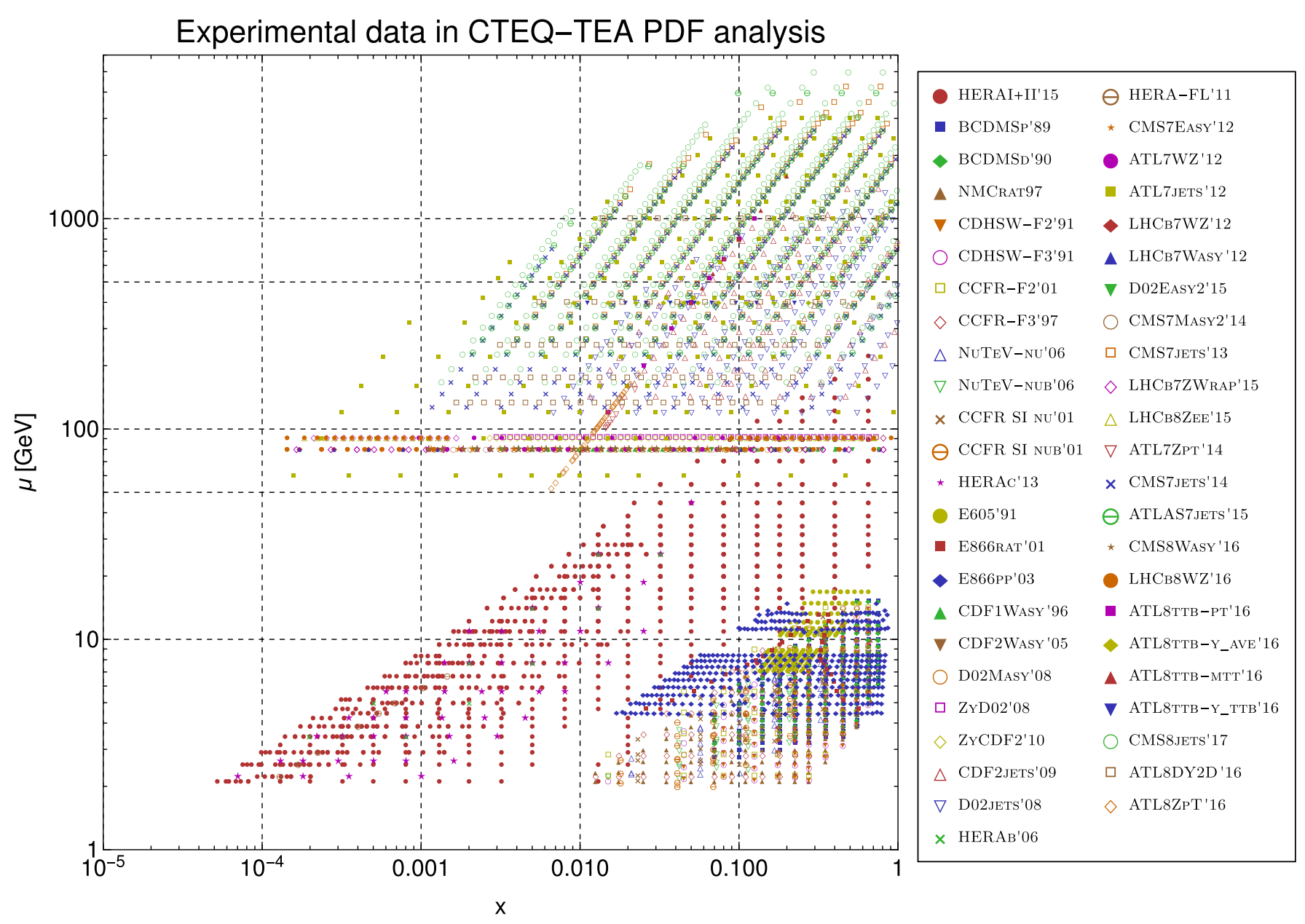

FIG. 1. A graphical representation of the space of $(x, \mu)$ points probed by the full dataset treated in Ref. [22] as well as the present analysis, designated "CTEQ-TEA." It corresponds to an expansion of the CT14HERA2 NNLO data fitted in the most recent CT14 framework, including measurements from Run II of HERA. We stress that the $x$ and $\mu$ which define the axes in this and subsequent plots represent the momentum fraction $x$ and scale $\mu$ matched from empirical data as described in Appendix A of Ref. [22]. These $(x, \mu)$ values are not to be confused with the $x$ of the qPDF in Eq. (2) or $\mu_{F}$ used to evaluate PDF moments. We direct the reader to the footnote near Eq. (2) for further clarification of this point.

suppressed in moments, $\left\langle x^{n}\right\rangle$, and the overall parametrization dependence is minimal.

With replica sets for lattice observables such as the PDF Mellin moments as in Eq. (12), we may deploy the statistical framework of Ref. [22], computing the Pearson correlation between the residual $r_{i}(\vec{a})$ of the $i$ th measurement of our CTEQ-TEA set [again, evaluated over the 1 (56) central (error) sets of CT14HERA2 NNLO] and the corresponding ensemble for $\left\langle x^{n}\right\rangle_{q^{ \pm}, \mu^{\text {lat }}}^{j \in\{2 N\}}$. The residual is defined by

$$
r_{i}(\vec{a})=\frac{1}{s_{i}}\left(T_{i}(\vec{a})-D_{i, s h}(\vec{a})\right)
$$

as the difference between the theoretical prediction $T_{i}(\vec{a})$, dependent on the PDF parameters $\vec{a}$, and central data value $D_{i}^{s h}(\vec{a})$ that is shifted to accommodate systematic error correlations. This difference is weighted by the total uncorrelated uncertainty, $s_{i}$ [44].

In practice, the correlation is computed using

$$
\begin{aligned}
C_{f}\left(x_{i}, \mu_{i}\right) & =\operatorname{Corr}\left[f, r_{i}\left(x_{i}, \mu_{i}\right)\right], \\
\operatorname{Corr}[X, Y] & =\frac{1}{4 \Delta X \Delta Y} \sum_{l=1}^{N}\left(X_{l}^{+}-X_{l}^{-}\right)\left(Y_{l}^{+}-Y_{l}^{-}\right),
\end{aligned}
$$

in which $f$ is a generic function of the PDFs [e.g., a PDF of given flavor at the matched $\left(x_{i}, \mu_{i}\right)$ of the $i$ th data point as in Ref. [22], or a PDF moment computed from the PDFs], and the $N l^{ \pm}$pairs of Eq. (15) may be identified with the $2 N$ Hessian error sets just described; the uncertainty quantities in the denominator of Eq. (15) are evaluated from the Hessian error sets as 


$$
\Delta X=\frac{1}{2} \sqrt{\sum_{l=1}^{N}\left(X_{l}^{+}-X_{l}^{-}\right)^{2}} .
$$

Equations (15) and (16) are motivated by the observation that, given the approximately Gaussian dependence of the probability distribution on the PDF parameters $\vec{a}$ in the close vicinity of the global minimum of $\chi^{2}$, small displacements of a PDF-dependent quantity $X$ from its value for the best-fit combination of PDF parameters can be computed using the $N$-dimensional vector of the gradient $\vec{\nabla} X$ with respect to $\vec{a}[44,45]$. Therefore, we can write, using the angle $\varphi$ between $\vec{\nabla} X$ and $\vec{\nabla} Y$,

$$
\begin{aligned}
\Delta X & =|\vec{\nabla} X|, \\
\operatorname{Corr}[X, Y] & =\cos \varphi=\frac{\vec{\nabla} X \cdot \vec{\nabla} Y}{\Delta X \Delta Y} .
\end{aligned}
$$

One of the principal results of Ref. [22] was the demonstration that the Pearson correlation of Eq. (15) cannot fully capture the phenomenological weight of individual measurements, given the fact that it does not explicitly depend upon the magnitudes of the PDF or experimental uncertainties. For this reason, we introduced a generalization of the correlation we call the sensitivity, $S_{f}$, of the $i$ th point in experiment $E$ to PDF flavor (or PDFderived quantity) $f$ :

$$
S_{f}=\frac{\Delta r_{i}}{\left\langle r_{0}\right\rangle_{E}} C_{f}
$$

$\Delta r_{i}$ is calculated using Eq. (16), and $\left\langle r_{0}\right\rangle_{E}$ represents the point-averaged residuals of each of the points of experiment $E$ computed with the central PDF set.

With the family of PDF error sets of CT14HERA2 NNLO and the statistical formalism and metric embodied by Eq. (18), the sensitivities of data points shown in Fig. 1 may be assessed and mapped in a plane of typical partonic momentum fraction $x_{i}$ and factorization scale $\mu_{i}$ at which a PDF, $q\left(x_{i}, \mu_{i}\right)$, might be evaluated. As developed in detail in Appendix A of Ref. [22], the matching of experimental data to specific values of $x_{i}$ and $\mu_{i}$ is based upon a leadingorder identification of $x_{i}$ and $\mu_{i}$ with scales dictated by external kinematics. For instance, in deeply inelastic scattering (DIS), we match the partonic fraction $x_{i}$ with Bjorken- $x_{B}:\left.x_{i} \approx x_{B}\right|_{i}$. Similarly, the QCD factorization scale associated with a specific DIS measurement is chosen to be the corresponding virtuality of the exchanged $\gamma$, $\left.\mu_{i} \approx Q\right|_{i}$. Analogous relations for data generated by the other processes appearing in Fig. 1, such as high-mass Drell-Yan scattering or jet production, appear in Appendix A of Ref. [22]. By mapping sensitivities in this fashion, we may isolate processes and individual datasets with a strong phenomenological pull on lattice observables, while also identifying the most constraining regions of $(x, \mu)$. The size of the absolute sensitivities $\left|S_{\left\langle x^{n}\right\rangle_{q^{ \pm}}}\right|$for highlighted points $\left(\left|S_{\left\langle x^{n}\right\rangle_{q^{ \pm}}}\right|>0.25\right)$ are identified by the "rainbow stripe" color palette: hot colors (red, orange) correspond to strong sensitivities, and cool colors (yellow, green) correspond to weak sensitivities. Unhighlighted points-i.e., those with relatively minimal expected impact, $\left(\left|S_{\left\langle x^{n}\right\rangle_{q^{ \pm}}}\right|<0.25\right)$, are represented with gray colors.

\section{The $L_{2}$ sensitivity}

The quantity $\left|S_{f}\right|$ defined in Eq. (18) is a convenient figure of merit that we will use to quantify the sensitivity of experimental data point residuals to the PDF dependence of select Mellin moments and qPDFs. It represents a normalized variation of the data residual $r_{i}$ when the PDF parameters are displaced along the direction of maximal variation of $f(x, Q)$, i.e., along $\vec{\nabla} f$, by an amount corresponding to the PDF error $\Delta f$.

To further elucidate the meaning of $S_{f}$, let us briefly discuss a related quantity, defined by

$$
\begin{aligned}
S_{f, L 2}(E) & \equiv \vec{\nabla} \chi_{E}^{2} \cdot \frac{\vec{\nabla} f}{|\vec{\nabla} f|} \\
& =\Delta \chi_{E}^{2}\left(\hat{a}_{f}\right)=\Delta \chi_{E}^{2} \cos \varphi\left(f, \chi_{E}^{2}\right) .
\end{aligned}
$$

In this equation,

$$
\chi_{E}^{2}(\vec{a})=\sum_{i=1}^{N_{p t}} r_{i}^{2}(\vec{a})+\sum_{\alpha=1}^{N_{\lambda}} \bar{\lambda}_{\alpha}^{2}(\vec{a})
$$

is the log-likelihood function for experiment $E$, equal to the quadratic sum ( $L_{2}$-norm sum) of $N_{p t}$ shifted data point residuals $r_{i}$ and $N_{\lambda}$ best-fit nuisance parameters $\bar{\lambda}_{\alpha}$ representing the sources of correlated systematic errors.

In accord with the discussion above, $S_{f, L 2}(E)$ is equal to $\Delta \chi_{E}^{2}\left(\hat{a}_{f}\right)$, the variation of $\chi_{E}^{2}$ when the PDF parameters are displaced by a unit vector $\hat{a}_{f} \equiv \vec{\nabla} f /|\vec{\nabla} f|$ along the gradient of $f$. As before, the displacement $\hat{a}_{f}$ from the best fit changes $f(x, Q)$ by its PDF error $\Delta f$. But the respective change $\Delta \chi_{E}^{2}\left(\hat{a}_{f}\right)$ is not equal to the PDF error $\Delta \chi_{E}^{2}$ on $\chi_{E}^{2}$. By applying Eqs. (17), one arrives at a different expression shown as the last equality on the second line of Eq. (19), i.e., a product of the PDF uncertainty $\Delta \chi_{E}^{2}$ estimated by Eq. (16), and the correlation cosine between $f(x, Q)$ and $\chi_{E}^{2}$ given by Eq. (15).

Thus, $S_{f, L_{2}}(E)$ measures the $\chi^{2}$ change for experiment $E$ when the PDF parameters are displaced from their best-fit value so that $f(x, Q)$ at the chosen $x$ and $Q$ is increased by its Hessian PDF error, which we take to correspond to the $68 \%$ probability level (p.l.) in this presentation. By computing $S_{f, L 2}(E)$ for every fitted experiment $E$, we can easily compare the pulls of the individual experiments on $f(x, Q)$, solving essentially the same task as a Lagrange multiplier 
scan on $f(x, Q)$ [46], but extracting most of the same information much faster, within the limits of the Hessian approximation. This approach can explore the consistency of individual experiments along much the same lines as the Hessian dataset diagonalization technique [47], with the advantage that it performs this analysis along multiple directions in the Hessian PDF parameter space at once. We may call $S_{f, L 2}(E)$ as the $L_{2}$ (norm) sensitivity, to distinguish it from the $L_{1}$ (norm) sensitivity $S_{f}$ that is constructed from the residuals themselves, rather than from their squares.

Figure 2 illustrates the application of the $L_{2}$ sensitivity to the CTEQ-TEA experimental datasets that were selected for the upcoming CT18 global fit. We compute the $L_{2}$ sensitivity at the lattice scale $\mu=2 \mathrm{GeV}$ for the isovector combination $u^{+}(x, \mu)-d^{+}(x, \mu)$. Due to the dependence of $S_{f, L_{2}}$ on $\cos \varphi\left(f, \chi_{E}^{2}\right)$, it concisely represents the competing pulls of the CTEQ-TEA datasets. The respective $\Delta \chi_{E}^{2}\left(\hat{a}_{f}\right)$ can be either correlated $\left(S_{f, L_{2}}>0\right)$ or anticorrelated $\left(S_{f, L_{2}}<0\right)$ with $u^{+}(x)-d^{+}(x)$. Large experimental errors or weak correlations correspond to $S_{f, L_{2}}(E) \approx 0$ and imply vanishing pulls for a particular experiment on the PDF at a given $x$.

In Fig. 2, we included only the curves for 12 experiments with the highest $L_{2}$ sensitivities, selected out of 40 eligible experiments by the requirement that the shown experiments must have $\left|S_{f, L_{2}}(E)\right|>4$ in some interval of $x$. Recall that the textbook parameter-fitting criterion associates $\Delta \chi_{E}^{2}=1$ (or 4 ) with the uncertainty at the $68 \%$ (or $95 \%$ ) probability level. The $L_{2}$ sensitivity plots such as the one shown in

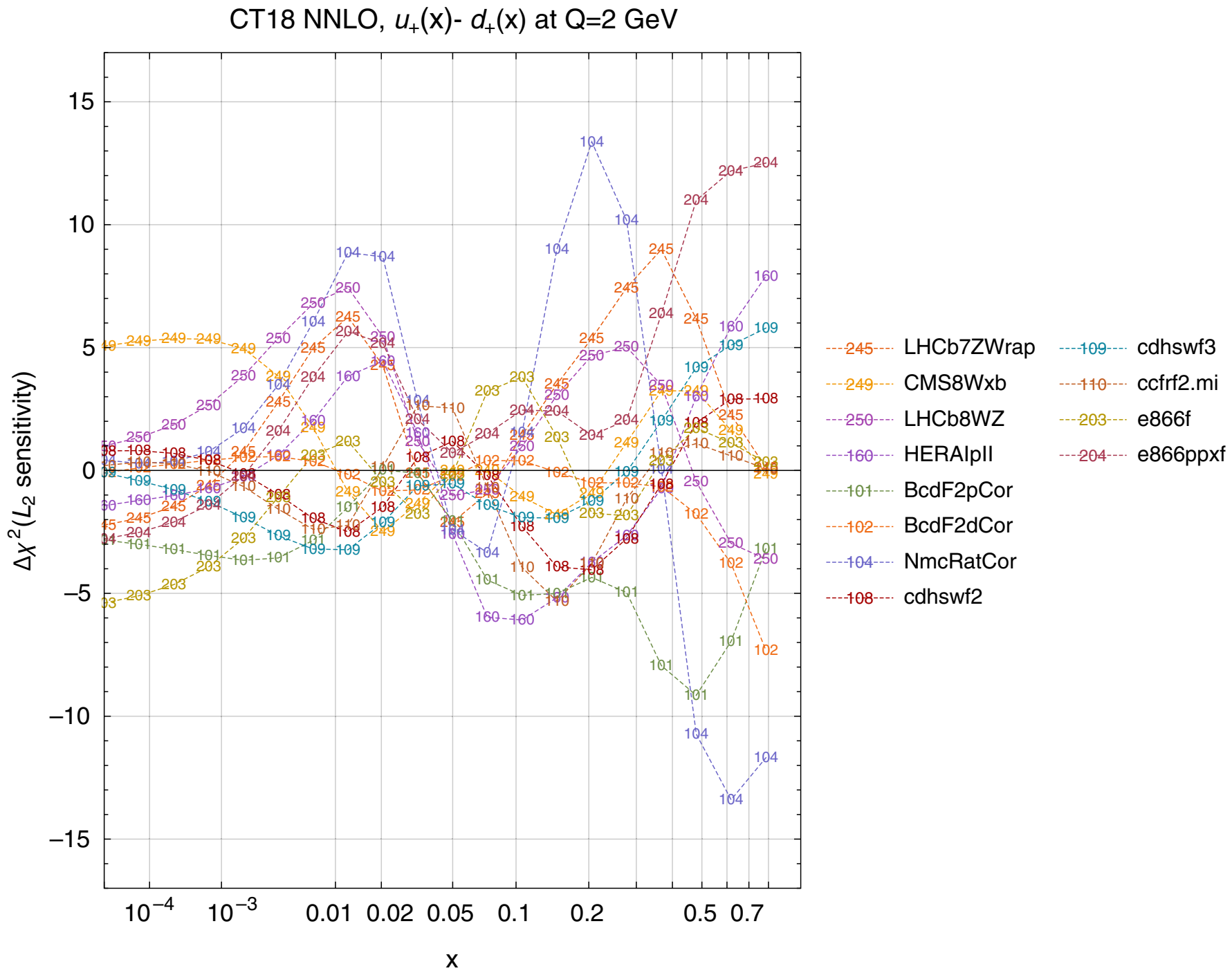

FIG. 2. The $L_{2}$ sensitivities of the CTEQ-TEA with the strongest pulls on the isovector PDF combination, $u^{+}(x)-d^{+}(x)$, at the scale $\mu=2 \mathrm{GeV}$ as computed using Eq. (19). Here, we evaluate the $L_{2}$ sensitivity using a subset of the CTEQ-TEA data prepared for the CT18 NNLO PDF fit [43], based on the implementation of LHC Run-1 data explored in Ref. [22]. The curves for the fitted experiments reveal a number of competing pulls on the isovector $q^{+}$distribution: for instance, at $x>0.1$, between the E866 $p p$ (204), combined HERA (160), and CDHSW (109) datasets, on the one hand, and the NMC $F_{2}^{d} / F_{2}^{p}$ ratio measurements (104) and BCDMS $F_{2}^{d}$ data (102), on the other. 
Fig. 2 reveal far larger latent tensions between the experiments in some $x$ regions, evident as deviations in opposite directions with magnitudes $\left|S_{f, L_{2}}(E)\right|>5$. That is, the $\chi^{2}$ value changes by more than five units when the PDFs are varied by the $68 \%$ p.l. error along the respective direction. In the region $x>0.1$ that contributes the most to the Mellin moments, Fig. 2 suggests that the sharpest positive pulls on the $u^{+}(x)-d^{+}(x)$ distribution come from the E866 $p p$ (204), combined HERA (160), and CDHSW (109) data, whose $\chi_{E}^{2}$ are positively correlated with the isovector PDF, and the opposite pulls by the NMC ratio measurements (104) and BCDMS $F_{2}^{d}$ data (102), for which the $L_{2}$ sensitivity is negative. Similar tensions exist at smaller values of $x$ as well.

The upshot of the figure is that a variety of DIS and Drell-Yan experiments on nucleon and nuclear fixed targets continue to play the prominent role in constraining the isovector combination, surpassing in this regard the available LHC measurements that still are to grow in their importance. Similar observations apply to the Mellin moments and qPDFs that we will consider. The sensitivity studies, as well as related methods such as the Lagrange multiplier scan, reveal tensions among the miscellaneous experiments in the CTEQ-TEA dataset that constrain the PDFs at $x>0.1$. The $x$-dependent $L_{2}$ sensitivity shown in Fig. 2 should be consulted in parallel with the $S_{f}$ sensitivity calculations shown for the moments and quasi-PDFs in the sections below.

\section{Structure of the presentation}

In the next subsection, we will compute and investigate the $S_{f}$ sensitivity of the CTEQ-TEA dataset to the lowest moments of the unpolarized light-quark and gluon distributions, $\left|S_{\left\langle x^{n}\right\rangle_{q^{ \pm}, g}}\right|$, in the $(x, \mu)$ plane with the PDFSense package [22]. We use the CT14HERA2 NNLO PDF set [5] in the theoretical predictions and residuals of experimental data. Our dataset measurements in the CT14HERA2 NNLO fit and the latest LHC jet, $t \bar{t}, W / Z$ production datasets.

The sensitivities from the existing experiments discussed in this section can be further confronted by the projected sensitivities to the same PDF-dependent quantities in Sec. IV for a set of pseudodata for inclusive neutral-current (NC) and charge-current (CC)-mediated $e^{ \pm} p$ DIS at a future EIC-like collider. The scientific program at an electron-ion collider (EIC) or a similar machine is anticipated to significantly complement lattice QCD in learning about the three-dimensional structure of hadrons. The procedure of the preliminary impact study in Sec. IV is broadly similar to that for the analysis of the real CTEQ-TEA data, but is based on the DIS pseudodata obtained by generating Gaussian fluctuations about the CT14HERA2 NNLO theoretical prediction for the reduced cross sections according to an assumed precision.
The flavor combinations primarily discussed in this paper are $u^{ \pm}-d^{ \pm}, u^{ \pm}, d^{ \pm}, s^{+}$, and $g$ in which $q^{+}$here refers to the $\mathrm{C}$-even combination of (anti)quark distributions, $q+\bar{q}$; correspondingly, we also consider C-odd quantities, $q^{-}=q-\bar{q}$, as defined in Eqs. (3) and (4). The PDF moment(s) for the light flavor combinations $u^{ \pm}-d^{ \pm}, u^{ \pm}, d^{ \pm}$, and $s^{+}$are computed on the lattice by the $\mathcal{O}_{\left\{\mu_{1}, \ldots, \mu_{n}\right\}}^{F}$ operators noted above in Eq. (8), whereas for the gluon distribution $g$, the operator noted immediately after Eq. (11) is required. The present status of the lattice QCD calculations of these parton moments is widely varied, with some moments (especially for the isovector combination $u-d$ ) evaluated by multiple groups with various systematic treatments; on the other hand, lattice information on the second moments of the individual lightquark flavors $\left\langle x^{2}\right\rangle_{q^{-}}$, for instance, is comparatively sparse. At the same time, the corpus of lattice computations is growing, and the availability of calculations for the moments considered here (and beyond) will increase.

We note that many numerical results for PDF moments computed both on the lattice and from different QCD global analyses are detailed in Appendixes B and C of the recent white paper in Ref. [18].

\section{Numerical results}

We now present the calculated sensitivity maps for the CTEQ-TEA data to each of the lowest moments of the light-quark $(u-d, u, d, s)$ distributions and the gluon. We also examine the aggregated impact of the experiments in the CTEQ-TEA set on each of these quantities and consider the implications for unraveling the nucleon's flavor structure and benchmarking lattice QCD output of the same objects.

\section{Moments of nucleon quark distributions}

a. Moments of isovector flavor distributions.-Historically, computation of isovector PDF combinations in SU(2) isospin space has represented an especially fertile proving ground for lattice gauge methods-particularly given that gluon and singlet quark densities mix evenly with $u^{+}$and $d^{+}$distributions under DGLAP evolution, such that $u^{+}-$ $d^{+}$has a nonsinglet scale dependence. A consequence specific to lattice QCD is the fact that contributions from disconnected insertions vanish in the difference (assuming parton-level charge symmetry), and a much less computationally costly calculation based purely on connected insertions is generally adequate. For this main reason, the isovector combinations have been a focus of lattice calculations for both the PDF Mellin moments-and, more recently, the quasidistributions in both the nucleon [48-53] and the pion [54-57].

We plot the sensitivity map of the CTEQ-TEA data to two of the lower moments of the nucleon isovector distribution in Fig. 3-namely, the sensitivities to the total 
$\left|S_{f}\right|$ for $\left\langle x^{1}\right\rangle_{u^{+}-d^{+}}$, CT14HERA2

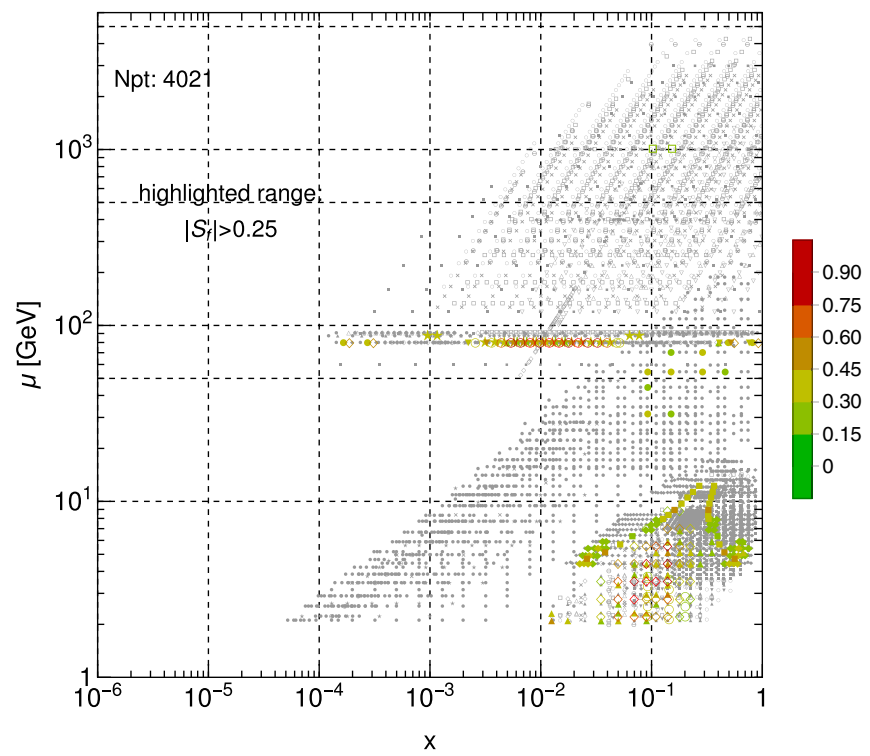

$\left|S_{f}\right|$ for $\left\langle x^{2}\right\rangle_{u^{-}-\alpha^{+}}$, CT14HERA2

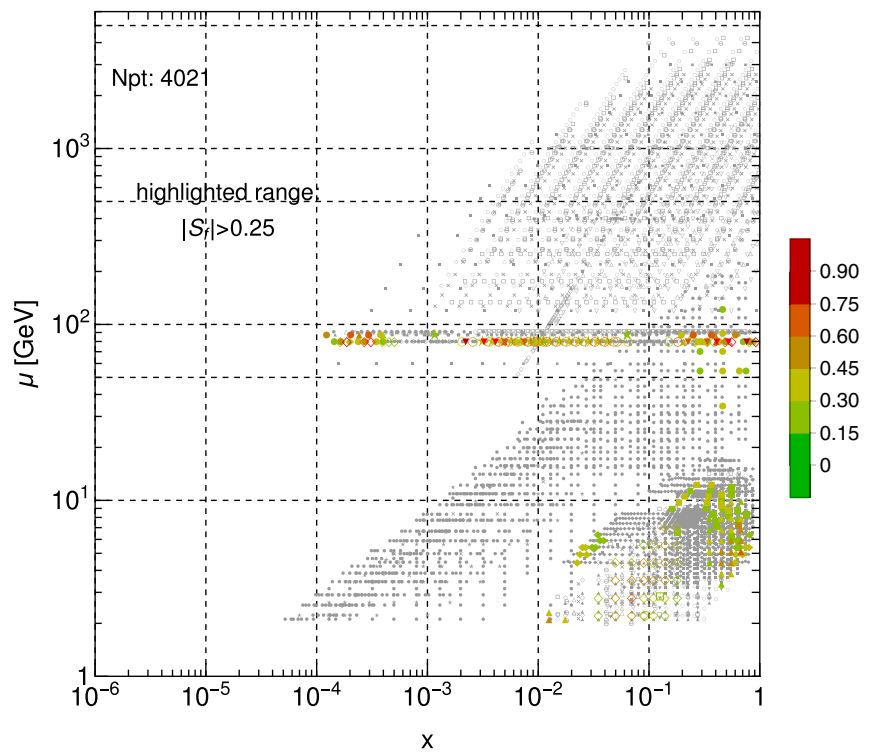

FIG. 3. Sensitivity of the CTEQ-TEA datasets to $\langle x\rangle_{u^{+}-d^{+}}$and $\left\langle x^{2}\right\rangle_{u^{-}-d^{-}}$. The factorization scales of Mellin moments and PDFs are $\mu_{F}=\mu^{\text {lat }}=2 \mathrm{GeV}$. As described in the text below, we colorize only those data points with significant individual sensitivity $\left|S_{f}\right|>0.25$, so as to highlight the pulls of the most constraining measurements. Points falling below this highlighting threshold (i.e., with $\left.\left|S_{f}\right|<0.25\right)$ are indicated in grayscale.

isovector momentum $\langle x\rangle_{u^{+}-d^{+}}$(left panel) and the secondorder asymmetry moment $\left\langle x^{2}\right\rangle_{u^{-}-d^{-}}$(right panel). These plots have the same basic configuration as developed in Ref. [22], with emphasis placed on specific measurements of individual experimental datasets with particularly strong pulls in the global analysis. The predicted pull of these measurements as quantified by the sensitivity $\left|S_{f}\right|$ is represented by the color scheme shown in the offset to the right for each panel in Fig. 3. As in Ref. [22], we draw attention to the most impactful data and physical processes by imposing a highlighting cut, $\left|S_{f}\right|>0.25$, and selecting a coloration scheme which suitably reveals the dependence of data sensitivities on the kinematical matching parameters. On the basis of our sensitivity analysis, we are in a position to make a number of observations regarding the empirical information that drives the current knowledge of the lower $\left\langle x^{n}\right\rangle_{u-d}$ Mellin moments.

The highlighted points emphasized in the panels of Fig. 3 often rather closely correspond to the CTEQ-TEA experiments which enjoy the highest per-datum sensitivities,

$$
\left\langle\left|S_{f}\right|\right\rangle=\frac{1}{N_{p t}} \sum_{i \in N_{p t}}\left|S_{f}^{i}\right| .
$$

In decreasing order, these are CMS7Masy2'14 (0.557), E866rat'01 (0.365), CMS7Easy'12 (0.333), CCFR-F3'97 (0.307), and NMCrat'97 (0.212), where the quantity in parentheses is the computed average of each experiment per measured point.
For the purpose of enumerating this information, we include only those experimental measurements with pointaveraged sensitivities exceeding the lower bound $\left\langle\left|S_{f}\right|\right\rangle>$ 0.2 . On the other hand, by the total sensitivity metric $\sum_{i \in N_{p t}}\left|S_{f}^{i}\right|$, we identify a somewhat different collection of experiments with a leading impact on the first isovector moment; viz. HERAI + II'15 (37.8); CCFR-F3'97 (26.4); NMCrat'97 (26.1); E866pp'03 (20.7); BCDMSp'89 (19.3). We point out that the point-averaged and total sensitivities for all CTEQ-TEA experiments are summarized in Appendix using ranking tables, as well as in Figs. 13 and 14, which are further described below. Additional numerical details can be found on the companion website, Ref. [58]. In this context, there is a pronounced influence of the combined HERAI + II experiment due to the extremely large number of measurements $\left(N_{p t}=1120\right)$ taken-and despite the fact that only a minimal number of these exhibit per-point sensitivities that exceed the highlighting cut $\left|S_{f}\right|>0.25$ imposed on the impact maps in this analysis.

Continuing, the right panel of Fig. 3 also shows the corresponding distribution of CTEQ-TEA sensitivities in $(x, \mu)$ space for the second isovector moment $\left\langle x^{2}\right\rangle_{u^{-}-d^{-}}$, for which the constraints arising from individual experiments fitted by CT are somewhat different. In this instance, we find the distribution of point-averaged sensitivities within the CTEQ-TEA dataset to be driven primarily by electroweak boson production measurements: CMS7Masy2'14 (0.492), D02Easy2'15 (0.416), CMS7Easy'12 (0.282), LHCb7Wasy'12 (0.250), CCFR-F3'97 (0.224), and 
D02Masy'08 (0.211). In contrast to the total isovector momentum considered above, we therefore again find a leading role for the $7 \mathrm{TeV}$ CMS lepton asymmetry measurements of $A_{\mu}(\eta)$ [CMS7Masy2'14] and $A_{e}(\eta)$ [CMS7Easy'12], although the size of the sets $\left(N_{p t}=11\right)$ is such that their aggregated pull on $\left\langle x^{2}\right\rangle_{u^{-}-d^{-}}$is dominated by larger fixed-target datasets identified by an analysis of the summed sensitivities, as we point out below. In addition to the CMS measurements, a number of other electroweak boson sets evidently have a stronger pull on the $\left\langle x^{2}\right\rangle$ isovector moment, including the corresponding D0 measurement of $A_{e}(\eta)$ (D02Easy2'15), which probes higher $x$, as well as LHCb.

The evaluation according to the aggregated sensitivities reveals a different hierarchy. In this case, fixed-target measurements of DIS cross sections and structure functions-as well as a couple Drell-Yan sets-are dominant, namely, HERAI + II'15 (36.5), BCDMSp'89 (33.1), E866pp'03 (22.2), CCFR-F3'97 (19.3), and NMCrat'97 (18.4), with a rapid falloff in the aggregated sensitivity below $\sum\left|S_{f}\right|$ beyond these experiments. It should be noted, however, that were the boson production datasets with especially strong per-datum sensitivities indicated above combined into a single collection, the resulting aggregated impact of this collection would approach $\sum\left|S_{f}\right| \sim 34$, placing this combination of 150 data points just beyond the BCDMS $F_{2}^{p}$ data $\left(N_{p t}=337\right)$ and only behind the HERAI + II' 15 set $\left(N_{p t}=1120\right)$ in total sensitivity.

b. Moments of $q^{+}$distributions.-As we pointed out in the discussion of the $u-d$ moments at the start of Sec. II D 1 above, the fact that the disconnected insertions contribute equally to $u$ - and $d$-type distributions implies their vanishing for isovector $\left(\tau_{3}\right)$ charges. Unlike these combinations, the moments of flavor-separated distributions such as $u^{+}\left(x, \mu^{\text {lat }}\right)$ and $d^{+}\left(x, \mu^{\text {lat }}\right)$ receive contributions from both connected and disconnected insertions. The disconnected insertions arise from Wick contractions of quark fields not explicitly present in interpolation operators used to construct the 2-point function associated with the nucleon propagator; disconnected insertions are therefore essentially equally present in both $u$-type and $d$-type flavor-separated moments. Unfortunately, evaluating disconnected insertions on the lattice is computationally expensive and, historically, has proved challenging.

In the case of the higher moments, they are generally quite small-e.g., Ref. [41] found $\left\langle x^{2}\right\rangle_{u^{-}, d^{-}}$to be consistent with zero, and, along these lines, the disconnected contributions in these instances will themselves be fairly small. In fact, even for the larger first moments $\langle x\rangle_{q}^{+}$, the differences between calculations with and without disconnected insertions are within uncertainties, suggesting that these contributions may not be so large for the $u$ - and $d$-type distribution moments. Nucleon strangeness, on the other hand, necessarily originates exclusively with disconnected insertions, since the proton possesses no valence strange content, and, consequently, no strange quark fields are explicitly present in the nucleon interpolation operators from which two-point correlation functions are evaluated. Precise lattice data involving each of these flavors as well as for multiple Mellin moment orders would be instrumental in disentangling the interplay of connected vs disconnected insertions and helping to resolve the underlying dynamics. This observation also motivates a comprehensive assessment of the same moments as computed from phenomenological PDFs as well as a reckoning of the various pulls from experimental data that act upon them.

$u^{+}$-quark moments. For $\langle x\rangle_{u+}$, we consider the CTEQTEA sensitivity contained in the map of the LHS panel of Fig. 4; as is the case fairly generically for the leading moments of the light-quark distributions, the most concentrated locus of high-sensitivity data is found in the fixed-target sector in the lower right quadrant of the $(x, \mu)$ plot-particularly for $x \gtrsim 0.01$ and $\mu \lesssim 10 \mathrm{GeV}$. Upon inspection, these points arise from measurements at BCDMS (on the proton-BCDMSp'89-as well as the deuteron, BCDMSd'90) and the E866 data. Empirical information with especially larger per-datum sensitivities can again be identified by listing the leading experiments in descending order of their point-averaged sensitivities. These are CCFR-F3'97 (0.337), E866rat'01 (0.277), D02Masy'08 (0.250), CMS7Masy2'14 (0.248), and NuTeV-nu'06 (0.221). While for the total sensitivities we find HERAI + II'15 (40.8), BCDMSp'89 (39.5), CCFRF3'97 (29.0), BCDMSd'90 (24.8), CDHSW-F2'91 (16.5), CDHSW-F3'91 (15.1), and E866pp'03 (10.3).

$d^{+}$-quark moments. As an illustration of the flavor dependence of the PDF moments, we compare the right panel of Fig. 4 with the corresponding sensitivities for $\langle x\rangle_{d+}$, shown in the right panel. Here we find again a strong role for charged-current processes from lepton charge asymmetry data and $\nu A$ DIS, if the leading per-datum sensitivities are considered: CMS7Masy2'14 (0.419), NuTeV-nu'06 (0.238), CMS7Easy'12 (0.228), CCFRF3'97 (0.227), and CDHSW-F2'91 (0.225). On the basis of the total sensitivities of these experiments, however, we again find a hierarchy dominated by the combined HERA dataset, for which the $\mathrm{CC} e^{ \pm} p$ channels show somewhat enhanced sensitivity to moments of $d(x)$ relative to $u(x)$ according to both the $\left\langle\left|S_{f}\right|\right\rangle$ and $\sum\left|S_{f}\right|$ metrics illustrated in Fig. 13; this is particularly true of the CC $e^{+} p$ HERAI + II information, for which the LO reduced cross section $\sigma_{r}\left(x, Q^{2}\right)$ is closely driven by the behavior of $d$-type quark distributions, especially at larger $x$. Beyond the HERA measurements, the descending list of the experiments with high total sensitivities has a trailing collection of fixedtarget measurements, namely, HERAI + II'15 (54.2), BCDMSd'90 (26.5), NMCrat'97 (22.6), CCFR-F3'97 (19.5), CDHSW-F2'91 (19.1), BCDMSp'89 (18.5), and 
$\left|S_{f}\right|$ for $\left\langle x^{1}\right\rangle_{u^{+}}$, CT14HERA2

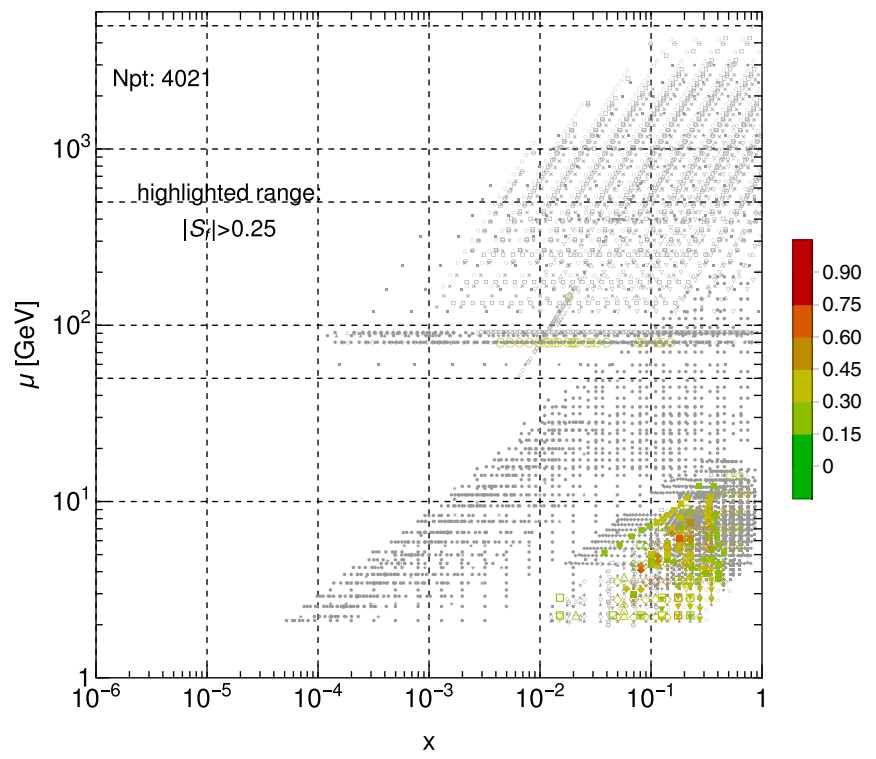

$\left|S_{f}\right|$ for $\left\langle x^{1}\right\rangle_{d^{+}}$, CT14HERA2

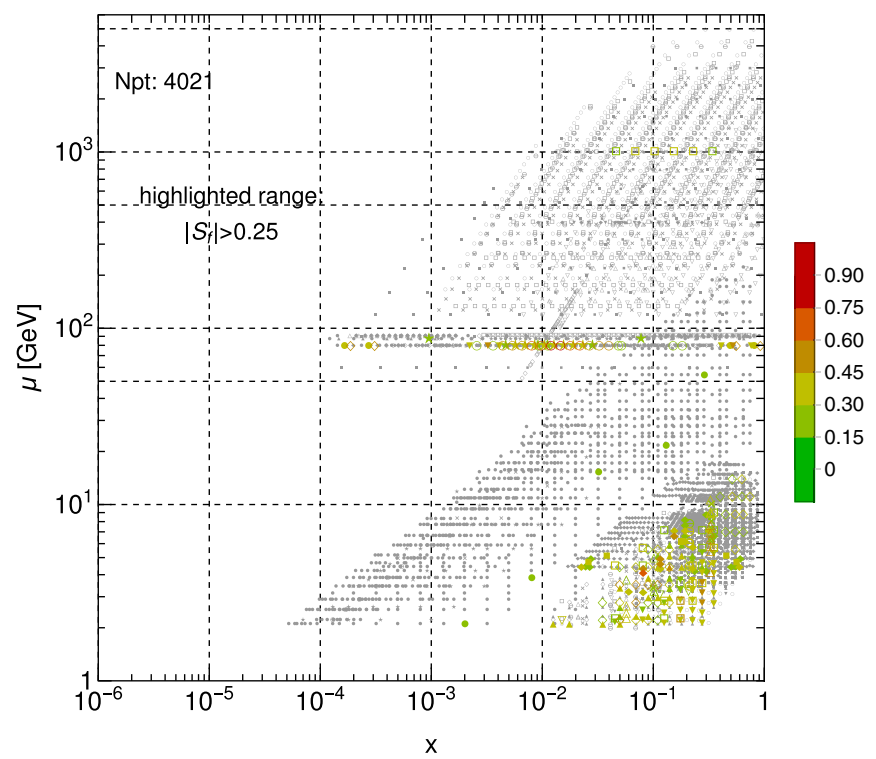

FIG. 4. Sensitivity of the CTEQ-TEA datasets to $\langle x\rangle_{u^{+}}$(left panel) and $\langle x\rangle_{d^{+}}$(right panel). As in Fig. 3, sensitivities are with respect to moments evaluated at $\mu_{F}=\mu^{\text {lat }}=2 \mathrm{GeV}$.

E866pp'03 (14.8). In this instance, the second most influential measurement is the deuteron target structure function extractions from BCDMS (BCDMSd'90)—a fact consistent with the traditional importance ascribed to deuteron measurements for performing nucleon flavor separations.

$s^{+}$-quark moments. The sensitivities to the moments of the $s^{+}$distribution are presented in Fig. 5. For $\langle x\rangle_{s^{+}}$, the measurements with leading point-averaged sensitivities are found to be NuTeV-nu'06 (0.429), CCFR SI nub'01 (0.344), CCFR SI nu'01 (0.313), NuTeV-nub'06 (0.302), and D02Masy'08 (0.274); while those with the highest predicted total impact based on aggregated sensitivity are HERAI + II'15 (31.4), NuTeV-nu'06 (16.3), CCFR SI nub'01 (13.1), CCFR SI nu'01 (12.5), and NuTeVnub'06 (10.0). Across both the aggregated and the average

\section{$\left|S_{f}\right|$ for $\left\langle x^{1}\right\rangle_{s^{+}}$, CT14HERA2}

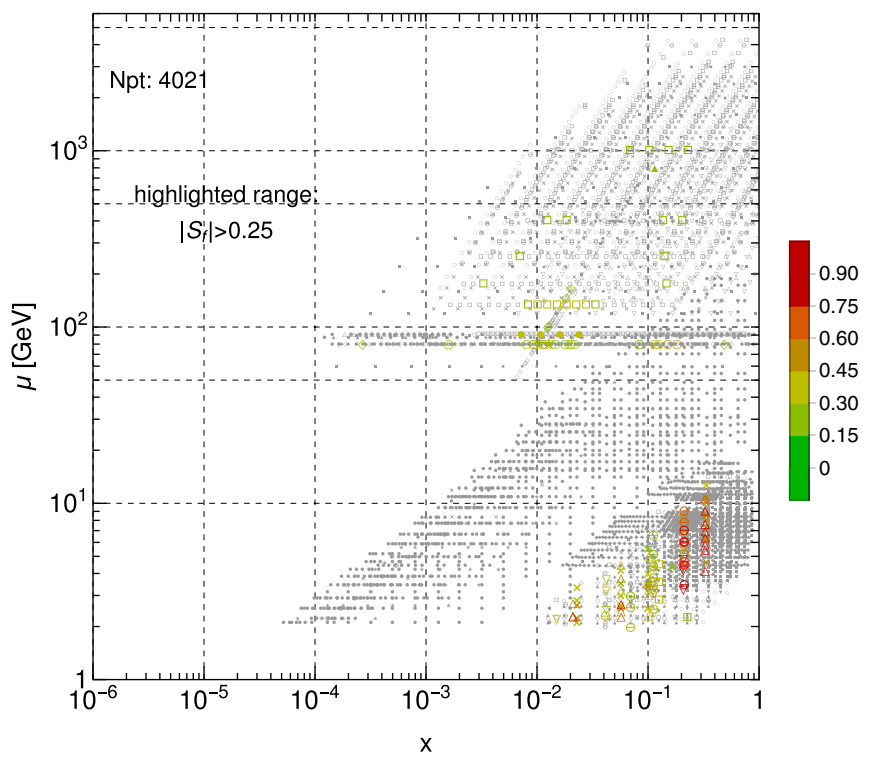

\section{$\left|S_{f}\right|$ for $\left\langle x^{3}\right\rangle_{s^{+}}$, CT14HERA2}

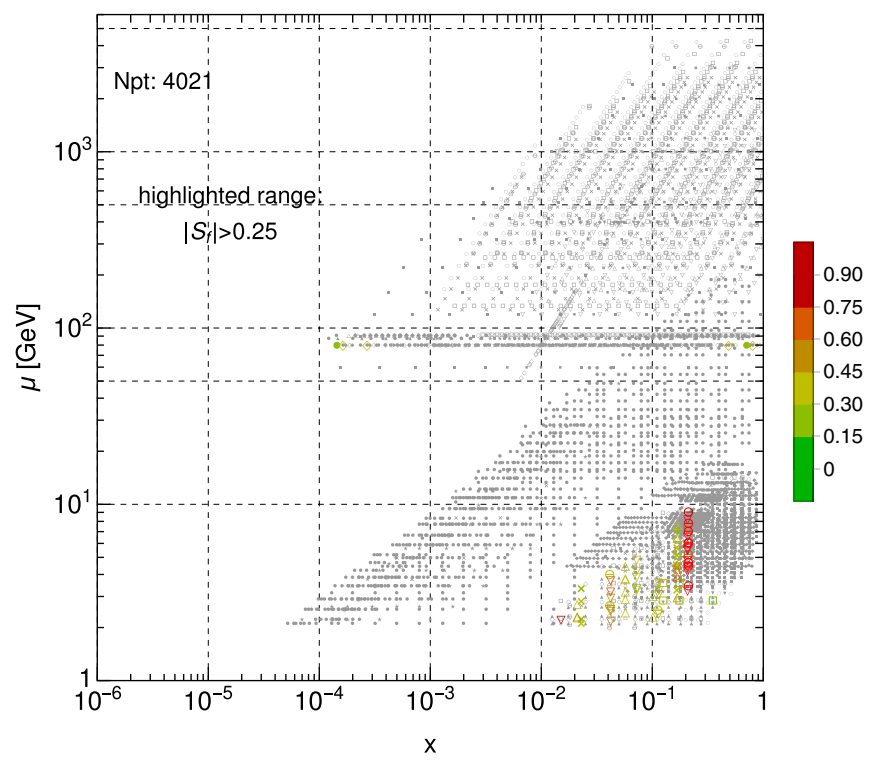

FIG. 5. CTEQ-TEA sensitivity to the first- and third-order Mellin moments of the $s^{+}$distribution. As elsewhere, the factorization scales of Mellin moments and PDFs are $\mu_{F}=2 \mathrm{GeV}$. 
per-point sensitivities, the decisive role of neutrino scattering data is evident, despite the still leading role of the combined HERA measurements - especially noting the fact that the summed sensitivity of the four leading $\nu$ experiments mentioned above is $\sum\left|S_{f}^{\nu}\right|=51.9$, exceeding the HERA accumulated impact by $\sim 65 \%$.

In the CT14HERA2 NNLO PDF set, strangeness was parametrized symmetrically (i.e., under the assumption $s(x)=\bar{s}(x)$; as a result, the moments of the $s^{-}$-type distributions, including $\left\langle x^{2}\right\rangle_{s^{-}}$, are identically zero. For that reason, we instead consider here the next highest moment of the strangeness distribution, i.e., the third moment $\left\langle x^{3}\right\rangle_{s^{+}}$; for which we find the point-averaged sensitivities of the leading experiments (again, cutting at $\left\langle\left|S_{f}\right|\right\rangle>0.2$ ) to be NuTeV-nub'06 (0.568), CCFR SI nub'01 (0.387), and NuTeV-nu'06 (0.269), clearly suggesting the very important role of the $\mathrm{NuTeV} \bar{\nu}$ dimuon production measurements (NuTeV-nub'06), which show especially enhanced sensitivity to the higher $\left\langle x^{3}\right\rangle_{s^{+}}$moment than was seen for the total strange momentum $\langle x\rangle_{s^{+}}$. For the total sensitivities, the constraints imposed by the CTEQ-TEA dataset come primarily from several experiments HERAI + II'15 (20.3), NuTeV-nub'06 (18.7), CCFR SI nub'01 (14.7), and NuTeV-nu'06 (10.2). Thus, for both Mellin moments of the $s^{+}$distribution, the fixed-target $\nu$ DIS experiments enjoy a clear advantage in their sensitivity compared to the rest of the CT14HERA2 experimental datasets.

c. Moments of $q^{-}$distributions.-At present, lattice determinations for the next highest $\left\langle x^{2}\right\rangle_{q}$ moments of the lightquark distributions have not matured to the level of extant calculations of the first moments $\langle x\rangle_{q}$, particularly in the

\section{$\left|S_{f}\right|$ for $\left\langle x^{2}\right\rangle_{u^{-}}$, CT14HERA2}

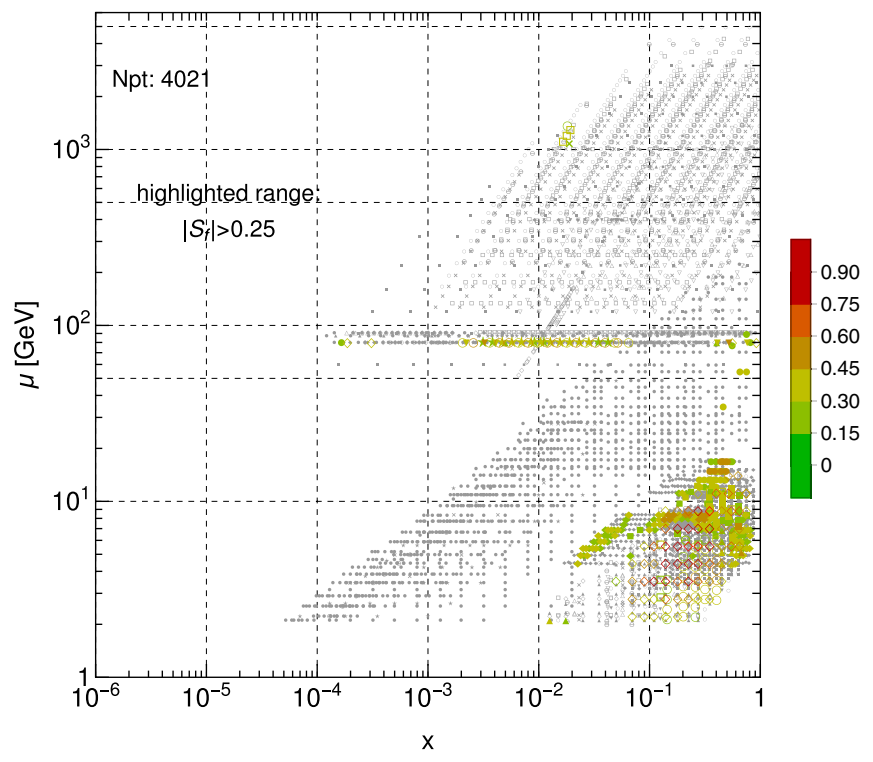

sense that these have been computed thus far only in Ref. [41] in the quenched approximation (i.e., excluding dynamical quark loops). Nonetheless, such determinations are likely forthcoming and can yield vital information regarding asymmetric $x$ dependence in the light-quark distributions.

We plot the sensitivity maps to the $\left\langle x^{2}\right\rangle_{q^{-}}$moments of the $u$ - and $d$-quark distributions in the left and right panels of Fig. 6, respectively. As elsewhere, these panels examine the sensitivity of the CTEQ-TEA set to moments evaluated at the typical lattice scale $\mu=\mu^{\text {lat }}=2 \mathrm{GeV}$.

$u^{-}$-quark moments. For the second moment of the $u^{-}$ distribution, the leading point-averaged sensitivities are due to fixed-target DIS experiments and the $7 \mathrm{TeV}$ CMS lepton charge asymmetries, led by CCFR-F3'97 (0.503); beyond this, experiments with $\left\langle\left|S_{f}\right|\right\rangle>0.2$ are CMS7Masy2'14 (0.413), CDHSW-F3'91 (0.248), and CMS7Easy'12 (0.244). In this context, the fact that information on the parity-odd structure function $F_{3}^{p}$-especially as provided by CCFR-F3' 97 - shows such sizable influence over $\left\langle x^{2}\right\rangle_{u^{-}}$ is consistent with the leading-order $\sim q-\bar{q}$ behavior of $F_{3}^{p}$ in the quark-parton model. As such, thorough knowledge of the $x$ dependence of $x F_{3}$ facilitates an unraveling of the $C$-odd distributions of the $q^{-}$type and constrains their higher moments. As was the case, however, for the $\langle x\rangle_{q^{+}}$ moments, consideration of the aggregated sensitivities reveals a larger spread of experiments with the strongest pulls belonging again to the combined HERA dataset HERAI + II'15 (43.9), the $\nu$ DIS measurements of $x F_{3}$ from CCFR [CCFR-F3'97 (43.2)] identified by the pointaveraged ranking above, BCDMSp'89 (39.2), and E866pp'03 (32.7). Having somewhat diminished but still significant pulls are several of the other fixed-target

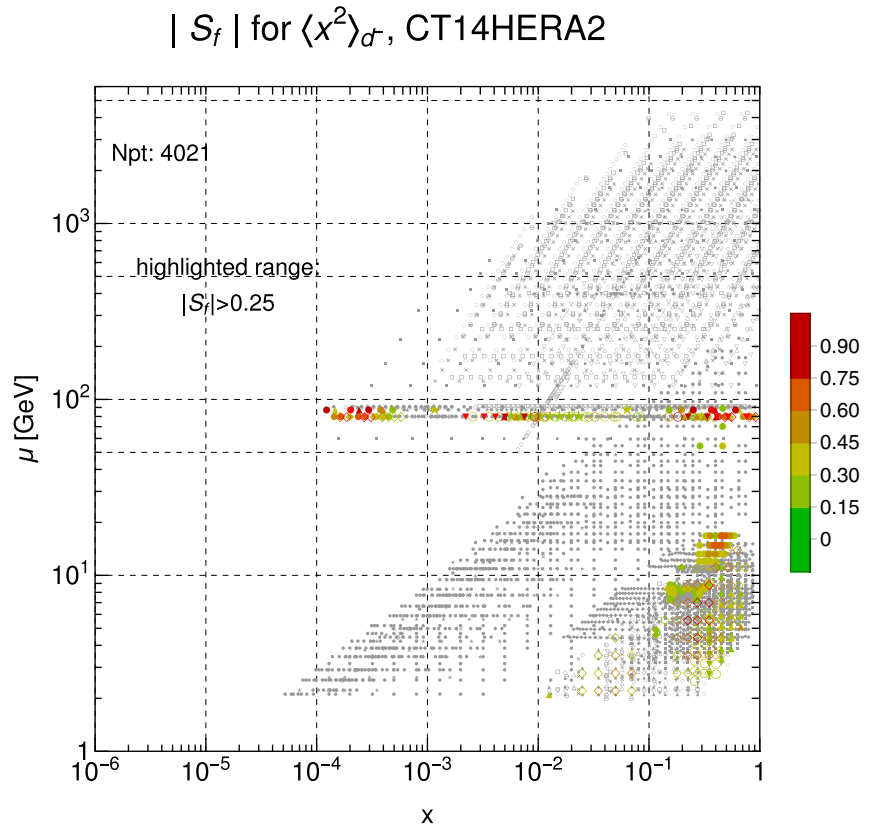

FIG. 6. Sensitivity of the CTEQ-TEA data to the $v_{u, d}^{3}$ moments $\left\langle x^{2}\right\rangle_{u^{-}}$(left) and $\left\langle x^{2}\right\rangle_{d^{-}}$(right), computed for $\mu_{F}=2 \mathrm{GeV}$. 
experiments involving both neutrino and $\mu$ DIS as well as the Drell-Yan process; namely, these are CDHSW-F3'91 (23.8), E605'91 (18.4), BCDMSd'90 (13.6), and NMCrat'97 (13.4).

$d^{-}$-quark moments. As observed above for the lower $\langle x\rangle_{q^{+}}$moments imaged in Fig. 4, there are evident differences between the sensitivity maps for $d$-vs $u$-quark moments, and this holds again for the explicit comparison of $\left\langle x^{2}\right\rangle_{d^{-}, u^{-}}$illustrated in Fig. 6. In fact, these systematic differences are especially marked for the $\left\langle x^{2}\right\rangle$ moments, as inspection of Fig. 6 attests. Especially notable in the right panel of Fig. 6 is the very strong sensitivity $\left|S_{f}\right| \gtrsim 0.75$ for a select subset of the gauge production data, particularly for $x \gtrsim 10^{-4}$ and separately for $x \gtrsim 0.1$. These especially strong constraints to $\left\langle x^{2}\right\rangle_{d^{-}}$originate from an amalgam of electroweak datasets, among which we find the $8 \mathrm{TeV}$ forward $W^{ \pm}, Z$ production cross section data of $\mathrm{LHCb}$ (LHCb8WZ'16), the analogous information at $7 \mathrm{TeV}$ (LHCb7ZWrap'15), as well as the forward-backward $e^{+} e^{-}$asymmetry in $W^{ \pm}, Z$ production at Runs 1 and 2 of CDF, CDF1Wasy'96 and CDF2Wasy'05. Compared with $\left\langle x^{2}\right\rangle_{u^{-}}$, on the other hand, for the second moment of $d^{-}(x)$ we find a substantially more restricted outlay of individual high-impact measurements in the fixed-target region, with significantly fewer data belonging to very high $x \gtrsim 0.4$ or $x \lesssim 0.2$ identified. Of these, the E605, NMCrat, and CCFR-F3 points enjoy special prominence. Many of these trends revealed by the sensitivity map in the right panel of Fig. 6 are further confirmed by quantitative ranking of the CTEQ-TEA experiments, especially based on the per-point sensitivities. For the second moment of the $d^{-}$distribution, the point-averaged sensitivity ranked experiments are D02Easy2'15 (0.519), CCFRF3'97 (0.381), LHCb7Wasy'12 (0.362), CMS7Masy2'14 (0.328), D02Masy'08 (0.293), CDF1Wasy'96 (0.252), LHCb8WZ'16 (0.217), LHCb7ZWrap'15 (0.214), and E605'91 (0.207).

For the aggregated sensitivities, here also we find the knowledge of the $d^{-}$second moment to be driven foremost by $x F_{3}$ data from CCFR and the combined HERA data, CCFR-F3'97 (32.8) and HERAI + II'15 (32.3), respectively. We note, however, that the total sensitivity of these leading experiments to the $d^{-}$distribution is reduced roughly $\sim 30 \%$ relatively to what was found for the corresponding $u$-quark sensitivities. Beyond these leading measurements, an assortment of $\mu$ and $\nu$ DIS and Drell-Yan experiments have the tightest pulls. Again in descending order, these are E605'91 (24.7), CDHSW-F3'91 (18.5), BCDMSd'90 (15.2), NMCrat'97 (14.8), BCDMSp'89 (14.4), E866pp'03 (13.8), and CDHSW-F2'91 (11.7).

\section{The gluon momentum fraction}

We can extend this program to the gluonic sector, considering the total nucleon momentum carried by gluons

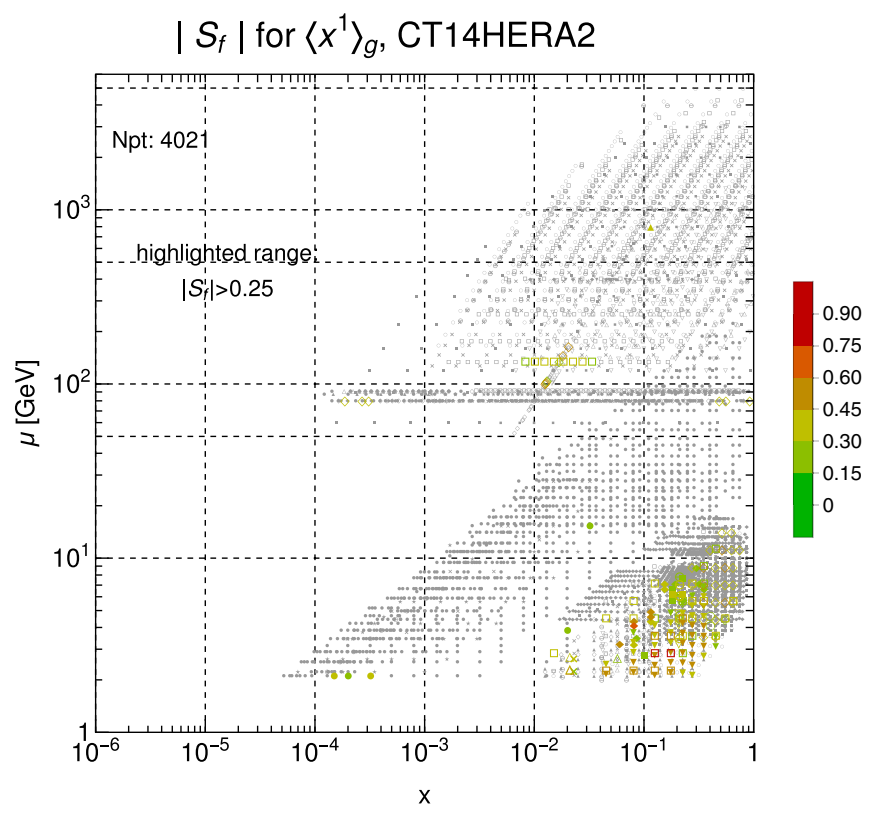

FIG. 7. Sensitivity of the CTEQ-TEA datasets to $\langle x\rangle_{g}$. The factorization scale at which the moment is evaluated is $\mu_{F}=2 \mathrm{GeV}$. Here we have only a single panel for $\langle x\rangle_{g}$, given that lattice computations thus far only exist for $\left\langle\mathcal{G}_{\mu \nu} \mathcal{G}^{\mu \nu}\right\rangle$.

as characterized by the first moment of the gluon distribution, $\langle x\rangle_{g}$; for the time being, this is the only moment of the gluon PDF which has been evaluated by multiple lattice groups, and we therefore concentrate on it primarily. Figure 7 illustrates the sensitivity to $\langle x\rangle_{g}$ of the CTEQTEA data considered in the plots of the preceding section.

From our analysis of the sensitivities, we arrive to perhaps not an entirely expected conclusion that a combination of DIS experiments holds the strongest cumulative sensitivity to $\langle x\rangle_{g}$, acquired through QCD radiative contributions at NLO and higher orders. Indeed, while neither the neutral-current DIS nor charged-current DIS probe the $g(x, Q)$ at the Born level, the degree of the Bjorken scaling violation in DIS cross sections at $x>0.1$ is known to be driven by the magnitude of the gluon PDF. Consequently the extensive DIS data at high $x$ provide the dominant constraints on $g(x, Q)$ in the $x$ region giving the largest contribution to $\langle x\rangle_{g}$.

In contrast, the hadron-hadron collider measurements such as production of inclusive jets or $t \bar{t}$ pairs, while probing the gluon PDFs already at the lowest order in $\alpha_{s}$, do not compete yet with DIS in their sensitivity to $\langle x\rangle_{g}$. Thus, unlike what was generally observed for the quark distribution moments reported above, only two experiments within the CTEQ-TEA set lie above the $\left\langle\left|S_{f}\right|\right\rangle>0.2$ ranking cut for $\langle x\rangle_{g}$. Based on their point-averaged sensitivities, these are both measurements of $F_{2}^{p}$ (albeit extracted from nuclear data), specifically, CDHSW-F2'91 (0.312) and CCFR-F2'01 (0.237). Immediately beyond these most valuable "per-point" measurements of $F_{2}$, several other 
experiments fall immediately below the cut with slightly weaker averaged sensitivities, including the $\nu$ DIS measurement of $F_{3}\left(x, Q^{2}\right)$ recorded by CCFR-F3'97 (0.188), the $7 \mathrm{TeV}$ ATLAS high- $p_{T} Z$ production data of ATL7ZpT'14 (0.184), and the $8 \mathrm{TeV} t \bar{t}$ measurements from ATLAS, ATL8ttb-mtt'16 (0.172).

Once again, in terms of the aggregated sensitivities, we observe a distinctly important role for the combined HERA dataset-HERAI + II'15 (49.2)—a result consistent with the significant precision and very wide coverage over $x$ and $Q^{2}$ of these cross section data. This wide coverage in $Q$ acts as a crucial lever arm to constrain the QCD evolution in the CT (or indeed any) parametrization, and thereby restricts the phenomenological behavior of the singlet and gluon distributions. After the reduced cross section measurements of HERA, a cascading series of nucleon or deuteron structure function $F_{2}^{p, d}$ measurements obtained on either hydrogen or nuclear targets contain the greatest share of information on the integrated gluon distribution. In descending order, these are the $\nu-\mathrm{Fe}$ DIS data of CDHSW-F2'91 (26.5), followed by $\mu$ scattering data from BCDMS, first on the deuteron, BCDMSd' 90 (25.8), as well as on a hydrogen target, BCDMSp'89 (24.9). Last, neutrino data from CCFR on $F_{2}$ [CCFR-F2'01 (16.3)] and $x F_{3}$ [CCFR-F3'97 (16.2)] have comparable pull between these two structure function measurement channels, and important influence in the wider fit. It is intriguing to notice that, while the aggregated pull of HERAI + II'15 (49.2) strongly dominates the spread of CTEQ-TEA experiments considered in isolation, were the leading $\nu-$ Fe experiments above regarded as a single experiment and their accumulated sensitivities simply combined directly, the result $(59 \gtrsim 49.2)$ surpasses the very large combined HERA dataset, which is based on $N_{p t}=1120$ cross section measurements. A similar observation holds for the BCDMS data. We therefore again stress the observation made above in the context of the aggregated CTEQ-TEA sensitivities to, e.g., $\left\langle x^{2}\right\rangle_{u^{-}-d^{-}}$: while the great extent of the combined HERA dataset's kinematical coverage frequently awards it a leading role in terms of its aggregated effect, agglomerations of much smaller, targeted datasets can have a comparable or greater combined effect, in principle.

Although they do not appear among the core of most decisive experiments detailed above, some of the newer LHC datasets canvassed in Ref. [22] are nonetheless among the top $\sim 10$ most sensitive experiments to $\langle x\rangle_{g}$ particularly the inclusive jet data found in Ref. [22] to provide important constraints to the gluon distribution overall. Specifically, these are the 8 and $7 \mathrm{TeV}$ CMS inclusive jet production data, CMS8jets'17 (7.1) and CMS7jets'14 (6.1), respectively. The future potential of the LHC jet data to constrain the gluon at large $x$ crucially depends on improvements in the understanding of significant systematic errors in these measurements.

\section{Flavor asymmetries of the nucleon sea}

As a final consideration in this section, we examine the sensitivities to linear combinations of the Mellin moments that quantify breaking of flavor $S U(3)$ symmetry of the nucleon's light-quark sea. The flavor structure of the proton's quark sea has for decades attracted sustained focus, especially regarding the dynamical origin of the observed charge-flavor asymmetry embodied by the breaking of the $\mathrm{SU}(3)_{\text {flavor }}$ PDF relation $\bar{u}(x)=\bar{d}(x)=s(x)=$ $\bar{s}(x)$ often assumed in the earliest phenomenological QCD global fits. Much formal interest in this topic attends to the fact that the $x$-dependent breaking of the $\mathrm{SU}(2)$ symmetry relation $\bar{d}(x, \mu)-\bar{u}(x, \mu)=0$ at low scales is principally understood as a feature of nonperturbative QCD [59,60]; for instance, patterns of dynamical chiral symmetry breaking in QCD favor hadronic dissociations of the proton having the form $p \rightarrow \pi^{+} n$ at low energies, which are thought to produce generic excesses of $\bar{d}$ over $\bar{u}$ in contributing to the nucleon's flavor structure [61-63]. It should be noted, however, that accounting for the detailed $x$ dependence of $\bar{d}(x)-\bar{u}(x)$ (or, equivalently, of deviations of the flavor ratio from $\bar{d} / \bar{u}=1$ ) in the context of meson-cloud models informed by this physical picture has been challenging.

Historically, much of the empirical information on parton-level flavor symmetry violation in the nucleon sea has been garnered through examinations of the unpolarized DIS structure functions. Formally, the structure functions can be described using well-established factorization theorems in terms of which they may be separated via convolution integrals over the long-distance PDFs and the perturbative coefficient functions.

In this context, a crucial observable first measured systematically by NMC $[64,65]$ is the Gottfried sum rule [66], which is sensitive to nonperturbative dynamics leading to the $\mathrm{SU}(2)$ flavor asymmetries in the light-quark sea mentioned above. The canonical expression of the sum rule can be obtained by applying the leading-order quark-parton model (QPM) to the isovector structure function difference,

$$
\begin{aligned}
\left.\int_{0}^{1} \frac{d x}{x}\left(F_{2}^{p}-F_{2}^{n}\right)\right|_{\mathrm{QPM}} & =\frac{1}{3} \int_{0}^{1} d x\left(u^{+}-d^{+}\right) \equiv \frac{1}{3}\langle 1\rangle_{u^{+}-d^{+}} \\
& =\frac{1}{3}-\frac{2}{3} \int_{0}^{1} d x(\bar{d}-\bar{u})
\end{aligned}
$$

where we have used isospin and the identities $q^{+}=q^{-}+2 \bar{q}$ and $\int d x\left(u^{-}-d^{-}\right)=1$ to rearrange the first line into the standard statement of the sum rule on the second. Most importantly, we highlight the fact that the zeroth moment of the isovector PDF, $\langle 1\rangle_{u^{+}-d^{+}}$[the right-hand side (RHS) of the first line of Eq. (22)], is directly related to the behavior of $\bar{d}-\bar{u}$, deviating from unity when $\langle 1\rangle_{\bar{d}-\bar{u}} \neq 0$. While this latter sea quark PDF moment appearing on the far RHS of Eq. (22) is not directly accessible on the lattice as a zeroth 
unpolarized moment, we are nonetheless able to compute the sensitivity of the CTEQ-TEA set to $\langle 1\rangle_{u^{+}-d^{+}}$and the related violation of the symmetric sea $\bar{u}=\bar{d}$ scenario formulated in terms of Mellin moments; this connection crucially motivates lattice measurement of the higher isovector moments $\left\langle x^{1,3}\right\rangle_{u^{+}-d^{+}}$treated in Secs. II D 1 and V, which would constrain the behavior of the phenomenological isovector distribution and inform its zeroth moment and analyses of the Gottfried sum rule. Moreover, the $x<0$ region of the isovector quasidistribution presented in Sec. III is immediately related to $\bar{d}(x)-\bar{u}(x)$, again implying a complementary avenue for lattice sensitivity to the light-quark sea.

In Fig. 8, we map the calculated sensitivities of the CTEQ-TEA high-energy dataset to the zeroth isovector moment $\langle 1\rangle_{u^{+}-d^{+}}$. While the general pattern of sensitivities in Fig. 8 is consistent with what we observed for the higher isovector moments illustrated in Fig. 3, the sensitivity is especially substantial here for $W^{ \pm}$and $Z$ production and the E866 cross section ratio (E866rat'01). To a lesser extent, we observe notable pulls from an assembly of fixed-target measurements, including several DIS experiments: the NMC structure function ratio information (NMCrat'97), the CCFR measurements of $x F_{3}^{p}$ (CCFR-F3'97), and the BCDMS $F_{2}^{p}$ data (BCDMSp'89).

These visible features of the PDFSense sensitivity map are largely borne out by the point-averaged CTEQ-TEA sensitivities to the zeroth isovector moment; as the

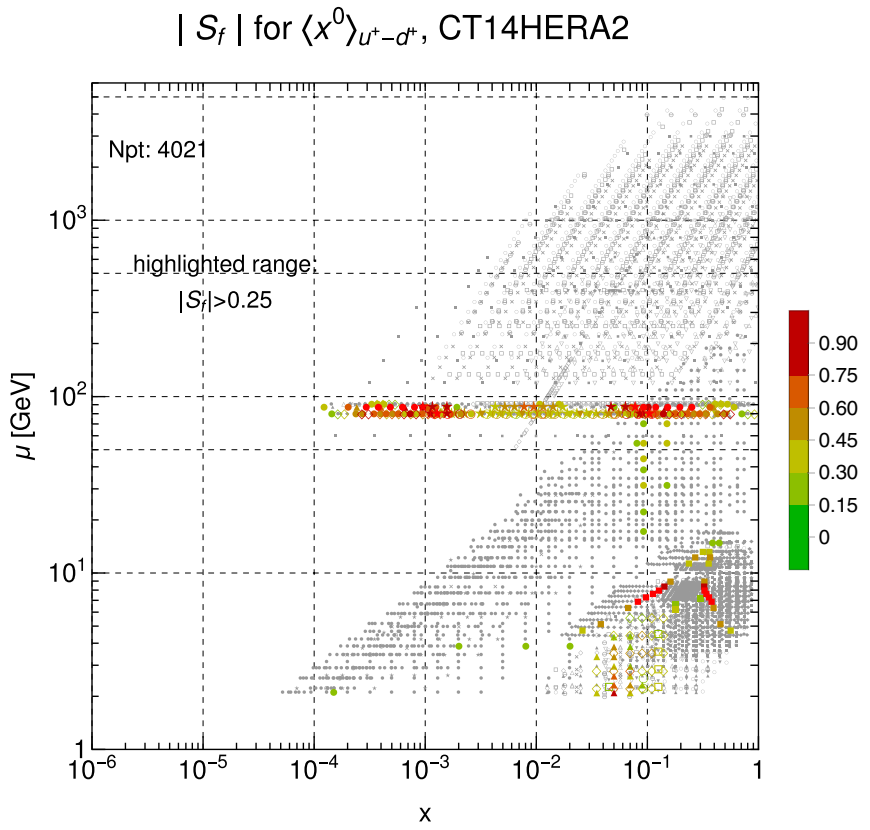

FIG. 8. Sensitivity of the CTEQ-TEA data to the first moment $\langle 1\rangle_{\left(u^{+}-d^{+}\right)}$. The factorization scale taken for the Mellin moment is $\mu=2 \mathrm{GeV}$. We stress that, while this combination is not directly calculable by the usual lattice methods, its appearance in the Gottfried sum rule motivates its study, as well as a focus upon higher moments. moments of the higher isovector moments and $d^{ \pm}$distributions explored above, the list of leading experiments ranked by this metric is again led by the $7 \mathrm{TeV} \mu$ asymmetry data recorded by CMS [CMS7Masy2'14 (0.645)], followed closely by the deuteron-proton cross section ratios measured by E866 [E866rat'01 (0.600)]; for the latter, this strong pull is notably consistent with E866's aim of probing the $x$ dependence of $\bar{d}(x) / \bar{u}(x)$ - a topic which continues to motivate modern experiments such as SeaQuest. Following these, the per-datum sensitivities of the CTEQ-TEA data are dominated by an amalgam of electroweak experiments represented by the rows of gauge boson data shown in Fig. 8. Again in descending order, these include LHCb7Wasy'12 (0.546), LHCb8WZ'16 (0.432), CMS7Easy'12 (0.381), CMS8Wasy'16 (0.370), LHCb7ZWrap'15 (0.351), D02Easy2'15 (0.323), D02Masy'08 (0.252), and ATL7WZ'12 (0.219).

Ordered according to their aggregated impact, on the other hand, only nine experiments exceed $\sum\left|S_{f}\right|>10$. These now include the usual DIS information from HERA and fixedtarget data from NMC, CCFR, and BCDMS, as well as the E866 $p p$ Drell-Yan cross section data-again owing to the aggregated pull of these enlarged datasets. In order of total sensitivity, these most decisive experiments are HERAI + II'15 (51.0), BCDMSp'89 (21.2), LHCb8WZ'16 (18.1), CCFR-F3'97 (15.7), NMCrat'97 (15.2), E866pp'03 (14.8), CMS8Wasy'16 (12.2), LHCb7ZWrap'15 (11.6), and BCDMSd'90 (10.2). Of these, there is again a pronounced effect from DIS experiments led by the combined HERA data which contribute by merit of their marginal per-datum sensitivity $\sim 0.05-0.1$ and the magnitude $N_{p t}$ of the datasets to which they belong, much as we observed for many of the other light-quark moments above.

\section{SENSITIVITIES TO QUARK QUASIDISTRIBUTIONS}

In addition to the PDF Mellin moments we analyzed in Sec. II, it has recently been proposed [20] that lattice QCD may evaluate parton "quasidistributions" over the quarkhadron longitudinal momentum fraction $x=k_{z} / P_{z}$ by evaluating matrix elements of the form

$\tilde{q}\left(x, P_{z}, \tilde{\mu}\right)=\int_{-\infty}^{\infty} \frac{d z}{4 \pi} e^{i x P_{z} z}\left\langle P\left|\bar{\psi}(z) \gamma^{z} U(z, 0) \psi(0)\right| P\right\rangle$,

where $U(z, 0)$ is a gauge link along the longitudinal $z$ direction and the argument $\tilde{\mu}$ represents the scales in the regularization-independent momentum subtraction scheme (RI-MOM) [67]; in practice, this involves the introduction of the parameters $p_{z}^{R}$ and $\mu_{R}$, which, for the purpose of this analysis, we fix to the values given in Ref. [52], $p_{z}^{R}=$ $2.2 \mathrm{GeV}$ and $\mu_{R}=3.7 \mathrm{GeV}$. Given its status as a matrix element of correlation functions along a spacelike longitudinal direction (unlike the ordinary $\overline{\mathrm{MS}} \mathrm{PDFs}$ ), the quasi- 

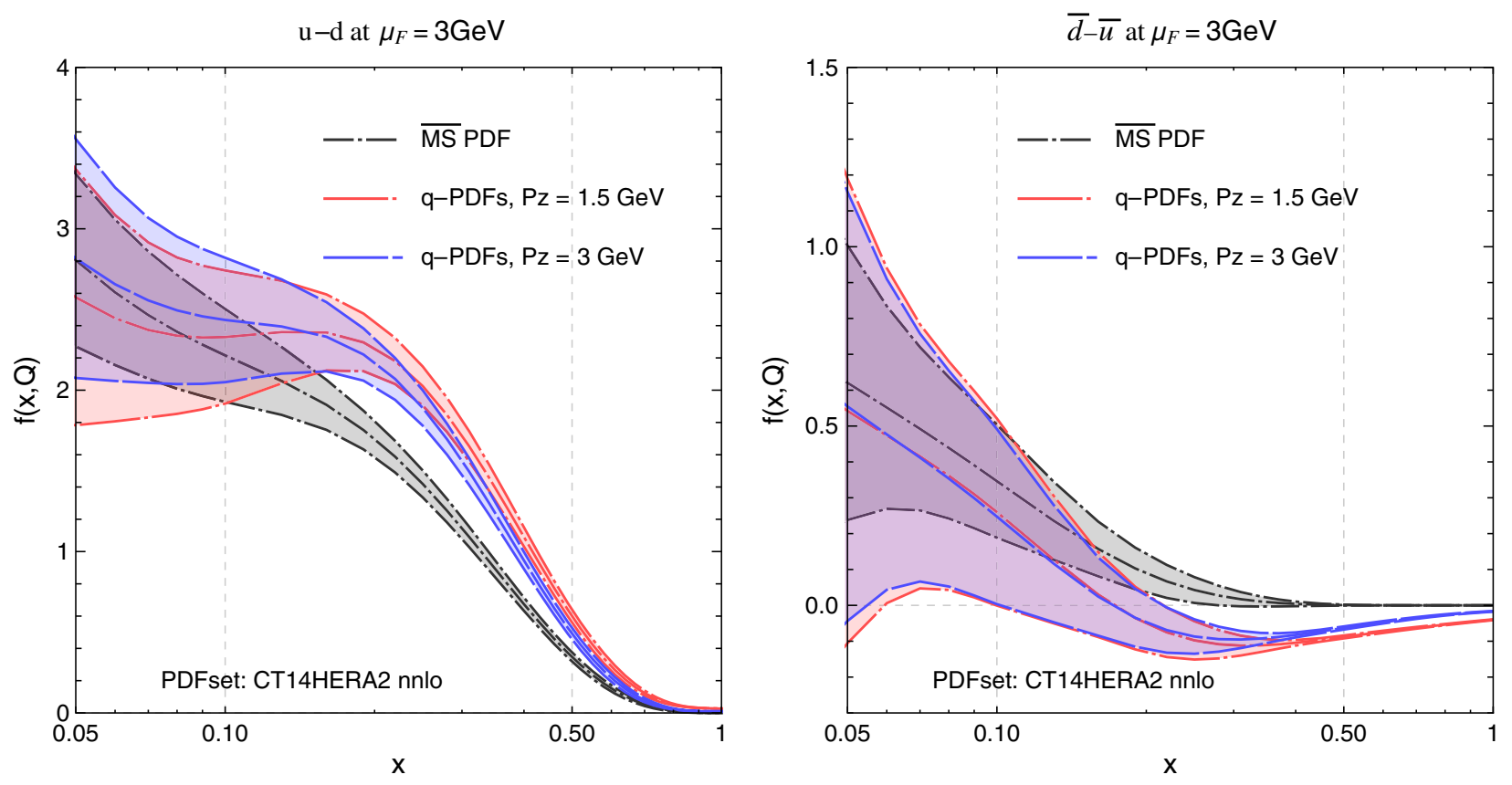

FIG. 9. The parton quasidistribution function $[\tilde{u}-\tilde{d}]\left(x, P_{z}, \tilde{\mu}\right)$ for $x>0$ (left panel) and $x<0$ (right panel), with the latter computed from the $\overline{\mathrm{MS}}$ PDF $\bar{u}-\bar{d}$ as given by CT14HERA2 NNLO.

distribution of Eq. (23) can be computed on the lattice ${ }^{3}$ and ultimately matched to the traditional phenomenological PDFs via an inversion of the expression given in Eq. (2). On the other hand, rather than inverting Eq. (2) to obtain the $\overline{\mathrm{MS}} \mathrm{PDF}$ from the lattice qPDF output, it is also possible to use Eq. (2) to compute the $P_{z}$-dependent qPDF from a phenomenological $\overline{\mathrm{MS}} \mathrm{PDF}$. Before lattice output matures to a sufficient level to help specify the $x$ dependence of PDFs through the combination of qPDF calculations and LaMET, it will be crucial to benchmark lattice calculations against knowledge derived from the fitted PDFs, and further improve perturbative matching and powercorrection formalism used to unfold PDFs from qPDF calculations on the lattice. Practically, these improvements are informed by direct comparisons of lattice-calculated qPDFs and those matched from phenomenological $\overline{\mathrm{MS}}$ PDFs computed according to Eq. (2). As such, if the PDF uncertainty of matched qPDFs determined from Eq. (2) could be further reduced by additional constraints from the appropriate experimental data, the ability to test and refine the LaMET formalism would be substantially enhanced. This logic extends especially to an understanding of the $P_{z}$ dependence of the matched qPDFs, which can be

\footnotetext{
${ }^{3}$ While the expression appearing in Eq. (23) is standard in the quasi-PDF literature, we clarify that in practice it can be advantageous to compute matrix elements with the replacement $\gamma^{z} \rightarrow \gamma^{t}$ [52]. While quasidistributions computed with $\gamma^{t}$ have similar limiting behavior for $P_{z} \rightarrow \infty$ as those determined using $\gamma^{z}$, lattice calculations carried out with $\gamma^{t}$ enjoy greater stability against operator mixing [68], and the numerical results shown in this section therefore assume this procedure.
}

particularly sensitive to power corrections and the perturbative order of the matching formalism.

Thus, to illustrate the current knowledge of qPDFs predicted using Eq. (2), Fig. 9 displays the qPDFs derived from the CT14HERA2 NNLO PDFs for $P_{z}=1.5$ and $3 \mathrm{GeV}$, together with the bands of current uncertainties on these qPDFs estimated at the $90 \%$ probability level. The uncertainties are computed according to the Hessian master formula (16) from CT14HERA2 NNLO error sets. The error bands for the collinear CT14HERA2 NNLO PDFs are also shown, labeled as "MS PDFs." In fact, a Hessian error set for the qPDFs at a given $P_{z}, \tilde{\mu}$, and $\mu$ can be obtained by applying Eq. (2) to each error set of the CT14HERA2 NNLO ensemble. This algorithm is entirely analogous to the calculation of the error ensemble for the Mellin moments using Eq. (12). The qPDF Hessian set $\tilde{q}_{j \in\{2 N\}}\left(x, P_{z}, \tilde{\mu}\right)$ may then be used to compute the sensitivities of the CTEQ-TEA set to the quark quasidistributions along the lines described in Sec. II B.

\section{A. Sensitivity maps}

For this purpose, we again deploy PDFSense, this time to study in a proof-of-principle demonstration showing the constraints from the present data on the $P_{z}$-dependent quasidistributions computed according to Eq. (2) from the underlying phenomenological PDFs, given the current knowledge of the perturbative matching coefficient $Z$ in Eq. (23), computed using the one-loop formalism of Ref. [52]. In the present section, we assume an $\overline{\mathrm{MS}}$ factorization scale of $\mu_{F}=3 \mathrm{GeV}$, as in Ref. [52]. 
We wish to highlight both the dependence upon $x$ and $P_{z}$ of the quasidistribution of the CTEQ-TEA sensitivities, and we therefore plot in this section four panels in Fig. 10 showing the behavior of the quasidistribution $[\tilde{u}-\tilde{d}]\left(x, P_{z}, \tilde{\mu}\right)$ at two representative values at relatively large $|x|: x=-0.5,0.85$ for $P_{z}=1.5$ and $3 \mathrm{GeV}$. For the quasidistributions evaluated for $x<0$, we note the implementation of the canonical

\section{$\left|S_{f}\right|$ for $[\tilde{u}-\tilde{d}]\left(\mathrm{x}=-0.5, P_{z}=3 \mathrm{GeV}\right), \mathrm{CT} 14 \mathrm{HERA} 2$}

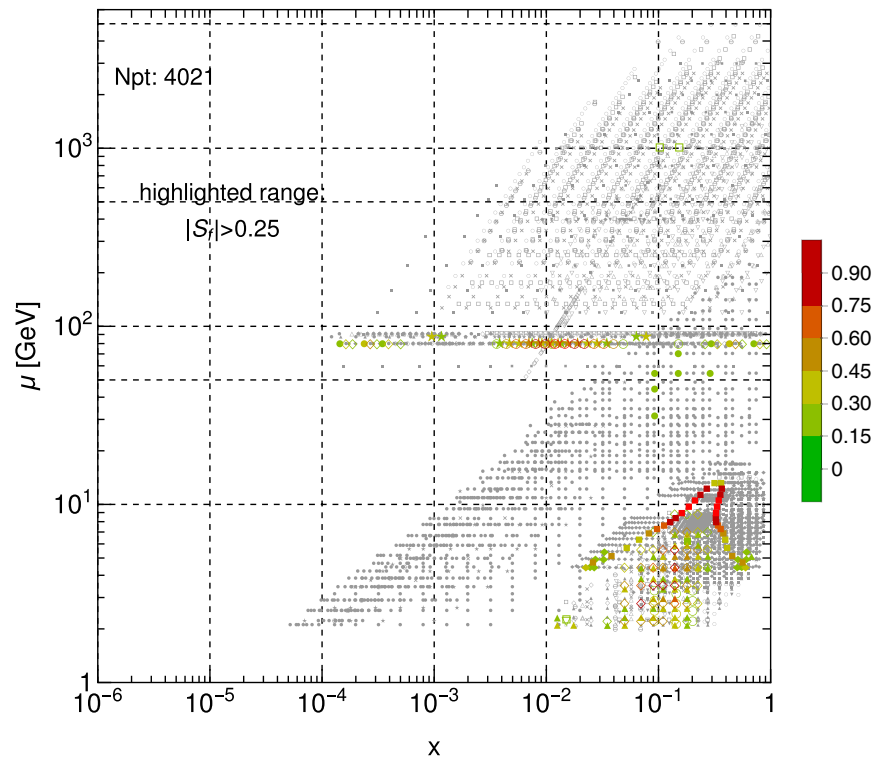

(a) Sensitivity to $[\widetilde{u}-\widetilde{d}]\left(x=-0.5, P_{z}=3.0 \mathrm{GeV}\right)$.

\section{$\left|S_{f}\right|$ for $[\tilde{u}-\tilde{d}]\left(\mathrm{x}=-0.5, P_{z}=1.5 \mathrm{GeV}\right), \mathrm{CT} 14 \mathrm{HERA} 2$}

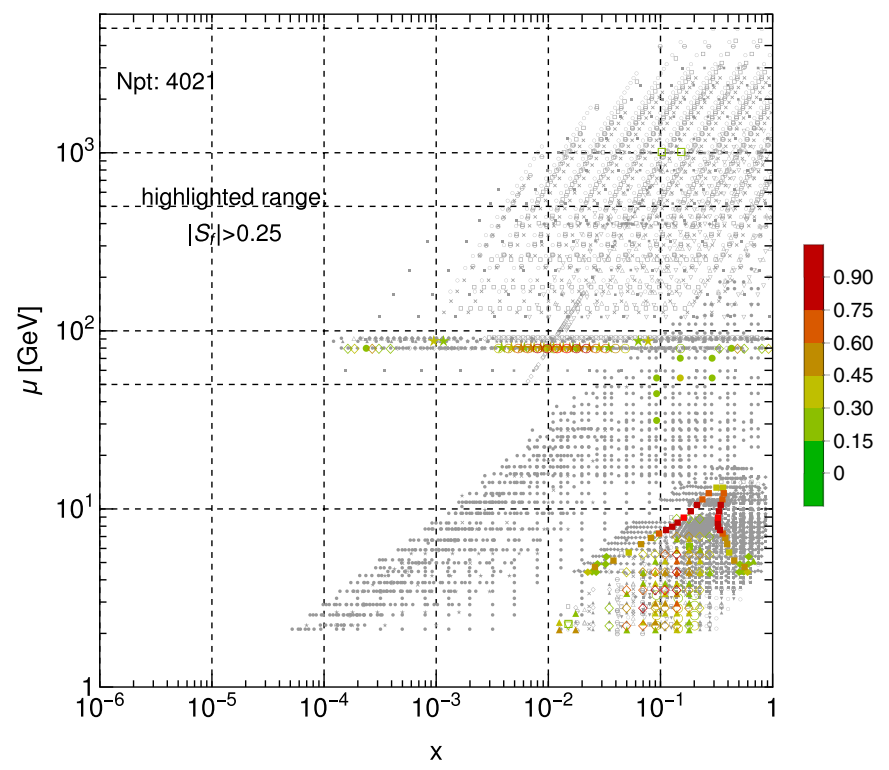

(c) Sensitivity to $[\widetilde{u}-\widetilde{d}]\left(x=-0.5, P_{z}=1.5 \mathrm{GeV}\right)$. relation $\bar{q}(x)=-q(-x)$, such that the negative $x$ region of the quasidistribution is related to the $x$ dependence of the phenomenological antiquark PDFs (with the logic that backward-moving quarks with longitudinal momenta $k_{z}=$ $x P_{z}<0$ are identifiable with forward-moving antiquarks).

The essential point that emerges from Fig. 10 is the fact that a common cluster of experiments, mostly of higher $x$

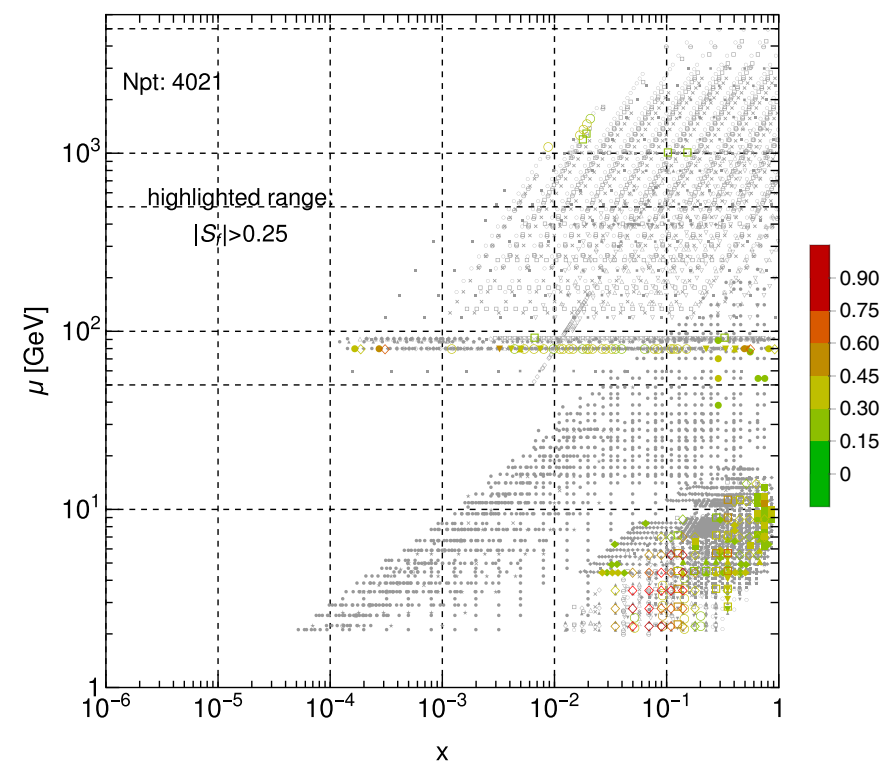

(b) Sensitivity to $[\widetilde{u}-\widetilde{d}]\left(x=0.85, P_{z}=3.0 \mathrm{GeV}\right)$.

\section{$\left|S_{f}\right|$ for $[\tilde{u}-\tilde{d}]\left(\mathrm{x}=0.85, P_{z}=1.5 \mathrm{GeV}\right), \mathrm{CT} 14 \mathrm{HERA} 2$}

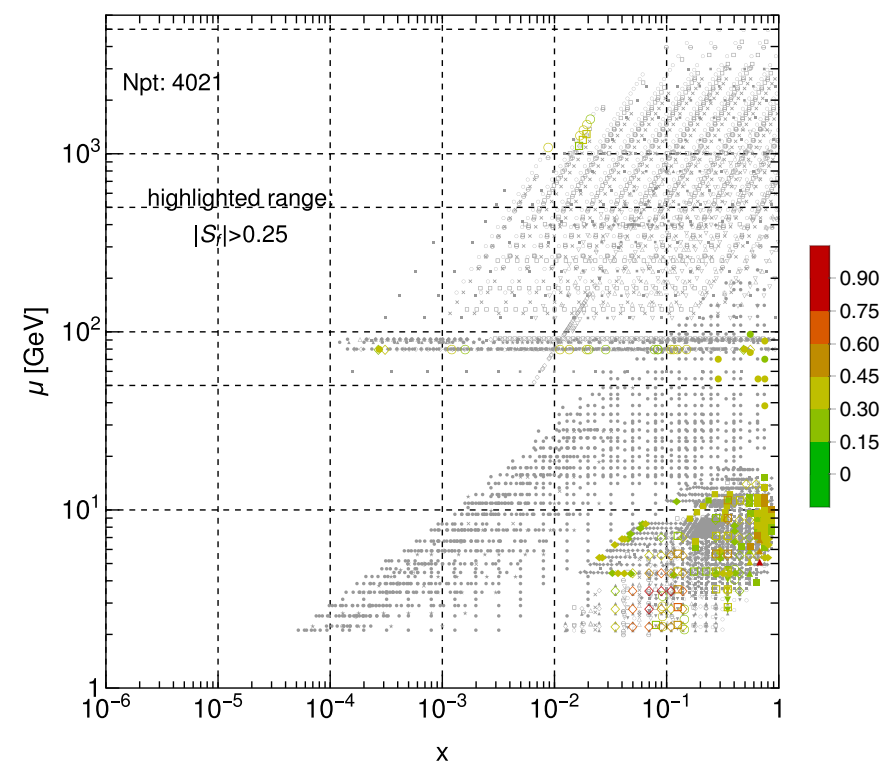

(d) Sensitivity to $[\widetilde{u}-\widetilde{d}]\left(x=0.85, P_{z}=1.5 \mathrm{GeV}\right)$.

FIG. 10. The isovector quark quasidistribution at large values of $\pm x=k_{z} / P_{z}$, i.e., $x=-0.5$ (left panels) and $x=0.85$ (right panels) for a relatively fast moving proton, boosted to $P_{z}=3 \mathrm{GeV}$ (top panels), and comparatively slow protons boosted to $1 / 2$ this momentum, $P_{z}=1.5 \mathrm{GeV}$ (lower panels). 
fixed-target and $W^{ \pm}$production and asymmetry measurements, represents the primary constraint to the $\bar{u}-\bar{d}$ quasidistribution in a fashion that is largely independent of the boosted hadron's momentum $P_{z}$. Some intriguing $P_{z}$ dependence does begin to emerge, however, for the CTEQTEA sensitivities to the highest $x$ region of the isovector quasidistribution, evident in Fig. 10 by comparing the $x=0.85$ maps obtained for $P_{z}=3.0$ and $1.5 \mathrm{GeV}$ in the upper-right (b) and lower-right (d) panels. In particular, the $P_{z}$ dependence appearing in the $\left|S_{f}\right|(x, \mu)$ distributions of Fig. 10 is signaled by the enhancement in the sensitivity to $[\tilde{u}-\tilde{d}](x=0.85)$ of the highest $x \gtrsim 0.5 \mu p$ DIS points of BCDMSp' 89 and NMCrat'97 found for the $P_{z}=$ $1.5 \mathrm{GeV}$ [Fig. 10(d)] compared to the analogous calculation, for the sensitivities to the $P_{z}=3 \mathrm{GeV}$ quasidistribution [Fig. 10(b)]. This relative increase of the sensitivity of the high- $x$ DIS information is offset by an accompanying relative reduction in the general sensitivity of the $W^{ \pm}, Z$ production data, which for $P_{z}=3 \mathrm{GeV}$ exhibited significant pulls on $[\tilde{u}-\tilde{d}](x=0.85)$, especially for the $7 \mathrm{TeV} A_{\mu}(\eta)$ asymmetry data taken by CMS, CMS7Masy2'14. The implication of these observations is the fact that a careful exploration of the nucleon structure function at high $x$ may provide crucial information for constraining the $P_{z}$ dependence of the quasidistributions required for a robust application of LaMET. The qPDFs do not have the usual $x \in[0,1]$ support of the light-front PDFs and are finite at $x>1$. For $P_{z}=1.5,3 \mathrm{GeV}$, the isovector
qPDFs considered in this analysis are already rapidly vanishing in the $x>1$ region, however, and the sensitivity maps for $[\tilde{u}-\tilde{d}](x \gtrsim 1)$ are qualitatively similar to those shown in Figs. 10(b) and (d).

In addition, one can examine the qPDF sensitivity for shallower values of $|x| \sim 0$, which we illustrate in Fig. 11 to further explicate the sensitivity dependence on $x$ of the qPDF. In Fig. 11, we plot $[\tilde{u}-\tilde{d}](x=0 .-05)$ (a) and $[\tilde{u}-\tilde{d}](x=0.05)(\mathrm{b})$, both for $P_{z}=1.5 \mathrm{GeV}$. Compared with $[\tilde{u}-\tilde{d}](x=-0.5)$ shown in Fig. 10(c), we find a significant enhancement in the sensitivity of the smaller- $x$ fixed-target and E866 ratio data to $[\tilde{u}-\tilde{d}](x=-0.05)$ in Fig. 11(a); this shift is accompanied by a moderate redistribution in the sensitivity of the $W$-production data about $x \sim 10^{-2}$ in $(x, \mu)$ space. On the other hand, in moving from $x=-0.05 \rightarrow 0.05$, changes to the data pulls on $[\tilde{u}-\tilde{d}](x=0.05)$ plotted in Fig. 11(b) occur mostly for the $W$ - and $Z$-production data, especially at larger rapidities, corresponding to $x \gtrsim 10^{-1}$ and $x \lesssim 10^{-3}$, while the sensitivities of the data in the fixed-target region show comparatively weaker dependence on $x$ of the qPDF in the $|x| \sim 0$ region. The qPDF sensitivities of the full CTEQ-TEA data therefore exhibit a complex dependence upon $x$ of the qPDF that implies the importance of experimental information from diverse channels in improving phenomenological benchmarks for future qPDF lattice calculations.

\section{$\left|S_{f}\right|$ for $[\tilde{u}-\tilde{d}]\left(\mathrm{x}=-0.05, P_{z}=1.5 \mathrm{GeV}\right)$, CT14HERA2}

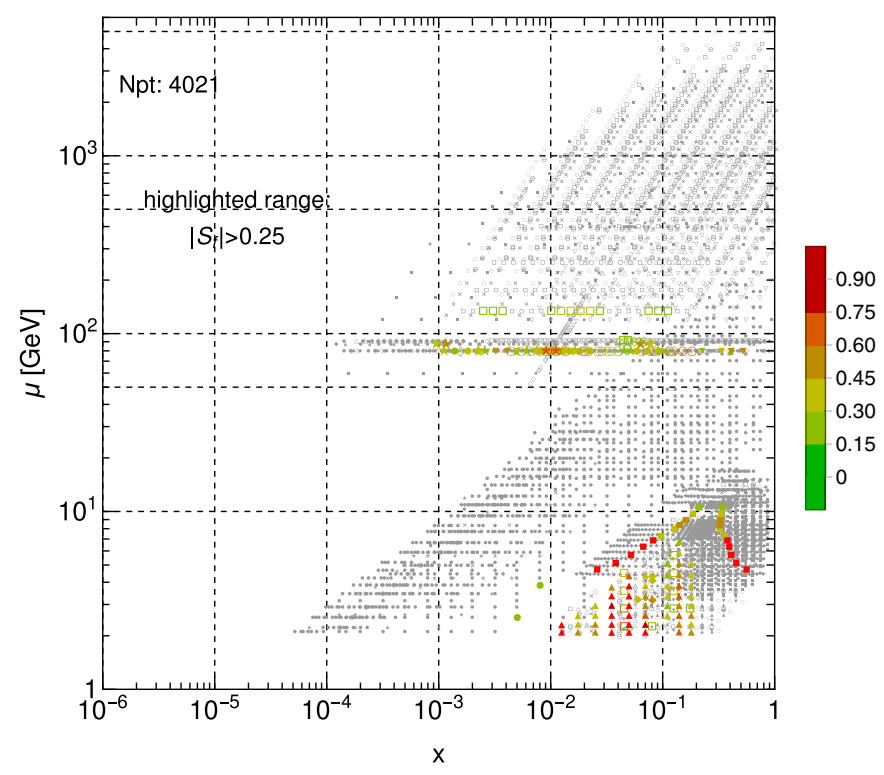

(a) Sensitivity to $[\widetilde{u}-\widetilde{d}]\left(x=-0.05, P_{z}=1.5 \mathrm{GeV}\right)$.

\section{$\left|S_{f}\right|$ for $[\tilde{u}-\tilde{d}]\left(\mathrm{x}=0.05, P_{z}=1.5 \mathrm{GeV}\right), \mathrm{CT} 14 \mathrm{HERA} 2$}

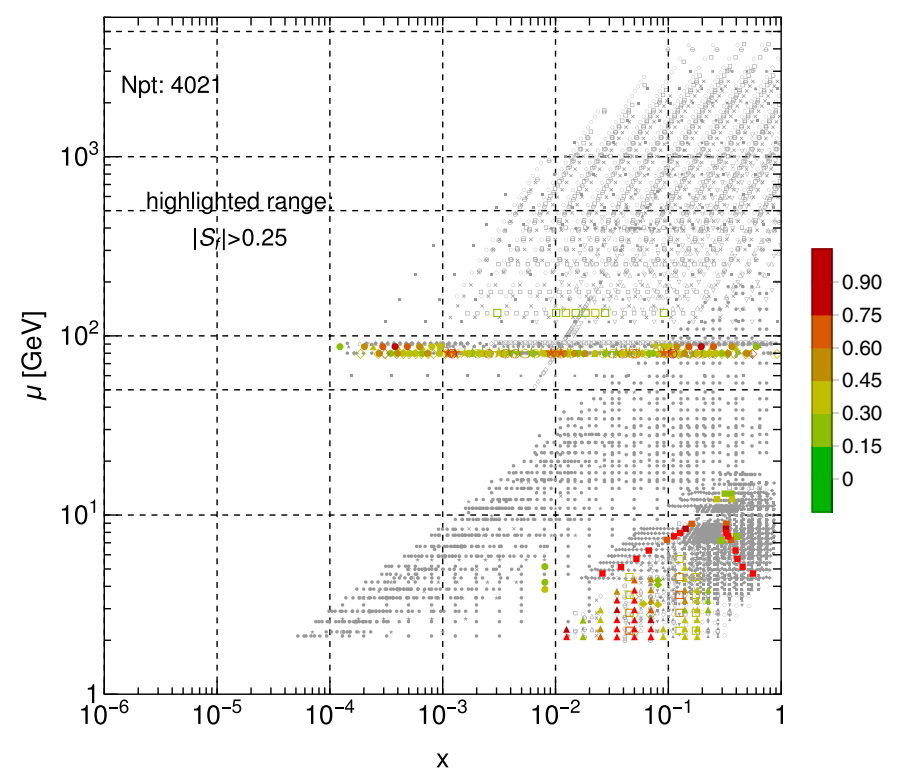

(b) Sensitivity to $[\widetilde{u}-\widetilde{d}]\left(x=0.05, P_{z}=1.5 \mathrm{GeV}\right)$.

FIG. 11. As Fig. 10 for the isovector quark quasidistribution, but now for comparatively small values of $|x|$, in this case, $x=-0.05$ (left panel) and $x=0.05$ (right panel). Here, we plot $\left|S_{f}\right|$ maps only for the smaller boost scale, $P_{z}=1.5 \mathrm{GeV}$, as we find the $P_{z}$ dependence of the sensitivities at these smaller values of $|x|$ to be very mild. 


\section{MOTIVATION FOR FUTURE EXPERIMENTS}

A number of futuristic machines have been either proposed or planned with a stated aim (among other physics motivations) of disentangling the collinear structure of hadronic states, including various futuristic hadroncollider experiments such as the HL-LHC [69] and, e.g., the AFTER@CERN proposal [70]. Among these proposals, a number of lepton-hadron colliders have been advocated, especially a future U.S.-based EIC [71-74] and a lepton-nucleon/nucleus variant of the LHC, the Large Hadron-Electron Collider (LHeC) [75]. An EIC, in particular, is most likely to serve the dedicated role of a hadron tomography machine, given its high-luminosity coverage of the crucial few-GeV quark-hadron transition region in the kinematical parameter space. An EIC would enjoy unprecedented facility in unfolding the nucleon's collinear and transverse structure at scales adjacent to the nucleon mass, $\gtrsim M$. The science output of an EIC would greatly build upon the JLab12 program [76], while the kinematic coverage of an EIC would be particularly favorable for constraining the quantities accessible in next-generation lattice QCD calculations.

As a simple illustration of the potential of a future DIS program, we compute the sensitivity maps that result from implementing a set of pseudodata into the PDFSense framework and examining our impact metrics for the first moment of the isovector distribution $\langle x\rangle_{u^{+}-d^{+}}$and the high- $x$ behavior of the $P_{z}=1.5 \mathrm{GeV}$ isovector quark

\section{$\left|S_{f}\right|$ for $\left\langle x^{1}\right\rangle_{u^{+}-d^{+}}$, CT14HERA2}

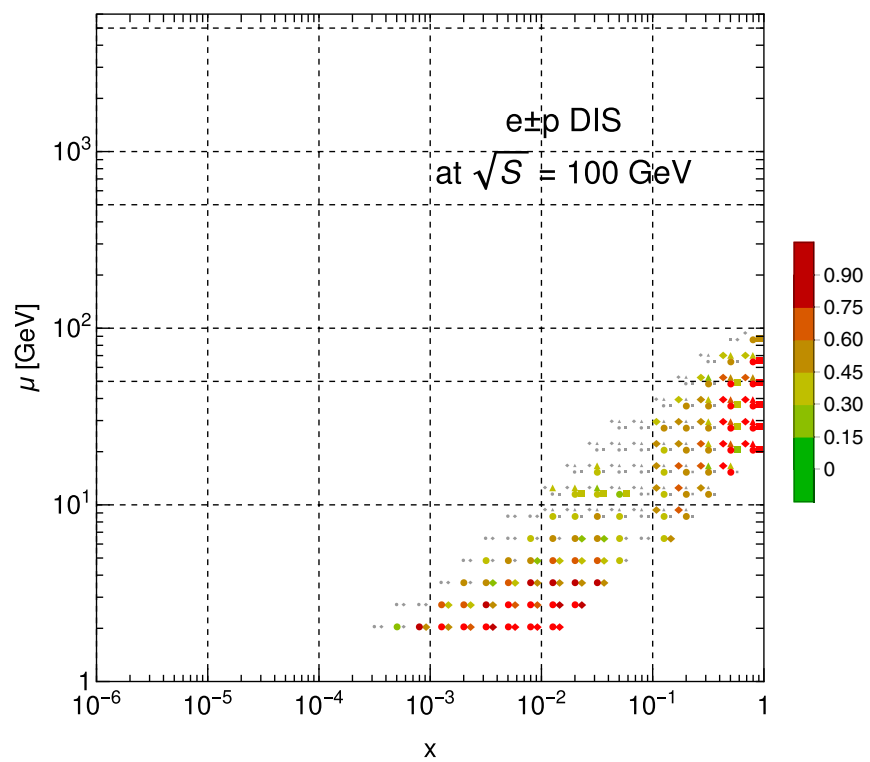

quasidistribution $[\tilde{u}-\tilde{d}]\left(x, P_{z}, \tilde{\mu}\right)$. To avoid marrying our predictions to the specifics of a particular experimental proposal, we consider an archetypal machine that measures the reduced cross section $\sigma_{r}\left(x, Q^{2}\right)$ via inclusive $e^{ \pm}$scattering on an unpolarized proton target. For this example, cross section pseudodata are produced in bins specified by a realistic Monte Carlo event generator about the CT14HERA2 NNLO theoretical prediction with a Gaussian smearing function of standard deviation equal to an assumed uncorrelated error taken from the Monte Carlo simulation. Theoretical predictions are for the reduced cross section measured in $e^{ \pm} p$ scattering at $\sqrt{s}=100 \mathrm{GeV}$ in both neutral- and charge-current interactions. For this illustration, statistical uncertainties are based upon assumed integrated luminosities of $\mathcal{L}=100 \mathrm{fb}^{-1}$ in $e^{-} p$ scattering and $\mathcal{L}=$ $10 \mathrm{fb}^{-1}$ for $e^{+} p$ events.

Figure 12 estimates the potential impact such a leptonnucleon collider might have on the above-noted lattice computable quantities: in the left panel, the first moment, $\langle x\rangle_{u^{+}-d^{+}}$, of the isovector quark distribution, and, in the right panel, the large- $x$ quasi-PDF matched from the CT14HERA2 NNLO NNLO PDF set according to Eq. (2). In both panels, physical channels for the inclusive DIS process are explicitly represented by unique symbols; these are $\mathrm{NC} e^{-} p$ (disks); $\mathrm{NC} e^{+} p$ (diamonds); $\mathrm{CC} e^{-} p$ (squares); $\mathrm{CC} e^{+} p$ (triangles).

Figure 12 indicates that measurements at a high-luminosity lepton-nucleon collider can considerably improve

\section{$\left|S_{f}\right|$ for $[\tilde{u}-\tilde{d}]\left(\mathrm{x}=0.85, P_{z}=1.5 \mathrm{GeV}\right)$, CT14HERA2}

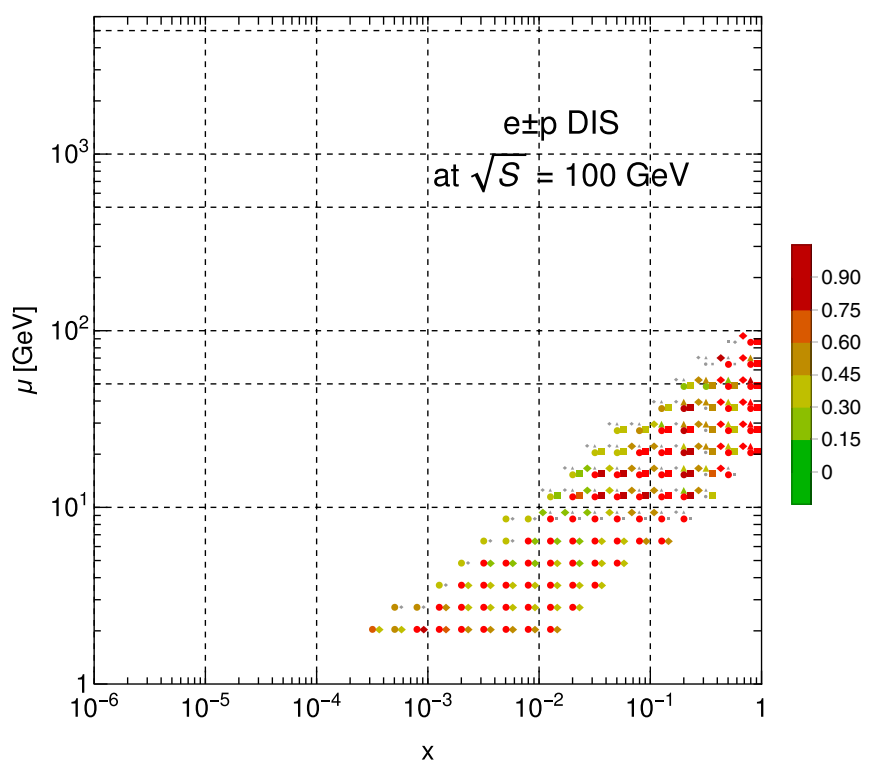

FIG. 12. Sensitivity of pseudodata for the inclusive DIS of $e^{ \pm}$on unpolarized protons at $\sqrt{s}=100 \mathrm{GeV}$ to the first Mellin moment of the isovector PDF combination $\langle x\rangle_{u^{+}-d^{+}}$(left) at an $\overline{\mathrm{MS}}$ scale of $\mu=2 \mathrm{GeV}$. The right panel shows the sensitivity to the high- $x$ behavior of the quasidistribution for the same isovector PDF, $[\tilde{u}-\tilde{d}]\left(x, P_{z}, \tilde{\mu}\right)$ for $P_{z}=1.5 \mathrm{GeV}, \mu=3 \mathrm{GeV}$, and $\tilde{\mu}$ taken from Ref. [52] computed according to Eq. (2). The plotted symbols characterize the specific channel as NC $e^{-} p$ (disks); NC $e^{+} p$ (diamonds); $\mathrm{CC} e^{-} p$ (squares); $\mathrm{CC} e^{+} p$ (triangles). 


\section{CT14HERA2 NNLO, Mellin moments, Sensitivity per data point $\langle|S|\rangle$}

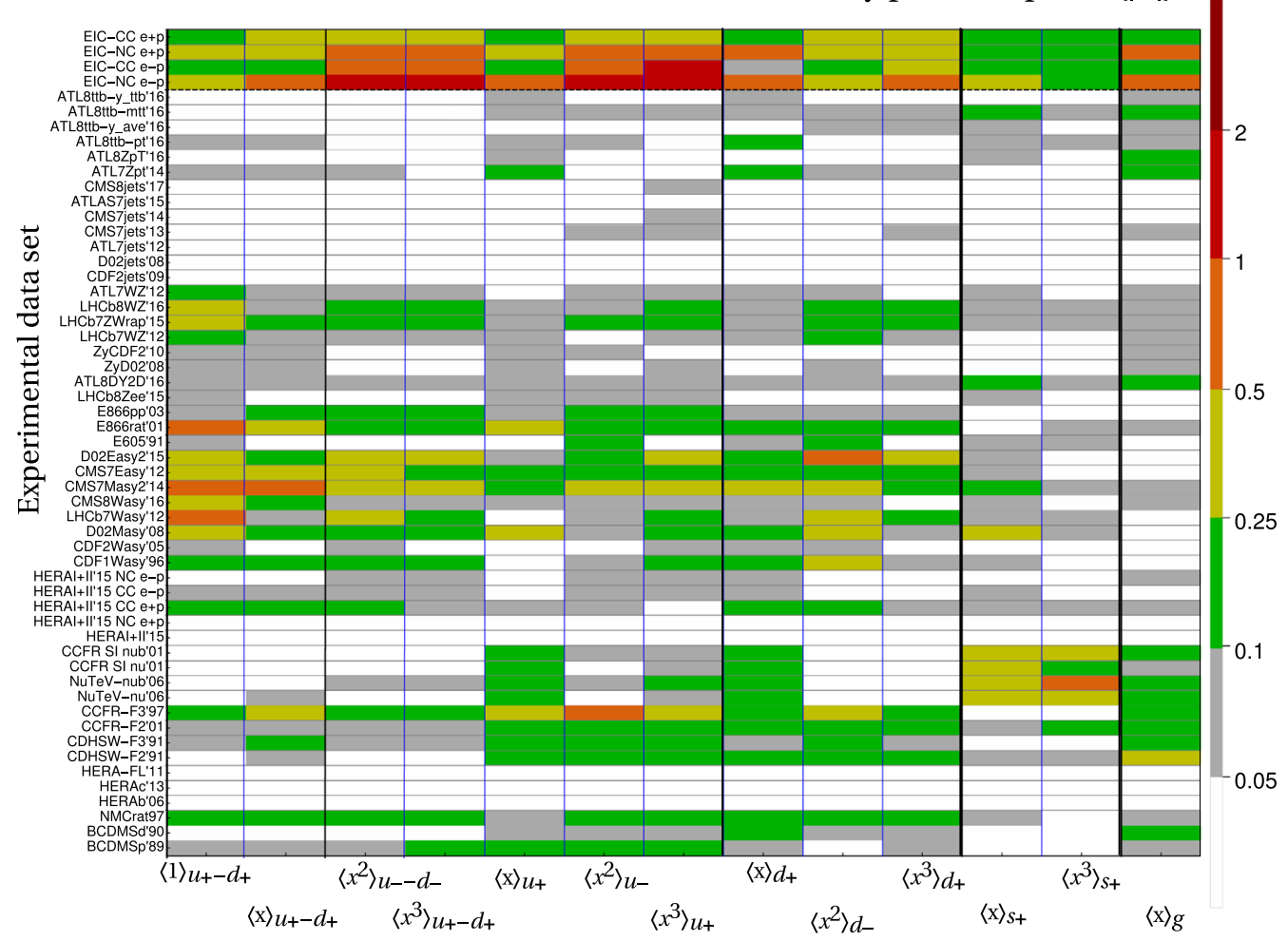

CT14HERA2 NNLO, Mellin moments, Total sensitivity $\Sigma|S|$

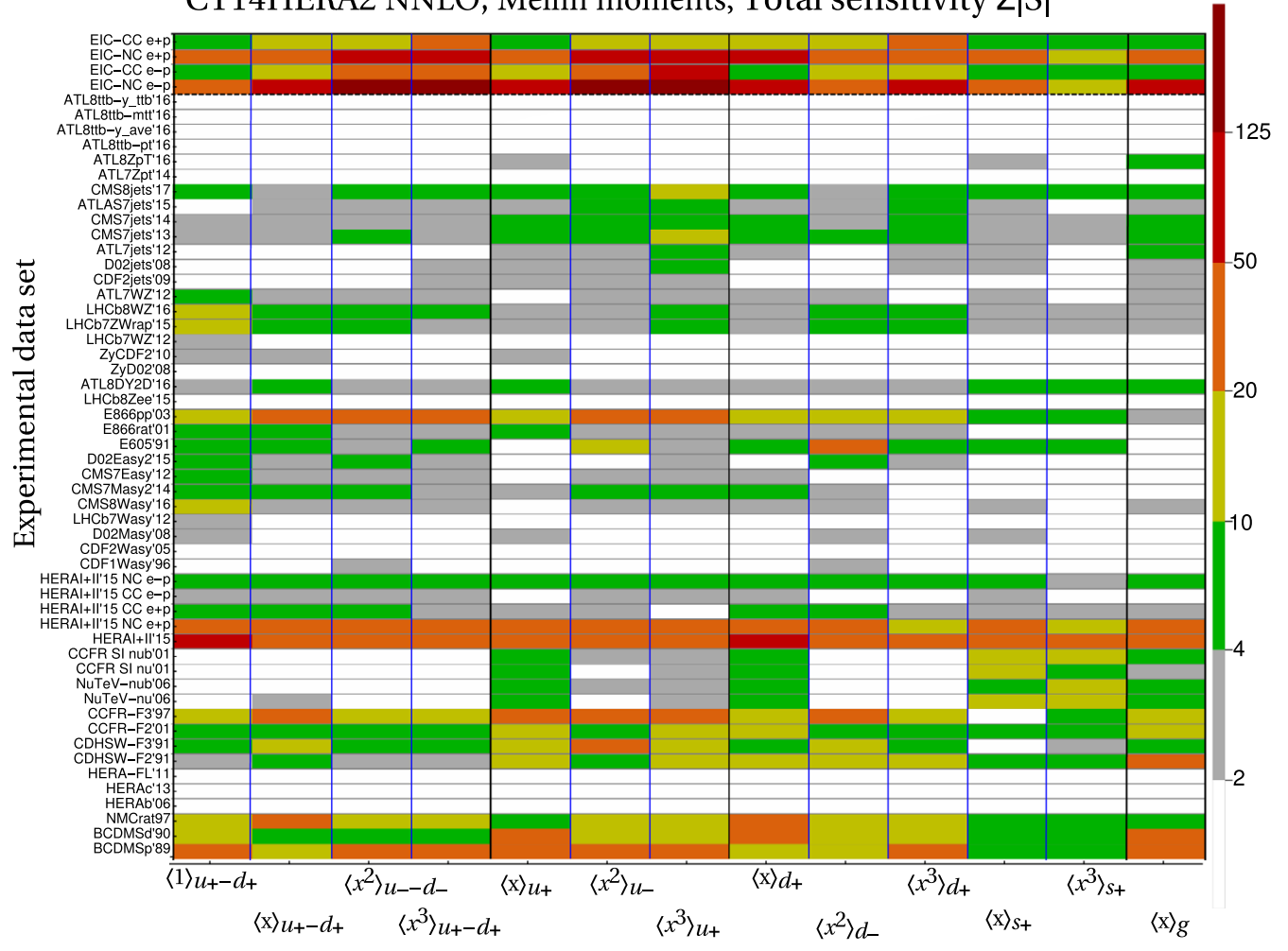

FIG. 13. A graphical representation of the sensitivity of each of the constituent experiments contributing to the CTEQ-TEA dataset. The grids summarize the point-averaged (upper panel) and summed or total (lower panel) sensitivities of each experimental dataset to each moment for several flavor combinations of strong interest; the color of the cell encodes the magnitude of the combined sensitivity for that particular moment. In addition, we also include in the uppermost rows the sensitivities obtained for pseudodata consistent with a future EIC-like lepton-nucleon collider experiment in the inclusive, unpolarized sector. 

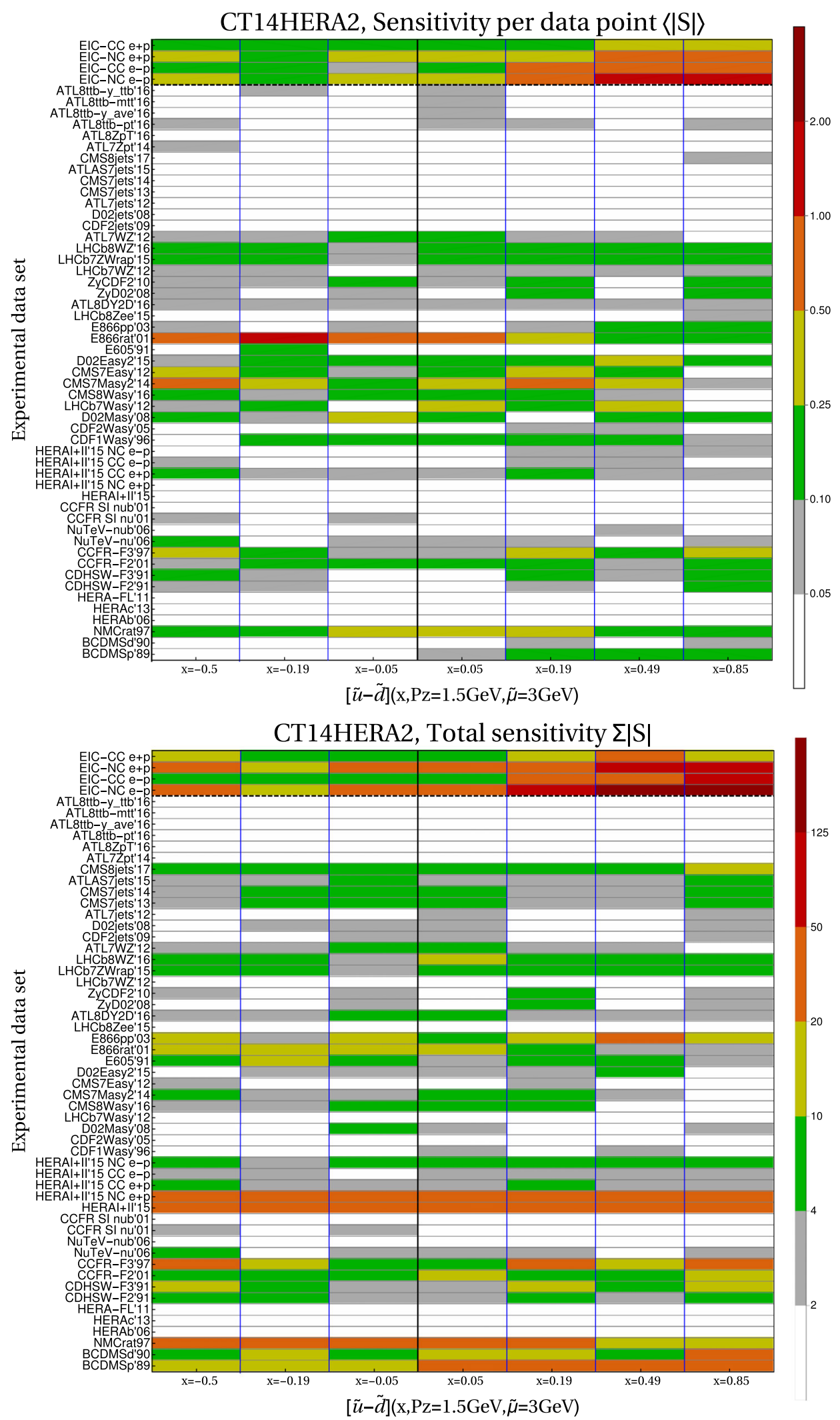

FIG. 14. As Fig. 13, but in this case illustrating the per-datum (upper panel) and aggregated (lower panel) sensitivities of the experiments within the CTEQ-TEA set to specific $x$ regions (indicated at the bottom) of the isovector quasidistribution at $P_{z}=1.5 \mathrm{GeV}$. As in Fig. 13, we again show the evaluations for pseudodata corresponding to a future EIC-like machine at the top. 
the constraints on both quantities considered. In particular, they supply very substantial sensitivities across the range of $x$ of the dataset, with especially large predicted impacts for $x \gtrsim 0.1$ as well as the $x \lesssim 0.01$ regions. A notable feature of this information is the separation that emerges illustrating the crucial role of both electron and positron probes: once separated among channels, a prominent effect is the important role of the CC-mediated positron-nucleon scattering $\left(e^{+} p\right)$; this impact is very pronounced at large $x \gtrsim 0.1$. At the EIC, the sensitivities at $x>0.01$ arise from both the NC and CC channels, while at $x<0.001$, the sensitivities mainly come from $\mathrm{NC} e^{+} p$ and $e^{-} p$ channels.
The $\left|S_{f}\right|\left(x_{i}, \mu_{i}\right)$ sensitivity maps appearing throughout this analysis, including those in Fig. 12 for the EIC pseudodata, are complemented by the companion plots shown in Figs. 13 and 14. These figures integrate the information displayed in the sensitivity maps for the PDF moments (Fig. 13) and qPDFs (Fig. 14) experiment-byexperiment, thereby summarizing the point-averaged sensitivities $\left\langle\left|S_{f}\right|\right\rangle$ for all of the CTEQ-TEA experiments and EIC pseudodata considered in this analysis, as well as the corresponding aggregated sensitivities, $\sum_{i \in N_{p t}}\left|S_{f}^{i}\right|$. These companion plots encapsulate the related information summarized in Tables II-IV of Appendix. These tables sort the

TABLE II. Experimental datasets considered as part of CT14HERA2 NNLO and included in this analysis: deep-inelastic scattering. We point out that the numbering scheme (Expt ID) included in this and subsequent tables follows the standard CTEQ labeling system with, e.g., Expt. IDs of the form 1XX representing DIS experiments. The HERA combined dataset HERAI + II'15 consists of both neutral-current (NC) and charge-current (CC) scattering events.

\begin{tabular}{lcccc}
\hline \hline Experiment name & Expt ID & Dataset details & $N_{p t}$ \\
\hline BCDMSp'99 & 101 & BCDMS $F_{2}^{p}$ & {$[77]$} & 337 \\
BCDMSd'90 & 102 & BCDMS F & {$[7]$} & 250 \\
NMCrat'97 & 104 & NMC $F_{2}^{d} / F_{2}^{p}$ & {$[79]$} & 123 \\
CDHSW-F2'91 & 108 & CDHSW $F_{2}^{p}$ & {$[80]$} & 85 \\
CDHSW-F3'91 & 109 & CDHSW $F_{3}^{p}$ & {$[80]$} & 96 \\
CCFR-F2'01 & 110 & CCFR $F_{2}^{p}$ & {$[81]$} & 69 \\
CCFR-F3'97 & 111 & CCFR $x F_{3}^{p}$ & {$[82]$} & 86 \\
NuTeV-nu'06 & 124 & NuTeV $\nu \mu \mu$ SIDIS & {$[83]$} & 38 \\
NuTeV-nub'06 & 125 & NuTeV $\bar{\nu} \mu \mu$ SIDIS & {$[83]$} & 33 \\
CCFR SI nu'01 & 126 & CCFR $\nu \mu \mu$ SIDIS & {$[84]$} & 40 \\
CCFR SI nub'01 & 127 & CCFR $\bar{\nu} \mu \mu$ SIDIS & {$[84]$} & 38 \\
HERAb'06 & 145 & H1 $\sigma_{r}^{b}\left(57.4 \mathrm{pb}^{-1}\right)$ & {$[85,86]$} & 10 \\
HERAc'13 & 147 & Combined HERA charm production $\left(1.504 \mathrm{fb}^{-1}\right)$ & {$[87]$} & 47 \\
HERAI + II'15 & 160 & HERA1 +2 Combined NC and CC DIS $\left(1 \mathrm{fb}^{-1}\right)$ & {$[88]$} & 1120 \\
HERA-FL'11 & H1 $F_{L}\left(121.6 \mathrm{pb}^{-1}\right)$ & {$[89]$} & 9 \\
\hline \hline
\end{tabular}

TABLE III. Same as Table II, showing experimental data sets for production of vector bosons, single-inclusive jets, and $t \bar{t}$ pairs.

\begin{tabular}{|c|c|c|c|c|}
\hline \multirow{2}{*}{$\begin{array}{l}\text { Experiment name } \\
\text { E605'91 }\end{array}$} & \multirow{2}{*}{$\frac{\text { Expt ID }}{201}$} & \multicolumn{2}{|l|}{ Dataset details } & \multirow{2}{*}{$\frac{N_{p t}}{119}$} \\
\hline & & E605 DY & [90] & \\
\hline E866rat'01 & 203 & $\mathrm{E} 866 \mathrm{DY}, \sigma_{p d} /\left(2 \sigma_{p p}\right)$ & [91] & 15 \\
\hline E866pp’03 & 204 & E866 DY, $Q^{3} d^{2} \sigma_{p p} /\left(d Q d x_{F}\right)$ & [92] & 184 \\
\hline CDF1Wasy'96 & 225 & CDF Run-1 $A_{e}\left(\eta^{e}\right)\left(110 \mathrm{pb}^{-1}\right)$ & [93] & 11 \\
\hline CDF2Wasy'05 & 227 & CDF Run-2 $A_{e}\left(\eta^{e}\right)\left(170 \mathrm{pb}^{-1}\right)$ & [94] & 11 \\
\hline D02Masy’08 & 234 & $\mathrm{D} \varnothing$ Run-2 $A_{\mu}\left(\eta^{\mu}\right)\left(0.3 \mathrm{fb}^{-1}\right)$ & [95] & 9 \\
\hline LHCb7WZ'12 & 240 & LHCb $7 \mathrm{TeV} W / Z$ muon forward- $\eta$ Xsec $\left(35 \mathrm{pb}^{-1}\right)$ & [96] & 14 \\
\hline LHCb7Wasy'12 & 241 & LHCb $7 \mathrm{TeV} W A_{\mu}\left(\eta^{\mu}\right)\left(35 \mathrm{pb}^{-1}\right)$ & [96] & 5 \\
\hline ZyD02’08 & 260 & $\mathrm{D} \varnothing$ Run-2 $Z d \sigma / d y_{Z}\left(0.4 \mathrm{fb}^{-1}\right)$ & [97] & 28 \\
\hline ZyCDF2'10 & 261 & CDF Run-2 Z $d \sigma / d y_{Z}\left(2.1 \mathrm{fb}^{-1}\right)$ & [98] & 29 \\
\hline CMS7Masy2’14 & 266 & CMS $7 \mathrm{TeV} A_{u}(\eta)\left(4.7 \mathrm{fb}^{-1}\right)$ & [99] & 11 \\
\hline CMS7Easy'12 & 267 & CMS $7 \mathrm{TeV} A_{e}^{\mu}(\eta)\left(0.840 \mathrm{fb}^{-1}\right)$ & [100] & 11 \\
\hline ATL7WZ'12 & 268 & ATLAS $7 \mathrm{TeV} W / Z \mathrm{Xsec}, A_{\mu}(\eta)\left(35 \mathrm{pb}^{-1}\right)$ & [101] & 41 \\
\hline D02Easy2’15 & 281 & $\mathrm{D} \varnothing \operatorname{Run}-2 A_{e}(\eta)\left(9.7 \mathrm{fb}^{-1}\right)$ & [102] & 13 \\
\hline CDF2jets'09 & 504 & CDF Run- 2 incl. jet $\left(d^{2} \sigma / d p_{T}^{j} d y_{j}\right)\left(1.13 \mathrm{fb}^{-1}\right)$ & [103] & 72 \\
\hline D02jets'08 & 514 & D $\varnothing$ Run-2 incl. jet $\left(d^{2} \sigma / d p_{T}^{j} d y_{j}\right)\left(0.7 \mathrm{fb}^{-1}\right)$ & [104] & 110 \\
\hline ATL7jets'12 & 535 & ATLAS $7 \mathrm{TeV}$ incl. jet $\left(d^{2} \sigma / d p_{T}^{j} d y_{j}\right)\left(35 \mathrm{pb}^{-1}\right)$ & [105] & 90 \\
\hline CMS7jets'13 & 538 & CMS $7 \mathrm{TeV}$ incl. jet $\left(d^{2} \sigma / d p_{T}^{j} d y_{j}\right)\left(5 \mathrm{fb}^{-1}\right)$ & [106] & 133 \\
\hline
\end{tabular}


TABLE IV. Same as Table II, showing experimental datasets for production of vector bosons, single-inclusive jets, and $t \bar{t}$ pairs that were not incorporated in the CT14HERA2 NNLO fit but included in our augmented CTEQ-TEA set.

\begin{tabular}{|c|c|c|c|c|}
\hline Experiment name & Expt ID & Dataset details & & $N_{p t}$ \\
\hline LHCb7ZWrap'15 & 245 & LHCb $7 \mathrm{TeV}$ Z/W muon forward- $\eta$ Xsec $\left(1.0 \mathrm{fb}^{-1}\right)$ & {$[107]$} & 33 \\
\hline LHCb8Zee'15 & 246 & LHCb $8 \mathrm{TeV} \mathrm{Z}$ electron forward $\eta \quad d \sigma / d y_{Z}\left(2.0 \mathrm{fb}^{-1}\right)$ & [108] & 17 \\
\hline ATL7ZpT'14 & 247 & ATLAS $7 \mathrm{TeV} d \sigma / d p_{T}^{Z}\left(4.7 \mathrm{fb}^{-1}\right)$ & [109] & 8 \\
\hline CMS8Wasy'16 & 249 & CMS $8 \mathrm{TeV} \mathrm{W}$ muon, Xsec, $A_{\mu}\left(\eta^{\mu}\right)\left(18.8 \mathrm{fb}^{-1}\right)$ & [110] & 33 \\
\hline LHCb8WZ'16 & 250 & LHCb $8 \mathrm{TeV}$ W/Z muon, Xsec, $A_{\mu}\left(\eta^{\mu}\right)\left(2.0 \mathrm{fb}^{-1}\right)$ & [111] & 42 \\
\hline ATL8DY2D'16 & 252 & ATLAS $8 \mathrm{TeV} \mathrm{Z}\left(d^{2} \sigma / d|y|_{l l} d m_{l l}\right)\left(20.3 \mathrm{fb}^{-1}\right)$ & [112] & 48 \\
\hline ATL8ZpT'16 & 253 & ATLAS $8 \mathrm{TeV}\left(d^{2} \sigma / d p_{T}^{Z} d m_{l l}\right)\left(20.3 \mathrm{fb}^{-1}\right)$ & [113] & 45 \\
\hline CMS7jets'14 & 542 & CMS $7 \mathrm{TeV}$ incl. jet, $\mathrm{R}=0.7,\left(d^{2} \sigma / d p_{T}^{j} d y_{j}\right)\left(5 \mathrm{fb}^{-1}\right)$ & [114] & 158 \\
\hline ATLAS7jets'15 & 544 & ATLAS $7 \mathrm{TeV}$ incl. jet, $\mathrm{R}=0.6,\left(d^{2} \sigma / d p_{T}^{j} d y_{j}\right)\left(4.5 \mathrm{fb}^{-1}\right)$ & [115] & 140 \\
\hline CMS8jets'17 & 545 & CMS $8 \mathrm{TeV}$ incl. jet, $\mathrm{R}=0.7,\left(d^{2} \sigma / d p_{T}^{j} d y_{j}\right)\left(19.7 \mathrm{fb}^{-1}\right)$ & [116] & 185 \\
\hline ATL8ttb-pt'16 & 565 & ATLAS $8 \mathrm{TeV} t \bar{t} d \sigma / d p_{T}^{t}\left(20.3 \mathrm{fb}^{-1}\right)$ & [117] & 8 \\
\hline ATL8ttb-y_ave'16 & 566 & ATLAS $8 \mathrm{TeV} t \bar{t} d \sigma / d y_{\langle t / \bar{t}\rangle}\left(20.3 \mathrm{fb}^{-1}\right)$ & [117] & 5 \\
\hline ATL8ttb-mtt'16 & 567 & ATLAS $8 \mathrm{TeV} t \bar{t} d \sigma / d m_{t \bar{t}}\left(20.3 \mathrm{fb}^{-1}\right)$ & [117] & 7 \\
\hline ATL8ttb-y_ttb'16 & 568 & ATLAS $8 \mathrm{TeV} t \bar{t} d \sigma / d y_{t \bar{t}}\left(20.3 \mathrm{fb}^{-1}\right)$ & [117] & 5 \\
\hline
\end{tabular}

CTEQ-TEA experiments in descending order of their total sensitivity (A, B, C) and point-averaged sensitivity $(1,2,3)$. The conventions that define these A-B-C, and 1-2-3 categories are defined in the caption of Table II. Information on the PDF moments for additional flavor combinations, as well as the numerical values for the total and aggregated sensitivities, can be found on the companion website, Ref. [58].

It is worth noting that there is often a closer correspondence between experiments highlighted in sensitivity maps such as Fig. 3 and those identified in the grid plots for the point-averaged sensitivities $\left\langle\left|S_{f}\right|\right\rangle$, i.e., the upper panels of Figs. 13 and 14. For this reason, in discussing our numerical results as illustrated by the following sensitivity maps, we summarize the highest impact experiments according to complementary considerations of those datasets that enjoy sizable per-datum sensitivities to the PDF moments and those that may not in general possess high-impact points taken in isolation, but are nonetheless predicted to have a large aggregated impact - often by merit of the large number of experimental data points $N_{p t}$ they have.

Our recurring observation in this article has been that, in the CTEQ-TEA global analysis, the experimental data involving nuclear targets affords critical and, in many cases, leading, information on essentially all PDF moments analyzed in Sec. II. This is similarly true of the isovector qPDF examined in Sec. III. Details of the nuclear binding at work in the deuteron, for instance, are relevant for a number of the CTEQ-TEA sets, including BCDMSd'90, NMCrat'97, and E866rat'01. On the other hand, heavier nuclear systems were probed in several other fixed-target experiments, especially those involving $\nu$ DIS; these include CDHSW (both $F_{2}$ and $F_{3}$ sets, measured on $\mathrm{Fe}$ ), the inclusive CCFR and semi-inclusive dimuon data from $\mathrm{NuTeV}$ and CCFR (all also measured on Fe), and the E605 fixed-target $p A$ DrellYan measurements ( $\mathrm{Cu}$ target). In multiple instances-for example, in the impact plots for the strangeness moments $\left\langle x^{1,3}\right\rangle_{s^{+}}$, the $C$-odd combinations $\left\langle x^{2}\right\rangle_{u^{-}, d^{-}}$, and even the gluon total momentum $\langle x\rangle_{g}$-these experiments represent the first, second, or third most influential information by the aggregated or point-averaged sensitivity, or both.

Present phenomenological constraints, particularly at large $x$, are therefore strongly dependent on data for which nuclear corrections are an important consideration. These corrections are imperfectly known, and often dependent on model treatments or an assumption that nuclear correction effects are simply absorbed into extracted PDF uncertainties. An EIC would be well poised to address these issues by performing detailed studies of nuclear medium effects.

\section{IMPLEMENTATION OF LATTICE DATA IN QCD ANALYSES}

In the foregoing sections we have analyzed various empirical constraints upon individual lattice QCD observables which are either presently accessible or expected to be in the foreseeable future. These experimental data were taken either from the CTEQ-TEA high-energy dataset or generated as hypothetical pseudodata recorded at an EIClike $e^{ \pm} p$ DIS collider. The main purpose of this exploration was to identify the experimental processes and measurements that will impose the strongest constraints on the lattice-calculable quantities dependent on the PDFs, and that thus can serve as stringent phenomenological benchmarks to help lattice calculations reach their maturity.

But to this latter point, is it possible to proceed in the direction converse to the one taken by asking: how might the multifaceted results of lattice QCD constrain the $x$ dependence of collinear PDFs fitted in future global analyses? Given the complexity of the multichannel information on the Mellin moments and qPDFs furnished by the lattice, its inclusion in the upcoming PDF fits may produce a plethora of nontrivial constraints on the underlying parametrizations. Rather than attempting to disentangle the 
many potential effects from the implementation of lattice data into a global fit, we will use PDFSense to investigate some general properties, such as the typical momentum fractions constrained by including a specific Mellin moment.

We again consider the moments of the $\mathrm{SU}(2)$ isovector distribution $u-d$, in this case, contrasting the two lowest moments of the $q^{+}$-type distribution, $\langle x\rangle_{u^{+}-d^{+}}$and $\left\langle x^{3}\right\rangle_{u^{+}-d^{+}}$, for which we plot the $\left|S_{f}\right|(x, \mu)$ sensitivity map in the left and right panels of Fig. 15, respectively. We clarify that in Fig. 3 of Sec. II D 1, we examined $\langle x\rangle_{u^{+}-d^{+}}$ and $\left\langle x^{2}\right\rangle_{u^{-}-d^{-}}$, but here we directly examine the effect of incrementing the order of the Mellin moment on a specific flavor/charge combination for the purpose of showcasing the relationship between the order and the associated $x$ dependence of the constraints imposed by data.

In moving from the first moment characterized in Fig. 3 and surrounding text to the third, we find a notable reduction in the point-averaged sensitivity, $\left\langle\left|S_{f}\right|\right\rangle$, of the leading experiment, which remains CMS7Masy2'14 (0.342), but is now immediately succeeded by D02Easy2'15 (0.307). The other leading experiments by per-datum sensitivity also remain E866rat'01 (0.225), CMS7Easy'12 (0.203), and CCFR-F3'97 (0.187), but with significant decreases in their values of $\left\langle\left|S_{f}\right|\right\rangle$ for $\left\langle x^{3}\right\rangle_{u^{+}-d^{+}}$. By inspecting the total sensitivities, an important reordering of the experimental hierarchy becomes evident. In this case, BCDMSp' 89 (39.4), with its large share of measurements at high $x$ and $\mu \sim 10 \mathrm{GeV}$, displaces the combined HERA data, HERAI + II'15 (34.9), in terms of total pull. The important experiments identified by their aggregated sensitivities to $\langle x\rangle_{u^{+}-d^{+}}$ continue to place strong constraints upon $\left\langle x^{3}\right\rangle_{u^{+}-d^{+}}$, with

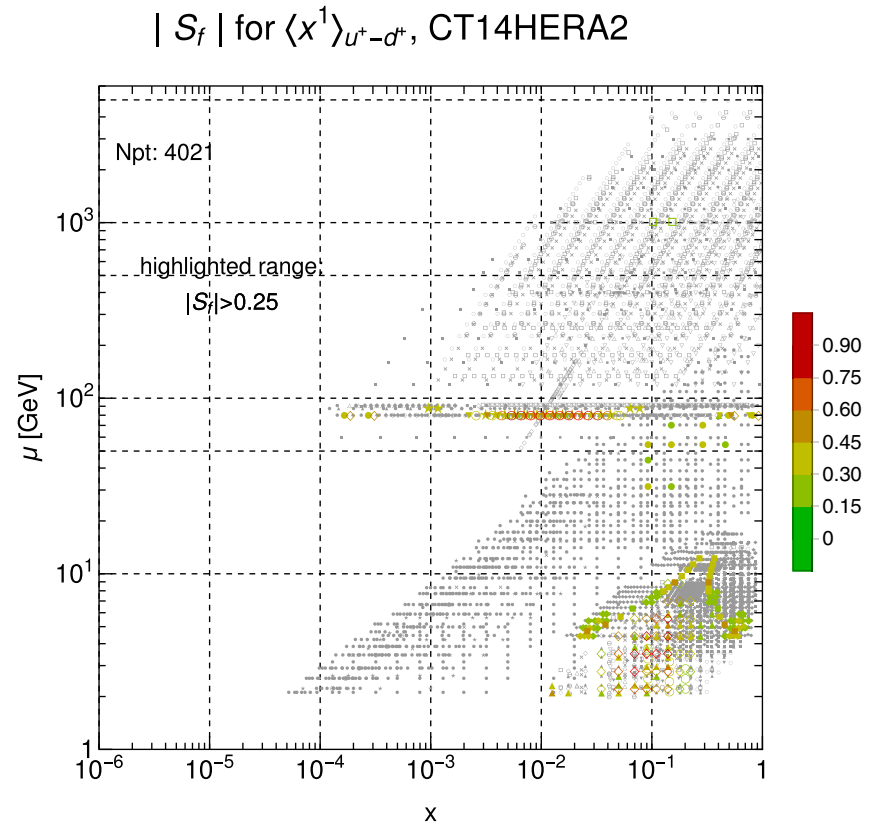

these being E866pp'03 (24.4), CCFR-F3'97 (16.1), and NMCrat' 97 (15.5). For these data, however, there is a salient rightward shift toward higher $x$ in the $(x, \mu)$ space displayed in Fig. 15.

As a straightforward metric to quantify the distribution over $x$ of the sensitivities $\left|S_{f}\right|(x, \mu)$ plotted in Fig. 15, we may evaluate an ensemble average

$$
\bar{x}_{\left|S_{f}\right|}=\frac{\sum_{i} x_{i}\left|S_{f}^{i}\right|}{\sum_{i}\left|S_{f}^{i}\right|},
$$

where the sum $i$ runs over data points in the CTEQ-TEA set. On the basis of this metric and the panels of Fig. 15, a relationship emerges between the order $n$ of the PDF moment $\left\langle x^{n}\right\rangle_{q^{ \pm}}$and the kinematics of the most constraining data in the global analysis, with PDF moments of higher order being constrained more strongly by data recorded at higher $x$. For the first and third isovector moments plotted in Fig. 15, we obtain a systematic increase in $\bar{x}_{\left|S_{f}\right|}$ as the order of the moment is enlarged, finding a shift from $\bar{x}_{\left|S_{f}\right|}=$ 0.193 for $\langle x\rangle_{u^{+}-d^{+}}$to $\bar{x}_{\left|S_{f}\right|}=0.286$ for $\left\langle x^{3}\right\rangle_{u^{+}-d^{+}}$. Similar relationships are observed between $\bar{x}_{\left|S_{f}\right|}$ as given by Eq. (24) and the Mellin moments of other PDF flavors and combinations.

It is possible to further unravel the observed $x$ dependence in $\left|S_{f}\right|(x, \mu)$ by considering the correlation defined in Eq. (15). In Fig. 16, we plot the $x$-dependent correlation between the PDF and its corresponding Mellin moment for two examples - the lowest three lattice-accessible moments of the $d^{+}$distribution (left panel) and the same information for the isovector $u^{+}-d^{+}$. Across both panels, we observe

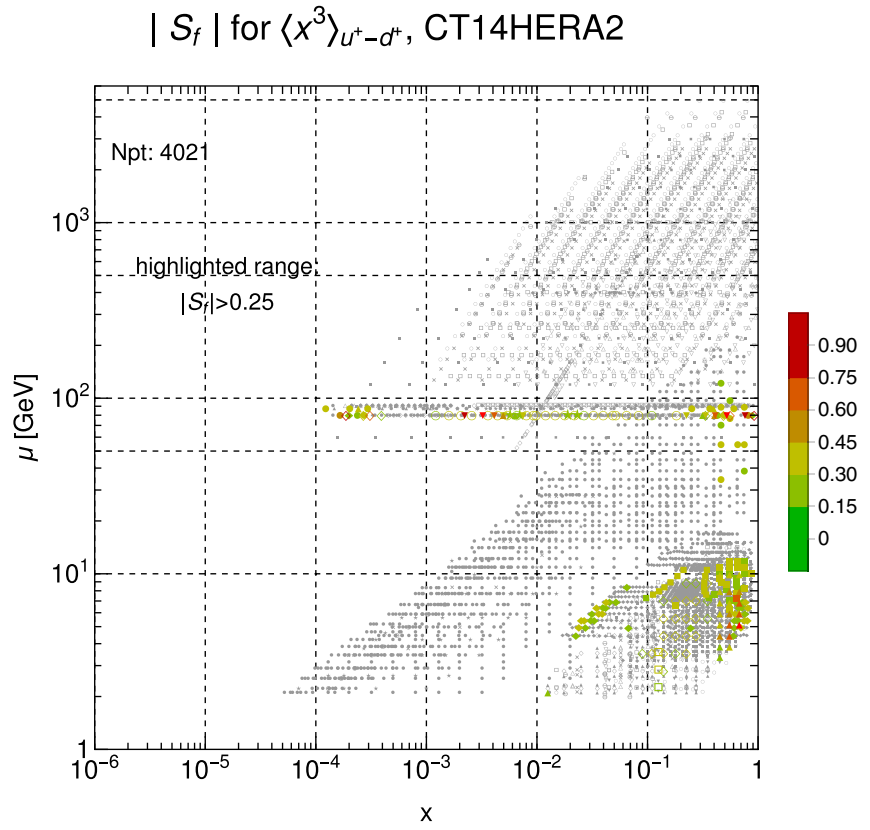

FIG. 15. The two lowest lattice-accessible moments of the isovector PDF combination $u^{+}-d^{+}$. 

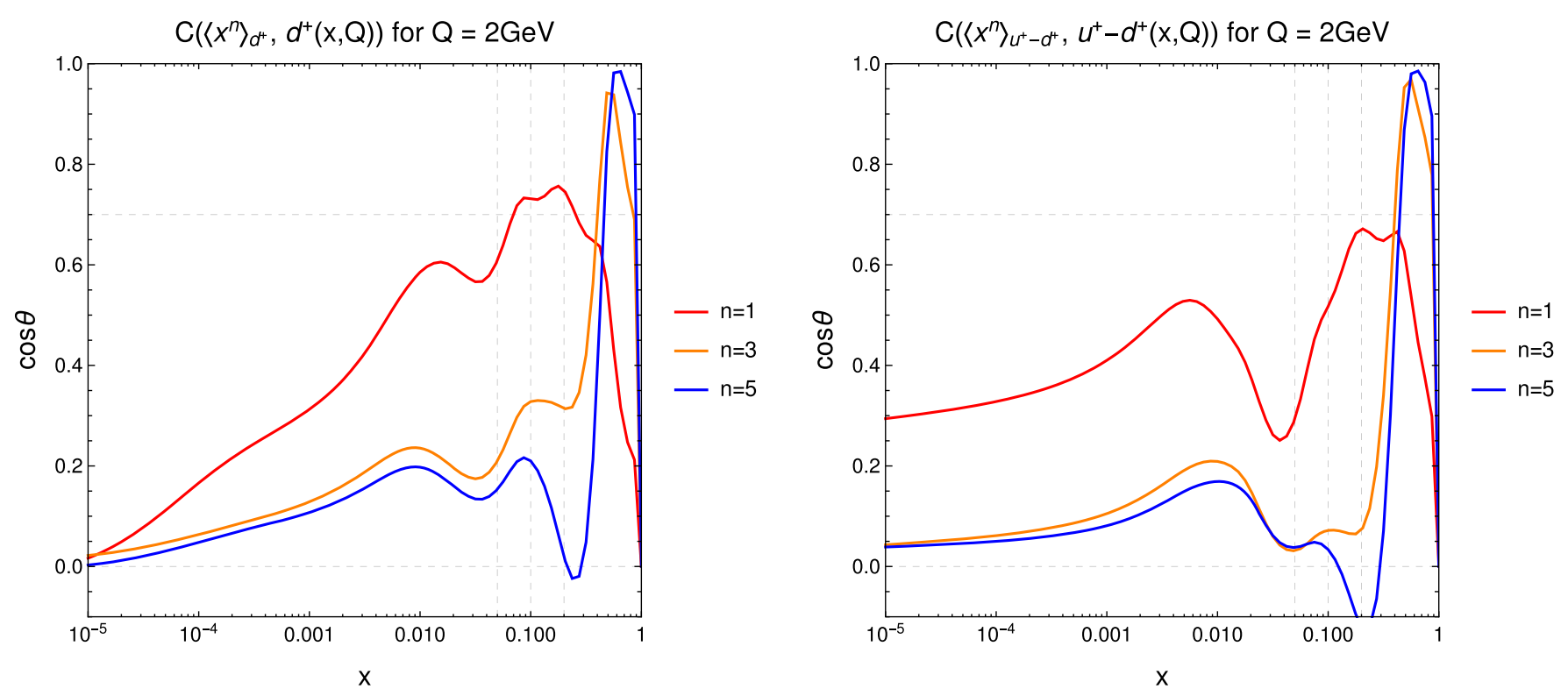

FIG. 16. The correlation between the Mellin moments and their corresponding PDFs for the $d^{+}$(left panel) as well as the isovector $u^{+}-d^{+}$(right panel) moments plotted in Fig. 15. As done elsewhere for the Mellin moment calculations, these are shown for $\mu_{F}=2 \mathrm{GeV}$. For each flavor, we plot the $x$-dependent correlation between the PDF and its integrated moment $\left\langle x^{n}\right\rangle_{q^{+}}$, for $n=1$, 3 , and 5 .

the same qualitative $x$ dependence in the correlation as the order $n$ of the Mellin moment $\left\langle x^{n}\right\rangle_{q^{+}}$is increased. Specifically, while the lowest $n=1$ moment is significantly correlated with its PDF's $x$ dependence over a wide range of $x$, peaking near $x \sim 0.1$, this correlation vanishes rapidly at highest $x$. On the other hand, the PDF correlations with higher moments are rather different, in this case being quite modest, especially for the highest $n=5$ moment, over most of the plotted range before becoming very large, $C_{f} \sim 1$, at $x \gtrsim 0.3$. In fact, this behavior was reflected in Fig. 15, which demonstrated the sensitivity shift in $\left|S_{f}\right|(x, \mu)$ to favor many of the large- $x$ data as the Mellin moment is increased. Taken in conjunction with the correlation results shown in Fig. 16, we may infer that the sensitivity of high- $x$ data to higher moments follows from an underlying reciprocal relation that connects the high- $x$ behavior of PDFs to their higher-order Mellin moments.

The observation that moments $\left\langle x^{n}\right\rangle_{q^{ \pm}}$of successively higher order $(n \geq 1)$ are increasingly sensitive to the PDFs' large- $x$ behavior provides an impetus to seek alternative moment-weighting functions which may be sensitive to low $x$. One possible choice would be successively higher moments of distributions smeared with polynomials in the difference, $(1-x)^{n}$, i.e., $\left\langle(1-x)^{n}\right\rangle_{q^{ \pm}}$. In principle, information on the $\left\langle(1-x)^{n}\right\rangle_{q^{ \pm}}$moments may be of use for constraining PDFs in the region of small $x$, where they must be integrable in the limit $x \rightarrow 0$ to ensure $\left\langle(1-x)^{n}\right\rangle_{q}$ are well defined. In fact, since the polynomial expansion of $(1-x)^{n}$ is a linear combination in $x^{n}$, i.e.,

$$
\begin{aligned}
\left\langle(1-x)^{n}\right\rangle_{q} & =\sum_{k=0}^{n} C_{k}^{n}(-1)^{k}\left\langle x^{k}\right\rangle_{q}, \\
C_{k}^{n} & \equiv \frac{n !}{k !(n-k) !},
\end{aligned}
$$

results for select moments, $\left\langle x^{n}\right\rangle$, provided by the lattice might not only help constrain PDF behavior at successively larger $x$, but might also provide useful supplementary information to assist efforts in the context of QCD global analyses to determine PDF behavior at lower $x$. We clarify this point with a specific example. As argued in Sec. II D 3, the low- $x$ behavior of the isovector difference $u^{+}(x)-$ $d^{+}(x)$ in the integrand of Eq. (22) depends primarily upon the sea-quark flavor asymmetry $\bar{d}-\bar{u}$. By the logic of Eq. (25), a useful quantity with sensitivity to small-x variations in $u^{+}(x)-d^{+}(x)$ might be the weighted moment,

$$
\begin{aligned}
& \left\langle(1-x)^{3}\right\rangle_{u^{+}-d^{+}} \\
& \quad=\langle 1\rangle_{u^{+}-d^{+}}-3\langle x\rangle_{u^{+}-d^{+}}^{\star}+3\left\langle x^{2}\right\rangle_{u^{+}-d^{+}}-\left\langle x^{3}\right\rangle_{u^{+}-d^{+}},
\end{aligned}
$$

in which the two "starred" $\left\langle x^{1,3}\right\rangle$ terms above can in principle be directly informed by lattice calculations. While successively higher moments $\left\langle x^{n}\right\rangle$ are increasingly correlated with PDF behavior at the largest $x \rightarrow 1$, the right panel of Fig. 16 suggests that, through the underlying parametrization, the $\left\langle x^{n}\right\rangle$ moments may nonetheless have significant correlation with the behavior of $u^{+}(x)-d^{+}(x)$ 
for $x \lesssim 10^{-2}$ or smaller-especially for $n=1$. As a result, future PDF analyses seeking to unlock low- $x$ densities may benefit from lattice QCD constraints to select Mellin moments, which, with sufficient precision, might help reduce parametrization dependence and supplement fits to low- $x$ data.

We therefore stress that it will be crucial for future phenomenological analyses to leverage a plurality of lattice results to most strongly constrain their likelihood functions or parameter spaces. The necessity of doing so is apparent from our results on the moments of $\left\langle x^{n}\right\rangle_{u^{+}-d^{+}}$, in which higher moments $(n>1)$ will be useful in coordination with the leading total momentum fractions $\langle x\rangle_{u^{+}-d^{+}}$to maximize constraints over wide reaches of $x$. We also emphasize the fact that the $\left|S_{f}\right|(x, \mu)$ plots imply a synergistic relationship between high-energy and lattice data, with, for example, higher-order moments being especially valuable in informing fitted PDFs' $x$ dependence near $x \approx 1$, where highprecision data can be technically difficult to obtain. Similar logic will place a high premium on lattice results obtained using the quasi-PDF approach.

\section{CONCLUSION}

As the number of observables accessible to lattice QCD continues to grow, the necessity for PDF phenomenologists to grapple with the resulting output will be increasingly unavoidable. The chief message of this article is that this represents an opportunity to form a potentially powerful synergy between phenomenological PDF analyses and lattice QCD. This synergy will be grounded in the ability of PDF phenomenologists to drive improvements in lattice calculations with benchmarks informed by high-energy data, while the lattice provides informative constraints in kinematical regions that are otherwise challenging to constrain empirically. Before the envisioned relationship can be fully realized, however, both communities must establish a common basis for comparing results from lattice QCD and global fits-a challenging undertaking given the complex contemporary landscape of lattice calculations and global fits, which involve a patchwork of theoretical settings, systematic assumptions, and, in the case of QCD analyses, empirical datasets. The PDFSense technology deployed in this article provides a standardized framework in which apples-to-apples evaluations of the pulls of experimental information on lattice-calculable quantities are possible. This fact suggests one avenue for assessing the empirical origins of phenomenological predictions of lattice data and a path forward for improving them.

While some studies have investigated the result of selecting assortments of lattice data for inclusion into a global analysis, in this work we have gone the other direction and examined the constraints data place on quantities which have been computed on the lattice. In the process, we have established several primary findings: (i) We have demonstrated the correspondence between phenomenological predictions for specific physical measurements and the importance of experimental information for benchmarking lattice calculations. Conversely, our impact study in the form of the sensitivity maps for the various lattice observables illustrates those regions of parameter space where improved lattice data can be expected to have a driving impact on PDF studies. For instance, precise lattice data on $\langle x\rangle_{d^{+}}$could improve knowledge of $d^{+}(x)$ in the high- $x$ region, given the sensitivity map of Fig. 4 (right panel) and the correlations plotted in Fig. 16. Similar logic applies to the moments of other flavors.

(ii) In Sec. II, we found that most moments presently accessible to the lattice are mainly constrained by a small collection of high-impact experiments-for many of the light-quark Mellin moments, for instance, a combination of HERA and fixed-target DIS data are especially decisive, as illustrated for the $u^{+}-d^{+}, u^{+}, d^{+}$, and $s^{+}$moments appearing in Figs. 3, 4, and 5, respectively. The overall sensitivities of the CTEQ-TEA experiments to the quantities considered in this analysis are summarized in Figs. 13 and 14, as well as Tables II-IV. The CTEQ-TEA data pulls differ substantially for even vs odd Mellin moments, due to the $C$-oddness vs evenness of the associated quark distributions, seen by comparing the sensitivity maps of Fig. 4 against those of Fig. 6.

(iii) We have also observed systematic tendencies in the sensitivities of high-energy data to Mellin moments, including a robust connection between the order $n$ of the Mellin moments $\left\langle x^{n}\right\rangle_{q^{ \pm}}$of quark distributions and regions of $x$ of the PDFs shown in Fig. 15. This connection is "bidirectional" in the sense that experimental information at higher $x$ are likely to exert stronger pulls on higher-order Mellin moments, while lattice information on the higher-order moments may potentially constrain the high- $x$ behavior of fitted PDFs. We conclude the eventual implementation of lattice data into QCD analyses will benefit from the inclusion of Mellin moments of various orders and parton flavors as well as knowledge gained from qPDFs to constrain PDFs' $x$ dependence as widely as possible.

(iv) We have for the first time studied in Sec. III the driving constraints from high-energy data on calculations of the $P_{z}$-dependent qPDFs. In so doing, we have illustrated the direct link between qPDFs as theoretical quantities and the empirical information upon which calculations of the matched qPDFs from phenomenological distributions depend. One intriguing consequence of this is the possibility of more thoroughly constraining the $P_{z}$ dependence of the phenomenologically matched qPDFs with, e.g., 
DIS measurements concentrated at large $x$ shown in Fig. 10. Reductions to the PDF uncertainties of the high- $x$ qPDFs could help drive theoretical improvements in the LaMET formalism used to extract PDFs from lattice data.

(v) We can assess the potential impact of future experiments such as the high-luminosity lepton-nucleon collider considered in Fig. 12, and we conclude that data from such a machine would be extremely beneficial for advancing the phenomenology of PDF moments and matched qPDFs. The constraints on Mellin moments typically arise from DIS data on fixed nuclear targets, such that nuclear corrections may be an important effect in PDF extractions involving these data. As one example, Sec. II D 2 showed that the gluon Mellin moment $\langle x\rangle_{g}$ receives its largest constraints from DIS data measured on iron nuclei (CCFR, CDHSW), which are known to have a different preference for the large- $x$ gluon than HERA DIS information on the proton. A future lepton-nucleus collider would enlighten such dependence of $\langle x\rangle_{g}$ and other moments on the nuclear environment.

As gains continue to be made on the complementary fronts of lattice theory and QCD analysis in the coming years, the analysis carried out in this work will be of value to guide phenomenologists and lattice practitioners in fully leveraging the synergy between their fields to improve our knowledge of hadron structure. Finally, we note that a comprehensive set of results has been collected at the public URL in Ref. [58]. While this collection includes many of the calculations shown in this manuscript, a range of other sensitivity maps and related computations omitted here for brevity are also shown.

\section{ACKNOWLEDGMENTS}

We are grateful to Yu-Sheng Liu for his assistance regarding the computation of matched quasidistributions from phenomenological fits. We also thank Wally Melnitchouk, Kostas Orginos, Rik Yoshida, and our CTEQ-TEA colleagues for helpful exchanges during the completion of this manuscript. This work was supported by the U.S. Department of Energy under Grant No. DESC0010129. T. J. H. acknowledges support from a JLab EIC Center Fellowship.

\section{APPENDIX: TABULATED SENSITIVITIES}

Similar to Table VI of the PDFSense paper in Ref. [22], the Appendix gives in Tables V, VI, and VII a series of ranking tables of the Mellin moments $\left\langle x^{n}\right\rangle$. Additional tables for other moments and for the isovector quasi-PDF at several values of $x$ and $P_{z}$ appear in the companion website given as Ref. [58].

TABLE V. The aggregated and point-averaged sensitivities to moments of the $u^{ \pm}$-quark distributions of the experiments in the CTEQTEA set, ranking according to the conventions of Ref. [22]. Here and in the subsequent tables, we arrange the CTEQ-TEA experiments in descending order based on their summed sensitivity $\sum\left|S^{E}\right|$ to each of the three moments displayed in the rightmost columns. For each moment, we award especially sensitive experiments a rank A,B,C or 1,2,3 based on their total and point-averaged sensitivities, respectively. These ranks are decided using the criteria: $C \Leftrightarrow\left|S_{f}^{E}\right| \in[4,10], B \Leftrightarrow\left|S_{f}^{E}\right| \in[10,20], A \Leftrightarrow\left|S_{f}^{E}\right| \in[20,50]$, and $A^{*} \Leftrightarrow$ $\left|S_{f}^{E}\right|>50$ according to the total sensitivities for each flavor; and, analogously, $3 \Leftrightarrow\left\langle\left|S_{f}^{E}\right|\right\rangle \in[0.1,0.25], 2 \Leftrightarrow\left\langle\left|S_{f}^{E}\right|\right\rangle \in[0.25,0.5]$, $1 \Leftrightarrow\left\langle\left|S_{f}^{E}\right|\right\rangle \in[0.5,1]$, and $1^{*} \Leftrightarrow\left\langle\left|S_{f}^{E}\right|>1\right.$ according to the point-averaged sensitivities. Experiments with sensitivities falling below the lowest ranks (that is, with $\left|S_{f}^{E}\right|<4$ or $\left\langle\left|S_{f}^{E}\right|\right\rangle<0.1$ ) are not awarded a rank for that moment.

\begin{tabular}{|c|c|c|c|c|c|c|c|c|c|c|}
\hline No. & Expt ID & $N_{p t}$ & $\sum\left|S^{E}\right|$ & $\left\langle\sum\left|S^{E}\right|\right\rangle$ & $\left|S_{\left\langle x^{1}\right\rangle_{u^{+}}}\right|$ & $\left\langle\left|S_{\left\langle x^{1}\right\rangle_{u^{+}}}\right|\right\rangle$ & $\left|S_{\left\langle x^{2}\right\rangle_{u^{-}}}\right|$ & $\left\langle\left|S_{\left\langle x^{2}\right\rangle_{u}}\right|\right\rangle$ & $\left|S_{\left\langle x^{3}\right\rangle_{u^{+}}}\right|$ & $\left\langle\left|S_{\left\langle x^{3}\right\rangle_{u^{+}}}\right|\right\rangle$ \\
\hline 1 & BCDMSp'89 & 337 & 125.0 & 0.123 & A & 3 & A & 3 & A & 3 \\
\hline 2 & HERAI + II' 15 & 1120.0 & 122.0 & 0.0363 & A & & A & & A & \\
\hline 3 & CCFR-F3’97 & 86 & 95.8 & 0.371 & A & 2 & A & 1 & A & 2 \\
\hline 4 & E866pp'03 & 184 & 69.3 & 0.125 & B & & A & 3 & A & 3 \\
\hline 5 & BCDMSd'90 & 250 & 55.3 & 0.0737 & A & & B & & B & \\
\hline 6 & CDHSW-F3'91 & 96 & 52.7 & 0.183 & B & 3 & A & 3 & B & 3 \\
\hline 7 & CDHSW-F2'91 & 85 & 35.7 & 0.14 & B & 3 & $\mathrm{C}$ & 3 & B & 3 \\
\hline 8 & NMCrat'97 & 123 & 34.5 & 0.0934 & $\mathrm{C}$ & & B & 3 & B & 3 \\
\hline 9 & CCFR-F2’01 & 69 & 31.5 & 0.152 & $\mathrm{C}$ & 3 & $\mathrm{C}$ & 3 & B & 3 \\
\hline 10 & CMS7jets'13 & 133 & 27.5 & 0.0689 & $\mathrm{C}$ & & $\mathrm{C}$ & & B & \\
\hline 11 & CMS8jets'17 & 185 & 25.3 & 0.0456 & $\mathrm{C}$ & & $\mathrm{C}$ & & B & \\
\hline 12 & E605’91 & 119 & 24.0 & 0.0671 & & & B & 3 & & \\
\hline 13 & CMS7jets'14 & 158 & 19.5 & 0.0411 & $\mathrm{C}$ & & $\mathrm{C}$ & & $\mathrm{C}$ & \\
\hline 14 & ATLAS7jets'15 & 140 & 16.2 & 0.0387 & & & $\mathrm{C}$ & & $\mathrm{C}$ & \\
\hline 15 & NuTeV-nu’06 & 38 & 12.9 & 0.113 & $\mathrm{C}$ & 3 & & & & \\
\hline 16 & LHCb8WZ'16 & 42 & 12.5 & 0.0989 & & & & & $\mathrm{C}$ & 3 \\
\hline
\end{tabular}

(Table continued) 
TABLE V. (Continued)

\begin{tabular}{|c|c|c|c|c|c|c|c|c|c|c|}
\hline No. & Expt ID & $N_{p t}$ & $\sum\left|S^{E}\right|$ & $\left\langle\sum\left|S^{E}\right|\right\rangle$ & $\left|S_{\left\langle x^{1}\right\rangle_{u^{+}}}\right|$ & $\left\langle\left|S_{\left\langle x^{1}\right\rangle_{u^{+}}}\right|\right\rangle$ & $\left|S_{\left\langle x^{2}\right\rangle_{u^{-}}}\right|$ & $\left\langle\left|S_{\left\langle x^{2}\right\rangle_{u^{-}}}\right|\right\rangle$ & $\left|S_{\left\langle x^{3}\right\rangle_{u^{+}}}\right|$ & $\left\langle\left|S_{\left\langle x^{3}\right\rangle_{u^{+}}}\right|\right\rangle$ \\
\hline 17 & CCFR SI nub'01 & 38 & 11.9 & 0.104 & $\mathrm{C}$ & 3 & & & & \\
\hline 18 & NuTeV-nub’06 & 33 & 11.8 & 0.119 & $\mathrm{C}$ & 3 & & & & 3 \\
\hline 19 & CMS7Masy2’14 & 11 & 11.5 & 0.349 & & 2 & $\mathrm{C}$ & 2 & $\mathrm{C}$ & 2 \\
\hline 20 & LHCb7ZWrap'15 & 33 & 11.4 & 0.115 & & & & 3 & $\mathrm{C}$ & 3 \\
\hline 21 & ATL7jets'12 & 90 & 11.1 & 0.0412 & & & & & $\mathrm{C}$ & \\
\hline 22 & ATL8DY2D'16 & 48 & 10.2 & 0.0706 & $\mathrm{C}$ & & & & & \\
\hline 23 & D02jets'08 & 110 & 10.2 & 0.0308 & & & & & $\mathrm{C}$ & \\
\hline 24 & E866rat'01 & 15 & 10.0 & 0.223 & $\mathrm{C}$ & 2 & & 3 & & 3 \\
\hline 25 & CCFR SI nu'01 & 40 & 9.71 & 0.0809 & $\mathrm{C}$ & 3 & & & & \\
\hline 26 & CDF2jets’09 & 72 & 8.51 & 0.0394 & & & & & & \\
\hline 27 & CMS8Wasy'16 & 33 & 7.18 & 0.0726 & & & & & & \\
\hline 28 & ATL7WZ'12 & 41 & 6.97 & 0.0567 & & & & & & \\
\hline 29 & CMS7Easy'12 & 11 & 6.81 & 0.206 & & 3 & & 3 & & 3 \\
\hline 30 & D02Easy2’15 & 13 & 6.46 & 0.166 & & & & 3 & & 2 \\
\hline 31 & ATL8ZpT'16 & 45 & 5.21 & 0.0386 & & & & & & \\
\hline 32 & ZyCDF2’10 & 29 & 4.88 & 0.0561 & & & & & & \\
\hline 33 & ZyD02’08 & 28 & 4.61 & 0.0549 & & & & & & \\
\hline 34 & D02Masy’08 & 9 & 4.15 & 0.154 & & 2 & & & & 3 \\
\hline 35 & LHCb8Zee'15 & 17 & 3.32 & 0.065 & & & & & & \\
\hline 36 & HERAc'13 & 47 & 3.15 & 0.0224 & & & & & & \\
\hline 37 & CDF1Wasy'96 & 11 & 2.75 & 0.0835 & & & & & & 3 \\
\hline 38 & LHCb7WZ'12 & 14 & 2.34 & 0.0557 & & & & & & \\
\hline 39 & ATL8ttb-mtt' 16 & 7 & 1.2 & 0.0573 & & & & & & \\
\hline 40 & ATL8ttb-pt'16 & 8 & 1.17 & 0.0489 & & & & & & \\
\hline 41 & LHCb7Wasy'12 & 5 & 1.17 & 0.0781 & & & & & & 3 \\
\hline 42 & ATL7ZpT'14 & 8 & 1.04 & 0.0435 & & 3 & & & & \\
\hline 43 & CDF2Wasy’05 & 11 & 1.02 & 0.0311 & & & & & & \\
\hline 44 & ATL8ttb-y_ave'16 & 5 & 0.548 & 0.0365 & & & & & & \\
\hline 45 & HERA-FL'11 & 9 & 0.508 & 0.0188 & & & & & & \\
\hline 46 & ATL8ttb-y_ttb'16 & 5 & 0.495 & 0.033 & & & & & & \\
\hline 47 & HERAb'06 & 10 & 0.328 & 0.0109 & & & & & & \\
\hline
\end{tabular}

TABLE VI. The sensitivities to moments of the $d^{ \pm}$-quark distributions of the CTEQ-TEA experiments in the CTEQ-TEA set.

\begin{tabular}{|c|c|c|c|c|c|c|c|c|c|c|}
\hline No. & Expt ID & $N_{p t}$ & $\sum\left|S^{E}\right|$ & $\left\langle\sum\left|S^{E}\right|\right\rangle$ & $\left|S_{\left\langle x^{1}\right\rangle_{d^{+}}}\right|$ & $\left\langle\left|S_{\left\langle x^{1}\right\rangle_{d^{+}}}\right|\right\rangle$ & $\left|S_{\left\langle x^{2}\right\rangle_{d^{-}}}\right|$ & $\left\langle\left|S_{\left\langle x^{2}\right\rangle_{d^{-}}}\right|\right\rangle$ & $\left|S_{\left\langle x^{3}\right\rangle_{d^{+}}}\right|$ & $\left\langle\left|S_{\left\langle x^{3}\right\rangle_{d^{+}}}\right|\right\rangle$ \\
\hline 1 & HERAI + II'15 & 1120.0 & 116.0 & 0.0346 & $A^{*}$ & & A & & A & \\
\hline 2 & CCFR-F3'97 & 86 & 70.3 & 0.272 & $\mathrm{~B}$ & 3 & A & 2 & $\mathrm{~B}$ & 3 \\
\hline 3 & BCDMSd'90 & 250 & 58.8 & 0.0784 & A & 3 & $\mathrm{~B}$ & & $\mathrm{~B}$ & \\
\hline 4 & NMCrat'97 & 123 & 55.6 & 0.151 & A & 3 & $\mathrm{~B}$ & 3 & $\mathrm{~B}$ & 3 \\
\hline 5 & BCDMSp'89 & 337 & 53.8 & 0.0532 & $\mathrm{~B}$ & & $\mathrm{~B}$ & & A & \\
\hline 6 & E866pp’03 & 184 & 44.2 & 0.08 & $\mathrm{~B}$ & & $\mathrm{~B}$ & & $\mathrm{~B}$ & \\
\hline 7 & CDHSW-F2'91 & 85 & 43.5 & 0.171 & $\mathrm{~B}$ & 3 & $\mathrm{~B}$ & 3 & $\mathrm{~B}$ & 3 \\
\hline 8 & E605'91 & 119 & 37.3 & 0.105 & $\mathrm{C}$ & & A & 3 & $\mathrm{C}$ & \\
\hline 9 & CDHSW-F3'91 & 96 & 36.2 & 0.126 & $\mathrm{C}$ & & $\mathrm{B}$ & 3 & $\mathrm{C}$ & \\
\hline 10 & CCFR-F2'01 & 69 & 27.4 & 0.133 & $\mathrm{~B}$ & 3 & $\mathrm{C}$ & 3 & $\mathrm{C}$ & 3 \\
\hline 11 & LHCb8WZ'16 & 42 & 16.4 & 0.13 & & & $\mathrm{C}$ & 3 & $\mathrm{C}$ & 3 \\
\hline 12 & CMS7jets'13 & 133 & 16.2 & 0.0405 & $\mathrm{C}$ & & $\mathrm{C}$ & & $\mathrm{C}$ & \\
\hline 13 & CMS8jets'17 & 185 & 15.2 & 0.0273 & $\mathrm{C}$ & & & & $\mathrm{C}$ & \\
\hline 14 & LHCb7ZWrap'15 & 33 & 14.0 & 0.141 & & & $\mathrm{C}$ & 3 & $\mathrm{C}$ & 3 \\
\hline 15 & CMS7jets'14 & 158 & 13.4 & 0.0283 & $\mathrm{C}$ & & & & $\mathrm{C}$ & \\
\hline 16 & D02Easy2'15 & 13 & 12.0 & 0.309 & & 3 & $\mathrm{C}$ & 1 & & 2 \\
\hline 17 & NuTeV-nu’06 & 38 & 11.7 & 0.103 & $\mathrm{C}$ & 3 & & & & \\
\hline 18 & CMS7Masy2’14 & 11 & 10.2 & 0.308 & $\mathrm{C}$ & 2 & & 2 & & 3 \\
\hline
\end{tabular}


TABLE VI. (Continued)

\begin{tabular}{|c|c|c|c|c|c|c|c|c|c|c|}
\hline No. & Expt ID & $N_{p t}$ & $\sum\left|S^{E}\right|$ & $\left\langle\sum\left|S^{E}\right|\right\rangle$ & $\left|S_{\left\langle x^{1}\right\rangle_{d^{+}}}\right|$ & $\left\langle\left|S_{\left\langle x^{1}\right\rangle_{d^{+}}}\right|\right\rangle$ & $\left|S_{\left\langle x^{2}\right\rangle_{d^{-}}}\right|$ & $\left\langle\left|S_{\left\langle x^{2}\right\rangle_{d^{-}}}\right|\right\rangle$ & $\left|S_{\left\langle x^{3}\right\rangle_{d^{+}}}\right|$ & $\left\langle\left|S_{\left\langle x^{3}\right\rangle_{d^{+}}}\right|\right\rangle$ \\
\hline 19 & ATLAS7jets'15 & 140 & 9.11 & 0.0217 & & & & & $\mathrm{C}$ & \\
\hline 20 & ATL8DY2D'16 & 48 & 8.82 & 0.0612 & & & & & & \\
\hline 21 & CCFR SI nu'01 & 40 & 8.61 & 0.0718 & $\mathrm{C}$ & 3 & & & & \\
\hline 22 & E866rat'01 & 15 & 8.48 & 0.188 & & 3 & & 3 & & 3 \\
\hline 23 & ATL7WZ'12 & 41 & 8.38 & 0.0681 & & & & & & \\
\hline 24 & CCFR SI nub'01 & 38 & 7.62 & 0.0668 & $\mathrm{C}$ & 3 & & & & \\
\hline 25 & CMS8Wasy'16 & 33 & 6.99 & 0.0706 & & & & & & \\
\hline 26 & NuTeV-nub'06 & 33 & 6.68 & 0.0674 & $\mathrm{C}$ & 3 & & & & \\
\hline 27 & ATL7jets'12 & 90 & 6.61 & 0.0245 & & & & & & \\
\hline 28 & D02jets'08 & 110 & 6.14 & 0.0186 & & & & & & \\
\hline 29 & CMS7Easy'12 & 11 & 5.72 & 0.173 & & 3 & & 3 & & 3 \\
\hline 30 & CDF1Wasy'96 & 11 & 5.12 & 0.155 & & 3 & & 2 & & \\
\hline 31 & ATL8ZpT'16 & 45 & 4.99 & 0.037 & & & & & & \\
\hline 32 & D02Masy'08 & 9 & 4.35 & 0.161 & & 3 & & 2 & & \\
\hline 33 & ZyD02’08 & 28 & 3.8 & 0.0452 & & & & & & \\
\hline 34 & LHCb7WZ'12 & 14 & 3.77 & 0.0898 & & & & 3 & & \\
\hline 35 & CDF2jets'09 & 72 & 3.58 & 0.0166 & & & & & & \\
\hline 36 & ZyCDF2’10 & 29 & 3.06 & 0.0352 & & & & & & \\
\hline 37 & LHCb7Wasy'12 & 5 & 2.78 & 0.185 & & & & 2 & & 3 \\
\hline 38 & ATL7ZpT’14 & 8 & 2.34 & 0.0976 & & 3 & & & & \\
\hline 39 & HERAc'13 & 47 & 1.97 & 0.014 & & & & & & \\
\hline 40 & CDF2Wasy'05 & 11 & 1.86 & 0.0565 & & & & & & \\
\hline 41 & LHCb8Zee'15 & 17 & 1.63 & 0.032 & & & & & & \\
\hline 42 & ATL8ttb-pt'16 & 8 & 1.47 & 0.0614 & & 3 & & & & \\
\hline 43 & ATL8ttb-mtt'16 & 7 & 1.37 & 0.0654 & & & & & & \\
\hline 44 & ATL8ttb-y_ave'16 & 5 & 0.776 & 0.0517 & & & & & & \\
\hline 45 & ATL8ttb-y_ttb'16 & 5 & 0.461 & 0.0307 & & & & & & \\
\hline 46 & HERA-FL'11 & 9 & 0.407 & 0.0151 & & & & & & \\
\hline 47 & HERAb'06 & 10 & 0.252 & 0.0084 & & & & & & \\
\hline
\end{tabular}

TABLE VII. The sensitivities to moments of the $u^{ \pm}-d^{ \pm}$isovector quark distributions of the CTEQ-TEA experiments in the CTEQTEA set.

\begin{tabular}{|c|c|c|c|c|c|c|c|c|c|c|}
\hline No. & Expt ID & $N_{p t}$ & $\sum\left|S^{E}\right|$ & $\left\langle\sum\left|S^{E}\right|\right\rangle$ & $\left|S_{\left\langle x^{1}\right\rangle_{u^{+}-d^{+}}}\right|$ & $\left\langle\left|S_{\left\langle x^{1}\right\rangle_{u^{+}-d^{+}}}\right|\right\rangle$ & $\left|S_{\left\langle x^{2}\right\rangle_{u^{-}-d^{-}}}\right|$ & $\left\langle\left|S_{\left\langle x^{2}\right\rangle_{u^{-}-d^{-}}}\right|\right\rangle$ & $\left|S_{\left\langle x^{3}\right\rangle_{u^{+}-d^{+}}}\right|$ & $\left\langle\left|S_{\left\langle x^{3}\right\rangle_{u^{+}-d^{+}}}\right|\right\rangle$ \\
\hline 1 & HERAI + II'15 & 1120.0 & 109.0 & 0.0325 & A & & A & & A & \\
\hline 2 & BCDMSp'89 & 337 & 91.9 & 0.0909 & B & & A & & A & 3 \\
\hline 3 & E866pp’03 & 184 & 67.3 & 0.122 & A & 3 & $\mathrm{~A}$ & 3 & A & 3 \\
\hline 4 & CCFR-F3'97 & 86 & 61.9 & 0.24 & A & 2 & B & 3 & $\mathrm{~B}$ & 3 \\
\hline 5 & NMCrat'97 & 123 & 60.0 & 0.163 & A & 3 & B & 3 & B & 3 \\
\hline 6 & CDHSW-F3'91 & 96 & 27.4 & 0.0953 & $\mathrm{~B}$ & 3 & $\mathrm{C}$ & & $\mathrm{C}$ & \\
\hline 7 & BCDMSd'90 & 250 & 25.5 & 0.034 & $\mathrm{C}$ & & $\mathrm{C}$ & & $\mathrm{C}$ & \\
\hline 8 & LHCb8WZ'16 & 42 & 15.3 & 0.122 & $\mathrm{C}$ & & $\mathrm{C}$ & 3 & $\mathrm{C}$ & 3 \\
\hline 9 & CMS7Masy2’14 & 11 & 15.3 & 0.463 & $\mathrm{C}$ & 1 & $\mathrm{C}$ & 2 & & 2 \\
\hline 10 & CCFR-F2'01 & 69 & 15.2 & 0.0733 & $\mathrm{C}$ & & $\mathrm{C}$ & & $\mathrm{C}$ & \\
\hline 11 & E605'91 & 119 & 15.1 & 0.0423 & $\mathrm{C}$ & & & & $\mathrm{C}$ & \\
\hline 12 & CMS8jets'17 & 185 & 14.1 & 0.0254 & & & $\mathrm{C}$ & & $\mathrm{C}$ & \\
\hline 13 & LHCb7ZWrap'15 & 33 & 12.9 & 0.13 & $\mathrm{C}$ & 3 & $\mathrm{C}$ & 3 & & 3 \\
\hline 14 & D02Easy2’15 & 13 & 11.6 & 0.298 & & 3 & $\mathrm{C}$ & 2 & & 2 \\
\hline 15 & CDHSW-F2'91 & 85 & 11.4 & 0.0449 & $\mathrm{C}$ & & & & & \\
\hline 16 & E866rat'01 & 15 & 11.3 & 0.251 & $\mathrm{C}$ & 2 & & 3 & & 3 \\
\hline 17 & CMS7jets'13 & 133 & 11.2 & 0.0282 & & & $\mathrm{C}$ & & & \\
\hline 18 & ATL8DY2D’16 & 48 & 11.1 & 0.0772 & $\mathrm{C}$ & & & & & \\
\hline 19 & CMS7Easy'12 & 11 & 8.98 & 0.272 & & 2 & & 2 & & 3 \\
\hline
\end{tabular}


TABLE VII. (Continued)

\begin{tabular}{|c|c|c|c|c|c|c|c|c|c|c|}
\hline No. & Expt ID & $N_{p t}$ & $\sum\left|S^{E}\right|$ & $\left\langle\sum\left|S^{E}\right|\right\rangle$ & $\left|S_{\left\langle x^{1}\right\rangle_{u^{+}-d^{+}}}\right|$ & $\left\langle\left|S_{\left\langle x^{1}\right\rangle_{u^{+}-d^{+}}}\right|\right\rangle$ & $\left|S_{\left\langle x^{2}\right\rangle_{u^{-}-d^{-}}}\right|$ & $\left\langle\left|S_{\left\langle x^{2}\right\rangle_{u^{-}-d^{-}}}\right|\right\rangle$ & $\left|S_{\left\langle x^{3}\right\rangle_{u^{+}-d^{+}}}\right|$ & $\left\langle\left|S_{\left\langle x^{3}\right\rangle_{u^{+}-d^{+}}}\right|\right\rangle$ \\
\hline 20 & CMS8Wasy'16 & 33 & 8.77 & 0.0886 & & 3 & & & & \\
\hline 21 & ATL7WZ'12 & 41 & 8.64 & 0.0703 & & & & & & \\
\hline 22 & CMS7jets'14 & 158 & 8.54 & 0.018 & & & & & & \\
\hline 23 & ATLAS7jets'15 & 140 & 8.07 & 0.0192 & & & & & & \\
\hline 24 & CDF2jets'09 & 72 & 5.22 & 0.0241 & & & & & & \\
\hline 25 & D02jets'08 & 110 & 5.0 & 0.0151 & & & & & & \\
\hline 26 & NuTeV-nu'06 & 38 & 4.99 & 0.0437 & & & & & & \\
\hline 27 & ATL7jets'12 & 90 & 4.84 & 0.0179 & & & & & & \\
\hline 28 & CDF1Wasy'96 & 11 & 4.83 & 0.146 & & 3 & & 3 & & 3 \\
\hline 29 & NuTeV-nub'06 & 33 & 4.28 & 0.0432 & & & & & & \\
\hline 30 & D02Masy’08 & 9 & 4.2 & 0.155 & & 3 & & 3 & & 3 \\
\hline 31 & CCFR SI nub'01 & 38 & 3.71 & 0.0325 & & & & & & \\
\hline 32 & CCFR SI nu'01 & 40 & 3.61 & 0.0301 & & & & & & \\
\hline 33 & ZyCDF2’10 & 29 & 3.58 & 0.0411 & & & & & & \\
\hline 34 & ATL8ZpT'16 & 45 & 3.29 & 0.0243 & & & & & & \\
\hline 35 & ZyD02’08 & 28 & 3.11 & 0.0371 & & & & & & \\
\hline 36 & LHCb7WZ'12 & 14 & 2.88 & 0.0685 & & & & & & \\
\hline 37 & LHCb7Wasy'12 & 5 & 2.47 & 0.165 & & & & 2 & & 3 \\
\hline 38 & HERAc'13 & 47 & 1.79 & 0.0127 & & & & & & \\
\hline 39 & LHCb8Zee'15 & 17 & 1.74 & 0.034 & & & & & & \\
\hline 40 & CDF2Wasy'05 & 11 & 1.73 & 0.0524 & & & & & & \\
\hline 41 & ATL7ZpT'14 & 8 & 1.2 & 0.05 & & & & & & \\
\hline 42 & ATL8ttb-pt'16 & 8 & 0.951 & 0.0396 & & & & & & \\
\hline 43 & ATL8ttb-mtt'16 & 7 & 0.517 & 0.0246 & & & & & & \\
\hline 44 & ATL8ttb-y_ave'16 & 5 & 0.236 & 0.0157 & & & & & & \\
\hline 45 & ATL8ttb-y_ttb'16 & 5 & 0.164 & 0.0109 & & & & & & \\
\hline 46 & HERAb'06 & 10 & 0.162 & 0.0054 & & & & & & \\
\hline 47 & HERA-FL'11 & 9 & 0.15 & 0.00554 & & & & & & \\
\hline
\end{tabular}

[1] A. Accardi, L. T. Brady, W. Melnitchouk, J. F. Owens, and N. Sato, Phys. Rev. D 93, 114017 (2016).

[2] L. A. Harland-Lang, A. D. Martin, P. Motylinski, and R. S. Thorne, Eur. Phys. J. C 75, 204 (2015).

[3] R. D. Ball et al. (NNPDF Collaboration), Eur. Phys. J. C 77, 663 (2017).

[4] S. Dulat, T.-J. Hou, J. Gao, M. Guzzi, J. Huston, P. Nadolsky, J. Pumplin, C. Schmidt, D. Stump, and C.-P. Yuan, Phys. Rev. D 93, 033006 (2016).

[5] T.-J. Hou, S. Dulat, J. Gao, M. Guzzi, J. Huston, P. Nadolsky, J. Pumplin, C. Schmidt, D. Stump, and C.-P. Yuan, Phys. Rev. D 95, 034003 (2017).

[6] J. Speth and A. W. Thomas, Adv. Nucl. Phys. 24, 83 (1997).

[7] S. Kumano, Phys. Rep. 303, 183 (1998).

[8] C. Bourrely, J. Soffer, and F. Buccella, Eur. Phys. J. C 23, 487 (2002).

[9] R. J. Holt and C. D. Roberts, Rev. Mod. Phys. 82, 2991 (2010).

[10] H. Avakian, A. V. Efremov, P. Schweitzer, and F. Yuan, Phys. Rev. D 81, 074035 (2010).
[11] K. D. Bednar, I. C. Cloët, and P. C. Tandy, Phys. Lett. B 782, 675 (2018).

[12] T. J. Hobbs, M. Alberg, and G. A. Miller, Phys. Rev. C 91, 035205 (2015).

[13] W. Melnitchouk and M. Malheiro, Phys. Lett. B 451, 224 (1999).

[14] T. J. Hobbs, J. T. Londergan, and W. Melnitchouk, Phys. Rev. D 89, 074008 (2014).

[15] T. J. Hobbs, M. Alberg, and G. A. Miller, Phys. Rev. D 96, 074023 (2017).

[16] J. Gao, L. Harland-Lang, and J. Rojo, Phys. Rep. 742, 1 (2018).

[17] K. Kovařík, P. M. Nadolsky, and D. E. Soper, arXiv: 1905.06957.

[18] H.-W. Lin et al., Prog. Part. Nucl. Phys. 100, 107 (2018).

[19] W. Detmold, W. Melnitchouk, and A. W. Thomas, Mod. Phys. Lett. A 18, 2681 (2003).

[20] X. Ji, Phys. Rev. Lett. 110, 262002 (2013).

[21] A. V. Radyushkin, Phys. Rev. D 96, 034025 (2017).

[22] B.-T. Wang, T. J. Hobbs, S. Doyle, J. Gao, T.-J. Hou, P. M. Nadolsky, and F. I. Olness, Phys. Rev. D 98, 094030 (2018). 
[23] W. Zimmermann, Ann. Phys. (N.Y.) 77, 570 (1973); Lect. Notes Phys. 558, 278 (2000).

[24] K. G. Wilson, Phys. Rev. 179, 1499 (1969).

[25] N. H. Christ, B. Hasslacher, and A. H. Mueller, Phys. Rev. D 6, 3543 (1972).

[26] D. J. Gross and F. Wilczek, Phys. Rev. D 8, 3633 (1973).

[27] D. J. Gross and F. Wilczek, Phys. Rev. D 9, 980 (1974).

[28] H. Georgi and H. D. Politzer, Phys. Rev. D 9, 416 (1974).

[29] H. Georgi and H. D. Politzer, Phys. Rev. D 14, 1829 (1976).

[30] J. Blümlein and H. Bottcher, Phys. Lett. B 662, 336 (2008).

[31] M. Gockeler, R. Horsley, E.-M. Ilgenfritz, H. Perlt, P. E. L. Rakow, G. Schierholz, and A. Schiller, Phys. Rev. D 53, 2317 (1996).

[32] W. Detmold, W. Melnitchouk, and A. W. Thomas, Eur. Phys. J. direct C 3, 1 (2001).

[33] W. Detmold, W. Melnitchouk, and A. W. Thomas, Phys. Rev. D 68, 034025 (2003).

[34] S. Aoki et al., Eur. Phys. J. C 77, 112 (2017).

[35] M. Gockeler, R. Horsley, E.-M. Ilgenfritz, H. Perlt, P. E. L. Rakow, G. Schierholz, and A. Schiller, Phys. Rev. D 54, 5705 (1996)

[36] C. Alexandrou, M. Constantinou, K. Hadjiyiannakou, K. Jansen, C. Kallidonis, G. Koutsou, A. V. Avilés-Casco, and C. Wiese, Phys. Rev. Lett. 119, 142002 (2017).

[37] G. S. Bali, S. Collins, B. Gläßle, M. Göckeler, J. Najjar, R. H. Rödl, A. Schäfer, R. W. Schiel, A. Sternbeck, and W. Söldner, Phys. Rev. D 90, 074510 (2014).

[38] J. R. Green, M. Engelhardt, S. Krieg, J. W. Negele, A. V. Pochinsky, and S. N. Syritsyn, Phys. Lett. B 734, 290 (2014).

[39] M. Gockeler, R. Horsley, D. Pleiter, P. E. L. Rakow, and G. Schierholz (QCDSF Collaboration), Phys. Rev. D 71, 114511 (2005).

[40] D. Dolgov et al. (LHPC and TXL Collaborations), Phys. Rev. D 66, 034506 (2002).

[41] M. Deka, T. Streuer, T. Doi, S. J. Dong, T. Draper, K. F. Liu, N. Mathur, and A. W. Thomas, Phys. Rev. D 79, 094502 (2009).

[42] J. Liang, M. Sun, Y.-B. Yang, T. Draper, and K.-F. Liu, arXiv:1901.07526.

[43] C. P. Yuan et al., Proc. Sci. DIS2019 (2019) 001.

[44] J. Pumplin, D. R. Stump, J. Huston, H. L. Lai, P. M. Nadolsky, and W. K. Tung, J. High Energy Phys. 07 (2002) 012.

[45] J. Pumplin, D. Stump, R. Brock, D. Casey, J. Huston, J. Kalk, H. L. Lai, and W. K. Tung, Phys. Rev. D 65, 014013 (2001).

[46] D. Stump, J. Pumplin, R. Brock, D. Casey, J. Huston, J. Kalk, H. L. Lai, and W. K. Tung, Phys. Rev. D 65, 014012 (2001).

[47] J. Pumplin, Phys. Rev. D 81, 074010 (2010).

[48] Y. Aoki, T. Blum, H.-W. Lin, S. Ohta, S. Sasaki, R. Tweedie, J. Zanotti, and T. Yamazaki, Phys. Rev. D 82, 014501 (2010).

[49] H.-W. Lin, J.-W. Chen, S. D. Cohen, and X. Ji, Phys. Rev. D 91, 054510 (2015).

[50] J.-W. Chen, T. Ishikawa, L. Jin, H.-W. Lin, Y.-B. Yang, J.-H. Zhang, and Y. Zhao, Phys. Rev. D 97, 014505 (2018).

[51] R. Gupta, Y.-C. Jang, B. Yoon, H.-W. Lin, V. Cirigliano, and T. Bhattacharya, Phys. Rev. D 98, 034503 (2018).
[52] Y.-S. Liu, J.-W. Chen, L. Jin, H.-W. Lin, Y.-B. Yang, J.-H. Zhang, and Y. Zhao, arXiv:1807.06566.

[53] C. Alexandrou, K. Cichy, M. Constantinou, K. Jansen, A. Scapellato, and F. Steffens, Phys. Rev. Lett. 121, 112001 (2018).

[54] J.-H. Zhang, J.-W. Chen, L. Jin, H.-W. Lin, A. Schäfer, and Y. Zhao, Phys. Rev. D 100, 034505 (2019).

[55] R. S. Sufian, J. Karpie, C. Egerer, K. Orginos, J.-W. Qiu, and D. G. Richards, Phys. Rev. D 99, 074507 (2019).

[56] M. Oehm, C. Alexandrou, M. Constantinou, K. Jansen, G. Koutsou, B. Kostrzewa, F. Steffens, C. Urbach, and S. Zafeiropoulos, Phys. Rev. D 99, 014508 (2019).

[57] T. J. Hobbs, Phys. Rev. D 97, 054028 (2018).

[58] http://metapdf.hepforge.org/PDFSense/Lattice/.

[59] A. W. Thomas, Phys. Lett. 126B, 97 (1983).

[60] A. I. Signal, A. W. Schreiber, and A. W. Thomas, Mod. Phys. Lett. A 06, 271 (1991).

[61] A. W. Schreiber, P. J. Mulders, A. I. Signal, and A. W. Thomas, Phys. Rev. D 45, 3069 (1992).

[62] M. Alberg and G. A. Miller, Phys. Rev. Lett. 108, 172001 (2012).

[63] Y. Salamu, C.-R. Ji, W. Melnitchouk, and P. Wang, Phys. Rev. Lett. 114, 122001 (2015).

[64] P. Amaudruz et al. (New Muon Collaboration), Phys. Rev. Lett. 66, 2712 (1991).

[65] M. Arneodo et al. (New Muon Collaboration), Phys. Rev. D 50, R1 (1994).

[66] K. Gottfried, Phys. Rev. Lett. 18, 1174 (1967).

[67] G. Martinelli, C. Pittori, C. T. Sachrajda, M. Testa, A. Vladikas, Nucl. Phys. B445, 81 (1995).

[68] M. Constantinou and H. Panagopoulos, Phys. Rev. D 96, 054506 (2017).

[69] G. Apollinari, I. B. Alonso, O. Brüning, M. Lamont, and L. Rossi, CERN Report No. CERN-2015-005, FERMILABDESIGN-2015-02, 2015.

[70] S. J. Brodsky, F. Fleuret, C. Hadjidakis, and J. P. Lansberg, Phys. Rep. 522, 239 (2013).

[71] A. Accardi et al., Eur. Phys. J. A 52, 268 (2016).

[72] D. Boer et al., arXiv:1108.1713.

[73] S. Abeyratne et al., arXiv:1209.0757.

[74] E. C. Aschenauer et al., arXiv:1409.1633.

[75] J. L. A. Fernandez et al. (LHeC Study Group), J. Phys. G 39, 075001 (2012).

[76] J. Dudek et al., Eur. Phys. J. A 48, 187 (2012).

[77] A. C. Benvenuti et al. (BCDMS Collaboration), Phys. Lett. B 223, 485 (1989).

[78] A. C. Benvenuti et al. (BCDMS Collaboration), Phys. Lett. B 237, 592 (1990).

[79] M. Arneodo et al. (New Muon Collaboration), Nucl. Phys. B483, 3 (1997).

[80] J. P. Berge et al., Z. Phys. C 49, 187 (1991).

[81] U.-K. Yang et al. (CCFR/NuTeV Collaboration), Phys. Rev. Lett. 86, 2742 (2001).

[82] W. G. Seligman et al., Phys. Rev. Lett. 79, 1213 (1997).

[83] D. A. Mason, Ph. D. thesis, Oregon University, 2006, http:// lss.fnal.gov/archive/thesis/2000/fermilab-thesis-2006-01 .pdf.

[84] M. Goncharov et al. (NuTeV Collaboration), Phys. Rev. D 64, 112006 (2001). 
[85] A. Aktas et al. (H1 Collaboration), Eur. Phys. J. C 40, 349 (2005).

[86] A. Aktas et al. (H1 Collaboration), Eur. Phys. J. C 45, 23 (2006).

[87] H. Abramowicz et al. (ZEUS and H1 Collaborations), Eur. Phys. J. C 73, 2311 (2013).

[88] H. Abramowicz et al. (ZEUS and H1 Collaborations), Eur. Phys. J. C 75, 580 (2015).

[89] F. D. Aaron et al. (H1 Collaboration), Eur. Phys. J. C 71, 1579 (2011).

[90] G. Moreno et al., Phys. Rev. D 43, 2815 (1991).

[91] R. S. Towell et al. (NuSea Collaboration), Phys. Rev. D 64, 052002 (2001).

[92] J. C. Webb et al. (NuSea Collaboration), arXiv:hep-ex/ 0302019.

[93] F. Abe et al. (CDF Collaboration), Phys. Rev. Lett. 77, 2616 (1996).

[94] D. Acosta et al. (CDF Collaboration), Phys. Rev. D 71, 051104 (2005).

[95] V. M. Abazov et al. (D0 Collaboration), Phys. Rev. D 77, 011106 (2008).

[96] R. Aaij et al. (LHCb Collaboration), J. High Energy Phys. 06 (2012) 058.

[97] V. M. Abazov et al. (D0 Collaboration), Phys. Lett. B 658, 112 (2008).

[98] T. A. Aaltonen et al. (CDF Collaboration), Phys. Lett. B 692, 232 (2010).

[99] S. Chatrchyan et al. (CMS Collaboration), Phys. Rev. D 90, 032004 (2014).

[100] S. Chatrchyan et al. (CMS Collaboration), Phys. Rev. Lett. 109, 111806 (2012).

[101] G. Aad et al. (ATLAS Collaboration), Phys. Rev. D 85, 072004 (2012).
[102] V. M. Abazov et al. (D0 Collaboration), Phys. Rev. D 91, 032007 (2015); 91, 079901(E) (2015).

[103] T. Aaltonen et al. (CDF Collaboration), Phys. Rev. D 78, 052006 (2008); 79, 119902(E) (2009).

[104] V. M. Abazov et al. (D0 Collaboration), Phys. Rev. Lett. 101, 062001 (2008).

[105] G. Aad et al. (ATLAS Collaboration), Phys. Rev. D 86, 014022 (2012).

[106] S. Chatrchyan et al. (CMS Collaboration), Phys. Rev. D 87, 112002 (2013); 87, 119902(E) (2013).

[107] R. Aaij et al. (LHCb Collaboration), J. High Energy Phys. 08 (2015) 039.

[108] R. Aaij et al. (LHCb Collaboration), J. High Energy Phys. 05 (2015) 109.

[109] G. Aad et al. (ATLAS Collaboration), J. High Energy Phys. 09 (2014) 145.

[110] V. Khachatryan et al. (CMS Collaboration), Eur. Phys. J. C 76, 469 (2016).

[111] R. Aaij et al. (LHCb Collaboration), J. High Energy Phys. 01 (2016) 155.

[112] G. Aad et al. (ATLAS Collaboration), J. High Energy Phys. 08 (2016) 009.

[113] G. Aad et al. (ATLAS Collaboration), Eur. Phys. J. C 76, 291 (2016).

[114] S. Chatrchyan et al. (CMS Collaboration), Phys. Rev. D 90, 072006 (2014).

[115] G. Aad et al. (ATLAS Collaboration), J. High Energy Phys. 02 (2015) 153; 09 (2015) 141(E).

[116] V. Khachatryan et al. (CMS Collaboration), J. High Energy Phys. 03 (2017) 156.

[117] G. Aad et al. (ATLAS Collaboration), Eur. Phys. J. C 76, 538 (2016). 